University of Louisville

ThinkIR: The University of Louisville's Institutional Repository

8-2007

\title{
International student-athlete perception of college sport and its effect on adjustment to college.
}

Nels Popp

University of Louisville

Follow this and additional works at: https://ir.library.louisville.edu/etd

\section{Recommended Citation}

Popp, Nels, "International student-athlete perception of college sport and its effect on adjustment to college." (2007). Electronic Theses and Dissertations. Paper 1142.

https://doi.org/10.18297/etd/1142

This Doctoral Dissertation is brought to you for free and open access by ThinkIR: The University of Louisville's Institutional Repository. It has been accepted for inclusion in Electronic Theses and Dissertations by an authorized administrator of ThinkIR: The University of Louisville's Institutional Repository. This title appears here courtesy of the author, who has retained all other copyrights. For more information, please contact thinkir@louisville.edu. 


\title{
INTERNATIONAL STUDENT-ATHLETE PERCEPTION OF COLLEGE SPORT AND ITS EFFECT ON ADJUSTMENT TO COLLEGE
}

\author{
By \\ Nels Popp \\ B.S., Saint Mary’s University (Minn.), 1997 \\ M.S., University of Wisconsin-La Crosse, 2004
}

\begin{abstract}
A Dissertation
Submitted to the Faculty of the

Graduate School of the University of Louisville

In Partial Fulfillment of the Requirements

For the Degree of

Doctor of Philosophy
College of Education and Human Development University of Louisville
Louisville, Kentucky

August 2007 


\title{
INTERNATIONAL STUDENT-ATHLETE PERCEPTION OF COLLEGE SPORT AND ITS EFFECT ON ADJUSTMENT TO COLLEGE
}

\author{
By \\ Nels Popp \\ B.S., Saint Mary’s University (Minn.), 1997 \\ M.S., University of Wisconsin-La Crosse, 2004
}

A Dissertation Approved on

July 27, 2007

By the following Dissertation Committee

Mary A. Hums, Dissertation Co-Chair

T. Christopher Greenwell, Dissertation Co-Chair

Daniel F. Mahony

Simon Pack

Carolyn Rude-Parkins 


\section{DEDICATION}

This dissertation is dedicated to my loving wife, Stephanie, who has been with me every step of the way as I pursued this goal of completing my doctoral program. She has been incredibly encouraging and understanding throughout the process and she makes the rewards of completion twice as enjoyable.

I also would like to dedicate this work to my parents, Rod and Milli, who helped raise me to be both academically inquisitive and a true lover of sports. Without their guidance, I would not be where I am today. 


\section{ACKNOWLEDGEMENTS}

I would like to take this space to acknowledge all the wonderful people who have helped me complete this dissertation. First I would like to recognize my co-chairs, Mary Hums and Chris Greenwell. Dr. Hums has been a true inspiration, fostering and sharing my enthusiasm for the sport management field, but also tempering it with the direction of an accomplished writer and researcher. Discussions with her always left me feeling excited and encouraged about what I could achieve, yet her ability to set deadlines and writing goals were the keys to this project becoming reality.

Dr. Greenwell, meanwhile, has been extremely helpful in offering suggestions, additional sources, and insight regarding the methods used in this study. His knowledge of statistics was particularly helpful, while his personality and easy-going disposition made the tedious tasks of computing statistics and re-writing drafts much more tolerable. I also would like to recognize the other members of my committee for their excellent suggestions: Dan Mahony, Simon Pack, and Carolyn Rude-Parkins.

I would also like to acknowledge all my friends and mentors along my travels through higher education, including my instructors at Saint Mary’s University, the University of Wisconsin-La Crosse, and at the University of Louisville. I would also like to thank my great friends at Viterbo and Bellarmine Universities for giving me extra insight and opportunity in the field of sport administration. Finally, I would like to 
acknowledge the tremendous encouragement and patience of my wife, Stephanie, who really offered both unyielding support and motivation throughout the process.

I offer my most sincere appreciation to all of you. 


\title{
ABSTRACT \\ INTERNATIONAL STUDENT-ATHLETE PERCEPTION OF COLLEGE SPORT AND ITS EFFECT ON ADJUSTMENT TO COLLEGE
}

\author{
Nels Popp
}

July 27, 2007

During the 2004-05 school year, over 10,000 international student-athletes competed for National Collegiate Athletic Association schools (NCAA, 2006b). Few researchers have examined how international student-athletes’ college experiences compare to domestic student-athletes. The purpose of this study was threefold: (a) to detect differences between international and domestic student-athletes in regards to adaptation to college, (b) to detect differences between international and domestic student-athletes in regards to their view of the purpose of college sports, and (c) to determine whether student-athletes' views on the purpose of college sport help predict social adjustment to college or institutional attachment.

A national sample of international and domestic student-athletes from 11 NCAA Division I institutions completed an instrument comprised of the social adjustment and institutional attachment scales from the Student Adaptation to College Questionnaire (Baker and Siryk, 1989) and the seven factor scales from a modified version of the Purpose of Sport Questionnaire developed by Duda (1989). A total of 288 studentathletes completed the instrument, 174 of whom were international student-athletes representing 49 different countries. 
Results showed international student-athletes scored significantly lower on the social adjustment and institutional attachment scales compared to domestic studentathletes. Among the seven purpose of sport factors, only the factor of competitiveness revealed a statistically significant difference between the two groups, with international student-athletes ranking the factor lower than domestic student-athletes. None of the purpose of sport factors significantly predicted social adjustment to college for either domestic or international student-athletes. Several purpose of sport factors significantly predicted institutional attachment, however. A discussion of results and their implications are outlined. 


\section{TABLE OF CONTENTS}

DEDICATION

\section{CHAPTER}

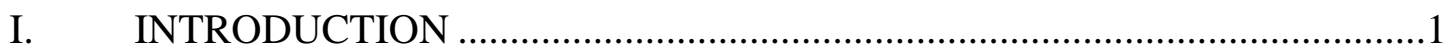

Cross Cultural Adjustment...........................................................1

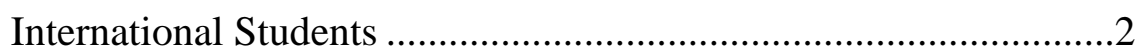

Transition from High School to College.............................................

International Student-Athlete Adjustment ..........................................

Purpose of Sport..........................................................................

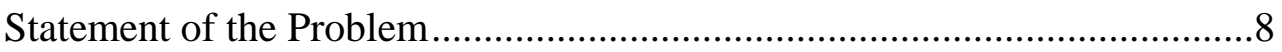

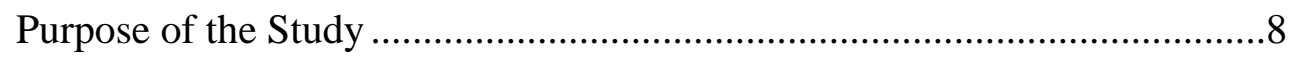

Significance of the Study ..........................................................................

Research Questions ....................................................................................10

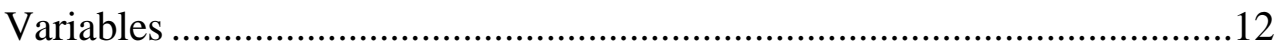

Delimitations .........................................................................................13

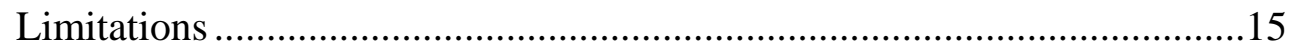



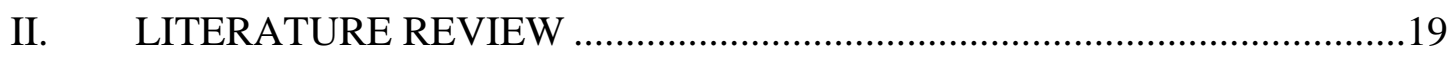

Cross-Cultural Adjustment .......................................................................20 
International Student Adjustment to College...........................................29

Student Adjustment to College .............................................................43

International Sport Policy Analysis ......................................................50

Conceptual frameworks .......................................................52

National sport policy analysis..................................................59

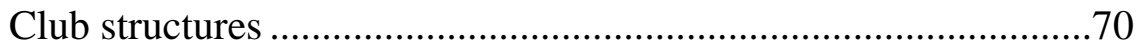

International Youth Sport Participation Trends and Youth

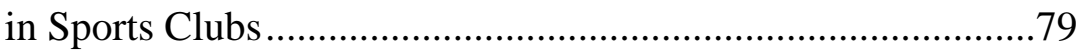

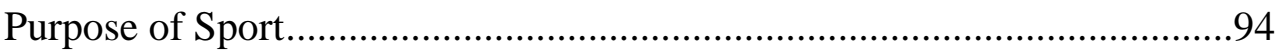

Elite Sport Transitions ...................................................................104

Student-Athlete Adjustment to College ..................................................113

International Student-Athlete Adjustment to College..............................124

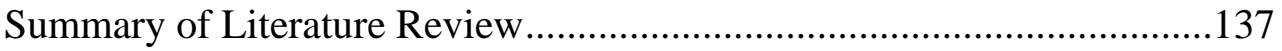

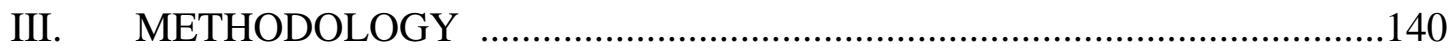

Purpose of the Study ...................................................................140

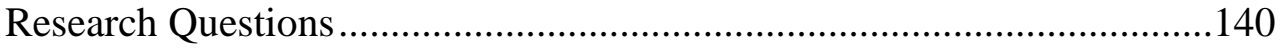

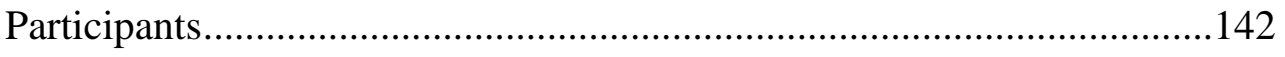



Student Adaptation to College Questionnaire............................147

Purpose of Sport Questionnaire ............................................148

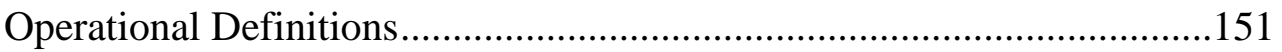

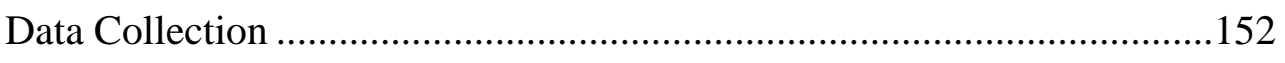

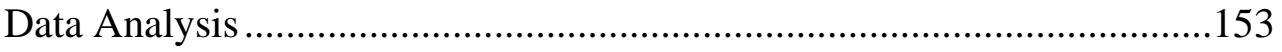


Summary of Methodology . 155

IV. RESULTS .156

Demographic Variables .156

Instrument Reliability 165



Scale Reliability and Consistency...............................................166

Mean Scores and Correlation Matrices of Instrument Factors ....169

Results of Research Questions

Assumptions of MANOVA

Differences in SACQ Factors Between International and Domestic Student-Athletes .173

Differences in Purpose of Sport Factors Between International And Domestic Student-Athletes .173

Purpose of Sport Predicting Social Adjustment and Institutional

Attachment .174

Entry of Variables Using Multiple Regression .174

Sample Size for Multiple Regression 175

Assumptions of Multiple Regression..... .175

Purpose of Sport and Social Adjustment to College .176

Purpose of Sport and Institutional Attachment .177

Summary of Results .180

V. DISCUSSION. .181

Adjustment to College .182 
Social Adjustment 183

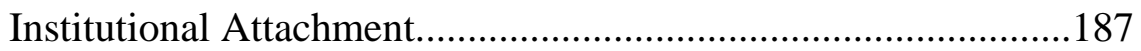

Perceived Purpose of Sport.....................................................................188

Competitiveness ........................................................................189

Mastery and Cooperation ..........................................................190

Physically Active Lifestyle ..........................................................192

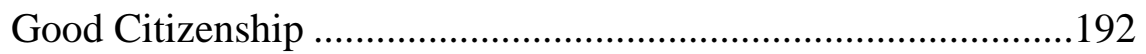

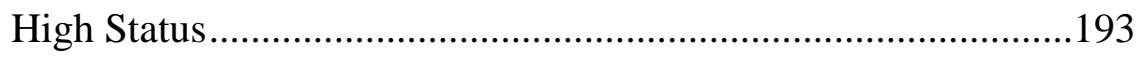

Self-esteem............................................................................194

High Social Status .........................................................................195

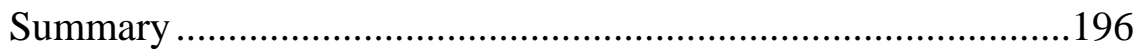

Predictive Ability of Purpose of Sport for Adjustment to College.............196

Purpose of Sport Factors and Social Adjustment ........................196

Purpose of Sport Factors and Institutional Attachment ................198

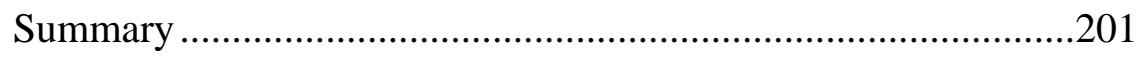

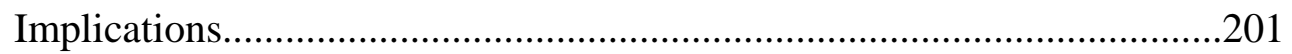

Social Adjustment and Institutional Attachment ..........................202

Purpose of Sport Factors..........................................................204

Purpose of Sport Predicting Adjustment to College .....................205

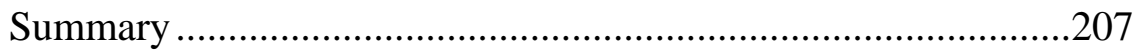

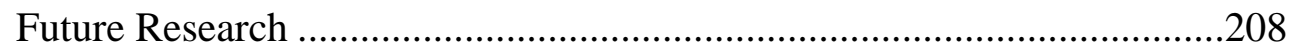

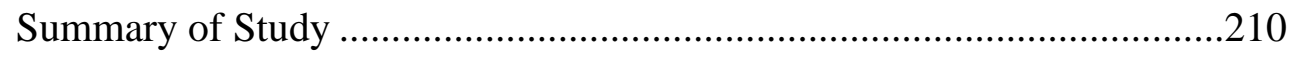

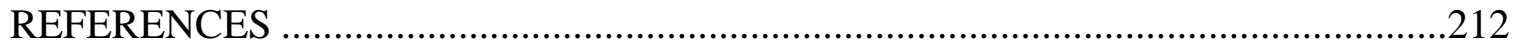


APPENDICIES .

CURRICULUM VITAE.

.231 


\section{LIST OF TABLES}

TABLE

PAGE

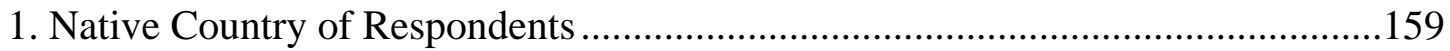

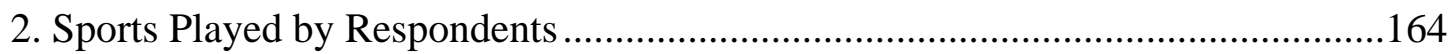

3. Inter-item Consistency Ratings of SACQ Scales ............................................168

4. Inter-item Consistency Ratings for Purpose of Sport Questionnaire Factors ........168

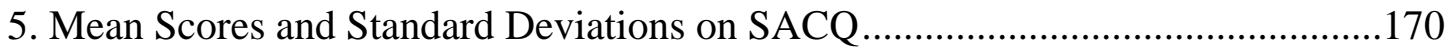

6. Mean Scores and Standard Deviations on Purpose of Sport Questionnaire ...........170



8. Correlation Matrix for Purpose of Sport Factors .................................................171

9. Multiple Regression Results for Purpose of Sport Factors Predicting Institutional

Attachment for International Student-Athletes ..........................................179

10. Multiple Regression Results for Purpose of Sport Factors Predicting Institutional

Attachment for Domestic Student-Athletes .................................................179 


\section{CHAPTER 1}

\section{INTRODUCTION}

During the 2004-05 academic year, over 10,000 student-athletes competing at National Collegiate Athletic Association (NCAA) schools were classified as non-resident aliens (NCAA, 2006b). This growing number of international student-athletes often times helped teams win conference and national titles (Brown, 2004; Litsky, 2003; Miller, 2005). Little research exists, however, examining what life on campus is like for international student-athletes and how they view their international sporting experience. International student-athletes share some traits with several frequently studied groups such as cross-cultural travelers, international students, and domestic college studentathletes. At the same time, international student-athletes share unique characteristics and circumstances which separate them from the groups mentioned above. Because of these differences, international student-athletes have their own unique needs and may view the college sports experience in a different manner than domestic student-athletes.

\section{Cross Cultural Adjustment}

Several researchers have examined the concept of cross-cultural adjustment and how it affects those who leave one culture and submerge themselves in another (Adler 1975; Church 1982). Cross-cultural travelers mentioned in the literature, including missionaries, Peace Corps volunteers, international business travelers, refugees, and international students, often endure a series of fluctuating degrees of self- and cultural- 
awareness during their transitional experience, often referred to as the U-curve or $\mathrm{W}$ curve. Initially, these travelers experience feelings of excitement upon arrival in the new culture, but after this initial feeling wears off, they may enter a period of confusion and disorientation regarding the new culture. Quite often this stage is followed by a rejection of the new culture and a longing for the native culture, including its familiar reinforcements of behavior. As sojourners begin to understand and accept the new culture, however, they adapt a heightened sense of awareness and sensitivity to their new environment and of themselves. Independence marks the final stage in which the traveler fully accepts and understands the new culture and its relationship to personal beliefs and values (Adler, 1975; Lysgaard, 1955). Because international student-athletes often spend several years submerged in a foreign culture, it is important to understand what feelings and psychological stresses they may be enduring during this time away from their native homeland.

\section{International Students}

One group of cross-cultural sojourners receiving considerable scholarly attention is international students. Some of the studies regarding cross-cultural sojourners have addressed concerns and feelings of international students and what particular strains and stressors they endure during the adjustment process (Constantine, Anderson, Caldwell, Berkel, \& Utsey, 2005; Crano \& Crano, 1993; Parr, Bradley, \& Revathi 1992). Important issues for international students included: (a) socialization opportunities, (b) cultural differences, (c) staying connected to family, (d) the financial burden of international college attendance, (e) an attachment to their United States college, and (f) an understanding of how classes are taught in United States colleges. Other researchers have 
investigated the importance of native country as a factor for international student adjustment (Chapdelaine \& Alextich, 2004), and the importance of social networks for successful college adjustment of international students (Rajapaksa \& Dundes, 2002).

\section{Transition from High School to College}

The literature also contains several studies regarding the adjustments made by domestic students when they transition from high school to college. Baker and Siryk $(1984,1986,1989)$ in particular have been at the forefront of college student adjustment research, and have developed a commonly used instrument called the Student Adjustment to College Questionnaire (SACQ; 1989) which has been used extensively to evaluate how students adapt to college. Their instrument is based on the concept of student adjustment as a multi-faceted construct. The SACQ is based on four facets, or adjustment factors, which are used in many college adjustment studies. They are: (a) academic adjustment, (b) personal-emotional adjustment, (c) social adjustment, and (d) institutional attachment. A fifth, full-scale adjustment score is typically determined and reported as well. In addition, several researchers have conducted studies on the adjustments made by domestic student-athletes who transition from high school to college. The adjustment process for these student-athletes is often times not the same as it is for non-studentathletes (Adler \& Adler, 1985; Howard-Hamilton \& Sina, 2001; Pascarella, Truckenmiller, Nora, Terenzini, Edison, \& Hagedorn, 1999). Domestic student-athletes have been shown to hold different perceptions about academic expectations and in some studies have demonstrated lower levels of academic achievement compared to their nonathlete classmates. 


\section{International Student-Athlete Adjustment}

Despite the considerable attention paid to international student adjustment and domestic student-athlete adjustment, few researchers have looked at the adjustments of international student-athletes. Evidence does suggest the adjustment process for international student-athletes differs from that of other international students (Bale, 1991; Ridinger, 1998). Unlike many international students, international student-athletes often enter college in the United States as part of a pre-formed socialization network comprised of teammates and other athletes. Also, international student-athletes often receive athletic scholarships, greatly reducing the financial burden of college attendance which often affects international students.

Evidence also suggests international student-athletes adjust to the college experience differently than do domestic student-athletes. International student-athletes are often better prepared academically for the rigors of college study (Bale, 1991; Ridinger, 1998; Ridinger \& Pastore, 2000a). International student-athletes often come from a club-based sport system, which is quite different than the school-based sport system they find in the United States (Chalip, Johnson, \& Stachure, 1996; Rubingh \& Broeke, 1998). This difference in sport systems requires an adjustment period. Many international student-athletes have never participated under a system in which their eligibility to play sport is linked to their academic success or their failure to accept past financial remuneration to play in sport competitions. These are, however, the parameters of participation in NCAA athletics. Some researchers have also suggested international student-athletes often must adjust to the high athletic demands and emphasis placed on 
student-athletes at NCAA schools (Bale, 1991; Popp, 2006) despite the sport participation taking place in institutions of higher learning.

A handful of researchers have examined different elements of the international student-athlete experience. Bale (1987) investigated how international student-athletes make migration decisions and how they feel about those decisions. He also documented the history of college recruitment of international athletes and how they reacted to their experiences in the United States (Bale, 1991). Several key variables have changed since his work, including more NCAA legislation regarding international student-athletes; a more competitive recruiting environment; more knowledgeable coaches, scouts, and athletes; and more accessible information regarding schools and opportunities. Bale also noted United States coaches now recruit in more locations around the world, such as Eastern Europe, which were largely untapped during his research (J. Bale, personal communication, November 12, 2005).

Ridinger (1998) compared antecedents and outcomes of adjustments to college made by various sub-groups at a single university, including domestic and international student-athletes. Using Baker and Siryk’s (1989) Student Adaptation to College Questionnaire (SACQ), she found international student-athletes scored higher on adjustment scales than the other groups of domestic student-athletes, international students, and even domestic students. Her exploratory study using a sample size of 16 international student-athletes all from a single institution, however, was not large enough to address certain issues of generalizability and validity to the total international studentathlete population. 
Other researchers have demonstrated a need to examine differences in how athletes perceive changes in their sporting experience. Athletes must make certain adjustments when they advance from a lower level to a higher level, a process called ‘athlete transition’ (Green, 2005). Little empirical research has documented adjustments made by athletes during this transition. Chambliss (1989), in his ethnographic study of elite United States swimmers, suggested a qualitative difference, but not a quantitative one, between competitive and high performance athletes. International student-athletes moving from competition in their homeland to elite NCAA Division I competition in the United States would likely experience such athlete transitions.

Other reasons exist supporting the notion that international student-athletes will have a different perception regarding the purpose of sport compared to their United States-born teammates. Research on international sport policy has shown sport participation opportunities are different from one nation to the next (Chalip, Johnson, \& Stachure, 1996; Green, 2005; Palm, 1991; Stahl, Rutten, Nutbeam, \& Kannas, 2002) and different levels of sporting opportunity are emphasized quite differently depending on the politics, history, and ideology of that nation and its policy makers (Chalip, 1995; Green \& Oakley, 2001; Harvey, Beamish, \& Defrance, 1993). Also, many international studentathletes develop their athletic abilities in the club system, which is quite different from the school-based system used in the United States (Rubingh \& Broeke, 1998). Several authors have called for further examination into the differences in cross-cultural sport development (Chalip et al., 1996; Duda \& Allison, 1990; Green \& Oakley, 2001), and, more specifically, adjustment differences between international and domestic studentathletes (Ridinger \& Pastore, 2000a; 2000b), but the literature does not address such 
issues. Stidwill (1984) looked at differences in athletic motivation between domestic and international college track and field athletes, and found a significant difference in perceived athletic confidence, with international student-athletes demonstrating higher levels of confidence in their ability to achieve athletic success, which would suggest differences in how international student-athletes perceive college competition compared to domestic student-athletes might also exist.

Purpose of Sport

If international student-athletes do indeed perceive the purpose of collegiate sport differently than domestic student-athletes, finding an instrument to measure such differences is critical. A handful of studies have examined differences in participants' perceptions of the purpose of sport, although this study is the first to do so using a crosscultural sample. To measure perceptions of the purpose of sport, Duda (1989) developed an instrument to use with United States high school students. Results from the use of her instrument, called the Purpose of Sport Questionnaire, revealed seven different factors regarding what sport should do for participants as demonstrated by the following factor headings developed by the author: (a) mastery/cooperation, (b) physically active lifestyle, (c) good citizen, (d) competitiveness, (e) high-status career, (f) enhance self-esteem, and (g) social status/getting ahead.

This Purpose of Sport Questionnaire has since been used in several other studies to detect differences in participants' perceptions of the purpose of sport. White (1995) used the instrument with college students to detect differences between varsity and recreational sport participants. Other studies used the instrument to detect differences in perception of purpose of sport between amateur and professional English rugby players 
(Treasure, Carpenter, \& Power, 2000) and between amateur and professional English soccer players (Carpenter \& Yates, 1997). While the Purpose of Sport Questionnaire has not been used with cross-cultural sport participants, Duda and Allison (1990) suggested more research was needed to fill the void in the field of cross-cultural sport psychology.

\section{Statement of the Problem}

International student-athletes face unique circumstances as they adapt and adjust to life on their United States college campus. Little is understood, though, about how well international student-athletes adjust to college and what factors affect their ability to adjust. International student-athletes come from backgrounds which are quite different from college student-athletes who grow up in the United States. These various sporting and cultural backgrounds are likely to produce different perceptions regarding the purpose of sport and could affect the international student-athlete's ability to adjust to life at various United States universities and colleges.

\section{Purpose of the Study}

This study has several purposes. The first purpose of this study is to assess whether international student-athletes demonstrate different abilities to adjust to college as compared to domestic student-athletes. Next, the study examines whether international student-athletes view the purpose of collegiate sport differently than student-athletes from the United States. Third, this study examines whether perception of the purposes of collegiate sport can predict both domestic and international student-athletes’ ability to adjust to college. 


\section{Significance of the Study}

Because the number of international student-athletes competing at the NCAA level continues to increase, it is important to understand how international studentathletes' experiences affect their well-being. Despite the media attention paid to schools and coaches, student-athletes remain the primary stake-holders in NCAA competition. Their experiences are critical when assessing the value of the NCAA and the competitions and institutions it oversees. A portion of the NCAA's core purpose is "to integrate intercollegiate athletics into higher education so that the educational experience of the student-athlete is paramount” (NCAA Executive Committee, 2004). Such a statement calls for study into the well-being of all NCAA student-athletes, including those from other nations. Few studies, though, have examined the experience of international student-athletes. Past research has revealed several factors which make college adjustment more difficult for certain groups of students. These factors include: (a) the jump from high school to college, (b) attending college in a foreign country, and (c) the high demands placed on NCAA Division I college athletes. International studentathletes must make adjustments to meet all these challenges. Little is known, however, about how these challenges impact international student-athletes physically, socially, psychologically, or academically.

College students who do not adapt or adjust well to college, particularly in the areas of social adjustment and institutional attachment, are more likely to have trouble being away from home and are more likely to leave school before graduation (Baker \& Siryk, 1989). International students have been shown to be particularly susceptible to poor adjustment in these two areas (Kaczmarek, Matlock, Merta, Ames, and Ross, 1994). 
College coaches, administrators, teammates, and even spectators are affected by the ability of international student-athletes to adjust to college. International student-athletes who do not adjust well to college are more likely to leave, which can hurt team cohesion and affect a team's academic progress report (APR) scores, meaning the possible loss of scholarships. The loss of international student-athletes who are not socially well adjusted can also negate any competitive advantage gained by landing elite student-athletes from other countries. International student-athletes, particularly in sports such as tennis, soccer, swimming, and basketball, give some lower profile universities the opportunity to compete with schools attracting elite United States-born student-athletes.

Results of this study could assist coaches and administrators in making the college athletics experience a more positive and productive one for international student-athletes. The positive experience of an international student-athlete could lead to increased recruitment of top international athletes, which would improve team performances and perhaps even spectator attendance. International student-athletes who have positive experiences in their United States colleges could also improve educational opportunities for domestic student-athletes--and students--who are exposed to other cultures through the international student-athlete. Many universities espouse the values of a global education. Offering positive international experiences to student-athletes from other countries can assist schools in meeting this mission.

\section{Research Questions}

This study contains three primary research questions: (a) Do international studentathletes adjust better to college than domestic student-athletes?, (b) Do international student-athletes view the purpose of collegiate sport differently than domestic student- 
athletes?, and (c) Do student-athletes' perspectives of the purpose of collegiate sport affect their adjustment to college? In order to answer these three primary questions, additional sub-questions are posed for each primary question. The first primary research question is divided into two sub-questions, the second primary research questions is divided into seven sub-questions, and the third primary questions in divided into two subquestions. Those sub-questions are listed below:

1a. Are there significant differences between international student-athletes and domestic student-athletes in their ability to socially adjust to college?

1b. Are there significant differences between international student-athletes and domestic student-athletes in their institutional attachment to their United States college?

2a. Are there significant differences between international student-athletes and domestic student-athletes in their view of the purpose of sport as a means of mastery and cooperation of athletic skill?

2b. Are there significant differences between international student-athletes and domestic student-athletes in their view of the purpose of sport as leading to a physically active lifestyle?

2c. Are there significant differences between international student-athletes and domestic student-athletes in their view of the purpose of sport as helping an individual become a good citizen?

2d. Are there significant differences between international student-athletes and domestic student-athletes in their view of the purpose of sport as making an individual more competitive? 
2e. Are there significant differences between international student-athletes and domestic student-athletes in their view of the purpose of sport as leading to a high status career?

2f. Are there significant differences between international student-athletes and domestic student-athletes in their view of the purpose of sport as enhancing selfesteem?

2g. Are there significant differences between international student-athletes and domestic student-athletes in their view of the purpose of sport elevating social status and helping individuals get ahead?

3a. Do purpose of sport factors help predict social adjustment for either international or domestic student-athletes?

3b. Do purpose of sport factors help predict institutional attachment for either international or domestic student-athletes?

\section{Variables}

The demographic variables in this study included: (a) gender, (b) year in college, (c) native country, (d) years spent in the United States, (e) approximate cumulative grade point average, and (f) varsity sport played in college. The independent variable of interest for most of the research questions was whether a student-athlete was international or domestic. Such status was derived from responses to the demographic item "native country.” If student-athletes listed any country other than the United States in response to this item, they were considered an international student-athlete in this study, while all student-athletes listing the United States as their native country were considered domestic student-athletes. 
This independent variable of student-athlete status was analyzed in regard to different sets of dependent variables. The first set included adjustment to college scores derived from the Student Adjustment to College Questionnaire (SACQ; Baker and Siryk, 1989), which is explained further in Chapter 3 . These scores measured two factors of the SACQ: social adjustment, and institutional attachment. The second set of dependent variables came from the Purpose of sport Questionnaire (Duda, 1989), which measured seven factors: (a) mastery/cooperation, (b) physically active lifestyle, (c) good citizen, (d) competitiveness, (e) high status career, (f) enhance self-esteem, and (g) social status/getting ahead.

This study also examined whether student-athletes' perception of the purpose of sport predicted their social adjustment or institutional attachment to college. In these analyses, the seven purpose of sport factor scores served as independent variables, while the two adjustment to college factor scores served as dependent variables. Two multiple regression analyses were run for both domestic and international student-athletes.

\section{Delimitations}

This study was designed to capture data from international and domestic studentathletes attending college at NCAA Division I institutions nation-wide. The decision to collect data from schools nationally was made for two reasons. The first reason was no single institution has a large enough international student-athlete population to create an appropriate sample size. The only previous quantitative study to examine international student-athlete adjustment to college (Ridinger, 1998) utilized data from a single school, resulting in data collection from just 16 international student-athletes, limiting the generalizability and validity of the study results. A second reason for collecting data 
nationally stems from prior research, which suggested international student-athletes attend universities located in different areas of the country at disproportionate rates (Bale, 1987). A national sample was necessary to collect data from international student-athletes at both rural and urban schools and at schools with both small and large enrollments.

The decision to only include Division I student-athletes in the study was made for three reasons. The first reason was collecting data from participants attending several universities across the country required assistance. After discussing collection methods with other sport administration faculty and athletics department administrators, a decision was made to work through the coordinators of a program called CHAMPS/Life skills. The CHAMPS/Life Skills program was initiated by the NCAA in 1991. Schools participating in the initiative develop programs offering support and services to assist student-athletes. International student-athletes are one of the primary targets for CHAMPS/Life Skills programs. Of 466 CHAMPS/Life Skills programs in place at NCAA schools in 2004, 300 of them were located at Division I institutions (NCAA, 2004). In addition to having more programs, larger Division I schools were more likely to have staff members whose primary responsibility was the CHAMPS/Life Skills program. Division II and Division III schools were more likely to have programs administered by staff also having other responsibilities within the department.

The other reason for using a sample of strictly Division I student-athletes was simply because the largest percentage of international student-athletes attend Division I institutions. During the 2004-05 academic year, nearly 70\% of the international studentathletes participating in NCAA competition were doing so at the Division I level (NCAA, 2006b). In fact, according to that NCAA report, only 2,305 student-athletes among the 
79,869 total competing at NCAA Division II schools were non-resident aliens (2.9\%) and only 1,050 student-athletes among the 151,059 NCAA Division III total were nonresident aliens (0.7\%). Division I schools offered the best chance of collecting data from greater numbers of participants attending fewer institutions. In addition, using only Division I student-athletes, as opposed to all divisions, allowed for a more homogeneous population, which reduced error when examining the data.

\section{Limitations}

One limitation of the current study is the dependence on assistance from CHAMPS/Life Skills coordinators. Requests for assistance in data collection were made by phone to CHAMPS/Life Skills coordinators. Only student-athletes who attended schools from coordinators who verbally agreed to help were included in the sample. Findings should be generalizable to the entire international student-athlete population because of the number of participants involved, but certain schools may attract international student-athletes who vary in traits and characteristics from those involved in this study. In addition, student-athletes attending school without CHAMPS/Life Skills coordinators may be less well adjusted to college because assisting students with adjustment to college is one of the primary functions of CHAMPS/Life Skills coordinators.

Another limitation of this study is the use of modified instruments. The SACQ has been used in numerous studies on college adjustment, consistently meeting high levels of validity and reliability. The instrument always produces five scores including four factor scores and one full-scale score. The full SACQ, however, contains 67 items. 
The second instrument used in this study was a modified Purpose of Sport Questionnaire (Duda, 1989). This questionnaire originally had participants respond to items following a stem that read "A very important thing sport should do is..." Other studies have altered this stem to read "A very important thing that soccer should do is..." (Carpenter \& Yates, 1997) and “A very important thing rugby should do is...” (Treasure, Carpenter, \& Power, 2000). All previous studies using this questionnaire have produced high levels of validity and reliability, despite stems varying slightly. A decision was made for this study to have the stem read "A very import thing college sport should do is:”. The original Purpose of Sport Questionnaire contained 46 items.

It was believed an instrument comprised of 113 total items (67 items from the SACQ and 46 items from the Purpose of Sport Questionnaire), plus demographic variable questions, would not achieve a high response rate among college student-athletes. As Dillman (2000) pointed out, longer questionnaires generally produce lower response rates. Because of this, decisions were made to eliminate some items from the instrument, or in the case of the SACQ, eliminate some factors altogether. In the SACQ, only the factors of social adjustment and institutional attachment were used in this study. Previous researchers have successfully extracted one factor from the SACQ to measure a specific adjustment trait (Hannum \& Dvorak, 2004). In addition, certain low-loading and less relevant items on the Purpose of Sport Questionnaire were eliminated reducing the instrument to a more manageable level of items, which Dillman (2000) suggested would improve response rate. Such a reduction of items, however, limits the type of data collected for this study. 
A third limitation of this study was the categorization of all international studentathletes into a single variable. International student-athletes come from numerous countries and previous research suggests differences in adjustment and perception can be attributed, at least in part, to country of origin (Chapdelaine \& Alextich, 2004). International student-athletes could very well have quite different experiences from one another, yet in this study, international student-athletes are categorized as one sub-group. Using individual countries as independent variables would not render enough participants to run appropriate statistical analysis of the data. So despite the fact student-athletes from Kenya may have quite different experiences in the United States than student-athletes from Israel or Norway, such differences were not uncovered in this study. Due to the lack of research on international student-athletes, though, it is necessary to start somewhere. Significant differences detected in this study between international and domestic studentathletes may lead to future studies which examine differences between international student-athletes from particular geographical regions or countries.

A fourth limitation of this study is the number of demographic variables assessed. The review of literature suggested international students who attend different sized schools, in different geographic regions, in different sized communities, or stay for different lengths of time in the United States may demonstrate varying levels of adjustment (Bale, 1987; 1991; Craven 1994). Other pertinent factors include sport played, previous international travel experience, participation opportunities in home country, and gender. All these factors could play a role in how international student-athletes adjust to college and how they perceive the sport experience. To explore all these variables, though, a very large sample size would be necessary, and is beyond the scope of the 
current study. This limitation also demonstrates the real dearth of research in this particular field of study.

\section{Definitions}

Club-based sport system - The structure in place in countries where most organized sport opportunities are administered by voluntary clubs in which the focus of the clubs is on members, volunteers, and self-organization (Rubingh \& Broeke, 1998).

Domestic Student-Athletes - A university student who is a citizen of the United States and is also a member of an intercollegiate varsity athletic team.

Institutional Attachment to College - A university “student’s degree of commitment to educational goals and degree of attachment to the particular institution the student is attending, especially the quality of the relationship or bond that is established between the student and the institution.” (Baker \& Siryk, 1989, p. 15).

International Student-Athletes - A university student who is a citizen of a country other than the United States and is also a member of an intercollegiate varsity athletic team.

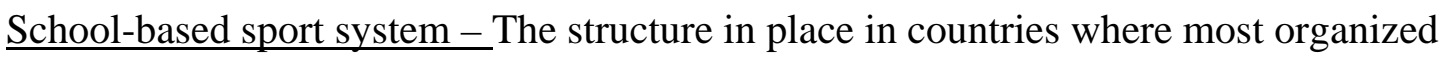
sport opportunities are administered through the educational system such as college sport and high school sport (Rubingh \& Broeke, 1998).

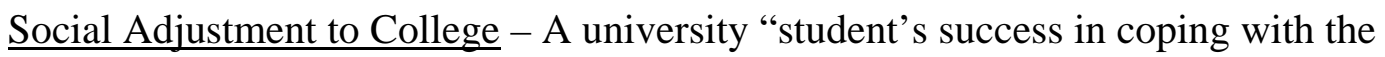
interpersonal-societal demands inherent in the college experience” (Baker \& Siryk, 1989, p. 15).

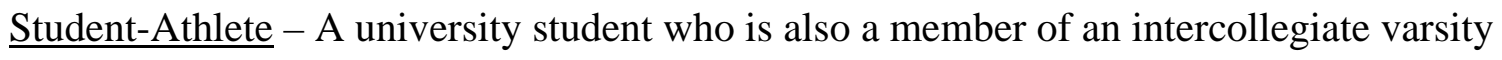
athletic team. 


\section{CHAPTER 2}

\section{LITERATURE REVIEW}

Despite the presence of over 10,000 international student-athletes on United States university campuses (NCAA, 2006b), little research exists examining the adjustments made by such cross-cultural sojourners. International student-athletes develop their interest and ability in sport in their home nations, but travel to the United States where they participate in elite university competition through structures such as the National Collegiate Athletic Association (NCAA) or the National Association of Intercollegiate Athletics (NAIA). Because different nations have unique sport policies and educational systems, international student-athletes must make adjustments in order to fully participate in their new culture. The following literature review summarizes relevant research in many areas pertinent to the study of the international student-athlete experience.

The literature review begins with a synopsis of cross-culture adjustment research, starting with general cross-cultural adjustment, then covers more specific research regarding international student adjustment to college and domestic student adjustment to college. Following the review of cross-cultural and college adjustment literature is a summary of studies examining international sport policies, which enables readers to understand why various nations employ different policies to govern sport. The sport 
policy section also reviews research on club-based sport policy, which exists in most nations outside of North America.

The next topic covered in the literature review is a section entitled "purpose of sport.” Articles reviewed in this section cover research examining how different sets of athletes, such as high school students, college students, amateur athletes, and professional athletes view the purpose of sport participation. Following the review of "purpose of sport" literature is a section examining elite sport transition, which covers research on the adjustment process athletes endure when moving from one level of competition to another. The final two segments of the literature review discusses research depicting adjustments made by college student-athletes and international student-athletes.

\section{Cross-cultural Adjustment}

The experience of spending an extended amount of time in an unfamiliar culture can be quite traumatic to individuals. While the current review of literature is mainly concerned with international student-athletes, many groups and individuals go through cross-cultural experiences. Refugees, international business travelers, missionaries, military personnel, and exchange students are just some of the people who experience cross-cultural adjustment. Several studies examined the affects of cross-cultural sojourn, including the concept of culture shock and the U-curve theory of adjustment. These concepts are outlined and explained by several authors, starting with Adler's (1975) composite study of transitional experience.

Adler (1975) developed a model outlining the transitional experiences of people who spend time living in a culture different from their own. He began with a definition of “culture shock", a term first developed by Oberg (1958), stating it is primarily "a set of 
emotional reactions to the loss of perceptual reinforcements from one's own culture, to new cultural stimuli which have little or no meaning, and to the misunderstanding of new and diverse experiences" (p. 13). He argued culture shock can be a form of alienation, but also a method leading to increased awareness and an ability to cope with a new culture.

His model began with four premises: (a) the human experience is a combination of influences from other groups and of self meaning-making, (b) the idea of culture is a "pattern of perceptions expected by an identity group” (Adler, 1975, p. 14), (c) most people do not really know their own beliefs, values, and opinions until they are tested, and (d) new experiences create what he labels "personality disintegration" in which an individual's personality moves into a higher level of awareness after experiencing change or conflict in environment. Adler suggested his model of the transitional experience helps explain the feelings and psychological changes one encounters when submerged into a new culture.

Individuals going through transitional experiences suddenly develop a keen sense of their cultural awareness, something seldom found in those who do not leave their cultural environment. This cultural awareness comes about through a series of stages, often referred to as the U-curve or W-curve. During the first stage, called contact, the sojourner feels excitement to be in a new culture. The disintegration stage follows the contact stage, in which the sojourner becomes much more aware of the differences in culture and feels isolated or alienated. The third stage, which Adler labels reintegration, is marked by a rejection of the new culture and a seeking out of connections to the home culture. Individuals in this stage may feel hostility toward the foreign culture which can result in a façade of acceptance but a desire to return to the home culture. Autonomy is the 
fourth stage, in which an individual begins to truly understand and appreciate the new culture. The final stage is independence, where the sojourner can fully accept and enjoy the new culture, but is also aware of other cultural influences and understands each person, including self, is a product of his or her environment.

As one moves through this transitional experience, the individual becomes less dependent on cultural reinforcements of behavior and more understanding of how different cultures shape personality. Adler (1975) suggested his model could be used to help sojourners be more prepared for culture shock and offered readers a better understanding of their own cultural values and beliefs.

Church (1982) put together an important critical review of research available regarding psychological adjustments made by international sojourners. His work linked several lines of study, giving readers a strong scope of the field at the time of publication. The research he examined dealt primarily with international students, but also considered several studies involving overseas missionaries, members of the Peace Corps, international business travelers, and other groups. All studies investigated adjustments mid- to long-term visitors faced in their host country, which Church labeled "sojourner adjustment.”

Church (1982) cited Oberg (1960) as the first researcher to use the term "culture shock”, calling it an "occupational disease” affecting people who are suddenly submerged into a new culture. Effects of culture shock include anxiety, helplessness, irritability, and a longing for a more predictable environment. Most research in the field since Oberg's initial work has focused on different variables associated with sojourner adjustment. 
Church (1982) began with a summary of descriptive approaches to adjustment, including: (a) stage, (b) U-curve, (c) types and patterns, and (d) culture learning. Stage descriptions of sojourner adjustment include four stages: (a) a honeymoon period, (b) a period of hostile and stereotypical attitude towards host nationals, (c) a recovery stage, and (d) complete adjustment stage. These stages can be summarized through the U-curve model of adjustment, previously documented by several researchers. The investigator also noted, however, several additional researchers who have disagreed with the U-curve theory through studies showing contrary results. He also established a grouping of research which examined types or patterns of sojourners and their adjustment ability, stating more conservative, traditional, and conforming sojourners struggle with adjustment more than those on the other end of the spectrum. Research in the culture learning vein illustrates how the loss of positive reinforcement from traditional cultural rewards, such as food, friendship, and entertainment in a new culture can make social adjustment more difficult.

Church (1982) discussed many of the background variables researchers have examined and their various effects. Several studies observed the variable of sojourner nationality and found distinct differences between ability to adjust for citizens of one country compared to those of another. Church pointed to a study by Galtung (1965) as the only one to compare a nation's sojourners and their adjustments to multiple countries, a notable hole in the literature. Other background variables investigated included personal status in home country compared to host country and previous cross-cultural experience before making the sojourn.

Church (1982) also reviewed studies looking at situational variables for the 
sojourner, as he stated the context in which the participant finds himself or herself is of major significance. Variables in this field included sojourners who were students, business personnel, or members of the Peace Corps, all of whom had distinctively different reasons for being in another country. Connected to these variables were the nature and extent of the sojourner's journey, which Church mentioned as another line of research.

Church (1982) noted another line of research in sojourn adjustment, recognizing the nature and extent of social encounters made by sojourners with host nationals. These relationships often defined sojourners ability to adjust well. Some sojourners kept to themselves in the foreign country, or intentionally remained in groups surrounded by conationals. Some research has found sojourners desire contact and relationships with host nationals, but are afraid to seek it out. A difficulty in this examination, though, is measuring types of relationships. Another issue related to this field is overlapping membership conflict, in which sojourners try too hard to fit in and lose the connection with their native culture. Personality variables also play a strong role in successful or unsuccessful sojourn adjustment and have been the topics of several studies.

Finally, Church (1982) outlined several important criticisms in the field of sojourner adjustment. These include overgeneralization because of limited sample size, heavy reliance on survey instruments which do not measure enough critical variables, and a lack of longitudinal studies to follow the process of sojourner adjustment, among others.

Befus (1988) developed and tested a multilevel treatment for travelers to other countries, helping them to combat culture shock, or cross-cultural adjustment, as it is also 
called. The study examined $96(N=96)$ North American students studying Spanish at a university in Costa Rica. She split the group into two cohorts, with each group beginning their language study at a different time so members from the control group would not affect members of the experimental group. The control group contained 64 participants, while the experimental group totaled 32 participants. The researcher utilized correlated samples t-tests to compare results.

Befus (1988) asserted only a multilevel treatment can help alleviate the pitfalls of culture shock. The researcher also listed several sojourner outcomes of culture shock, which included psychological and physical trauma. The purpose of this study was to find methods to alleviate such negative outcomes.

In her study, Befus (1988) used a psychological distress instrument called the SCL-90-R, comprised of questions measuring levels of discomfort on a 5-point Likerttype scale with anchors of 0 (“not at all”) to 4 (“extremely”). This survey measured levels of stress and the scores from the survey were the dependent variables in the research project. The survey also contained questions regarding basic demographic information such as sex, marital status, level of education, age, religious affiliation, previous crossculture experience, and amount of exposure to subcultures within North America.

The first cohort, the control group, began their foreign language studies in the Costa Rican school in September, 1984. After three months, the subjects took the SCL90-R. The experimental group began their studies in January, 1985, but also had the option of attending one-hour courses twice a week to help counter culture shock. Fiftytwo subjects volunteered to take the courses but only 32 (the sample population) attended seven or more of a possible 12 classes. Cohort group served as the independent variable. 
After running the t-test, Befus (1988) found a $t$ value of 2.615, which was significant ( $<$ $.01)$.

The researcher did point out several shortcomings of her study. The population sample was quite homogenous, as all participants were Caucasian and from North America, and nearly all were training to be Protestant missionaries, so they held similar belief systems. Befus (1988) also noted the study would be difficult to replicate because the courses in combating culture shock could only be taught by trained psychologists. Finally, questions could be raised about the use of two separate cohorts instead of random sampling of a single cohort.

Phalet and Hagendoorn (1996) examined adjustment factors affecting Turkish youth who must acculturate to different environments. The researchers looked at two different aspects of personal adjustment: internal and external. They also differentiated values of collectivism versus achievement in the acculturating participants. Internal adjustment refers to personal factors such as wellbeing, satisfaction, and health, while external adjustment referred to acquisition of the social and instrumental skills necessary for everyday activity in a new environment. Collectivistic values were defined as those of conformity, security, and tradition, while individualistic values were described as autonomy, hedonism, and stimulation. The youth in their study came from two different settings, those who had moved from rural Turkey to urban Istanbul and those whose families had moved from Turkey to Belgium. The youth who had moved from a rural to urban environment were stratified into three socio-economic status (SES) levels while the Turkish youth in Belgium were all classified as low-SES because their fathers primarily worked as low-wage miners. 
The researchers set three hypotheses for their study, based on the literature. They believed acculturating participants who had a collectivistic orientation would have an easier time adjusting both internally and externally. They also suggested the larger the gap in social equality (SES) or cultural distance (remaining in their native country compared to moving to Belgium), the more adjustment problems would surface. Their third hypothesis posited greater gaps in social equality or cultural distance would generate a more collectivistic nature in the acculturating group.

Phalet and Hagendoorn (1996) used causal models to test their hypotheses. They selected a sample of 309 Turkish youth in Istanbul who had migrated from rural regions and a sample of 100 native Turkish youth who had moved with their families to Belgium. The Istanbul sample had 141 girls and 168 boys, and had 82 participants in the low-SES group, 117 in the middle-SES group, and 110 in the high-SES group. Other demographic information was also collected. Participants then completed Nuttin’s (1980) Inventory of Motivational Objects (INOM), which assessed collectivism and individualistic values. The researchers measured external adjustment by presenting participants with four simulated tasks and having them solve the tasks by either using problem-solving behaviors or avoidance behaviors. The 20-item Cornell Medical Index (Brodman, Erdmann, Lorge, Gerhenson, \& Wolff, 1952) provided an instrument for assessing internal adjustment.

Results of the stepwise causal model demonstrated a significant positive correlation between collectivism and both internal and external adjustment. In the Istanbul sample, different SES levels negatively correlated with the amount of internal adjustment problems but not external adjustment problems. The researchers did suggest 
low-SES rating did lead to increased collectivism values and said girls associated more with collectivistic values and felt more stress than boys in the same SES level.

A second causal model examined differences between the Istanbul sample and the Belgium sample. Participants with high collectivism values were more effective in daily activity, thus lowering stress scores. Scores did demonstrate cultural distance negatively affected external adjustment scores, but surprisingly, cultural distance positively affected internal adjustment in the sample. The Belgium sample, which consisted of all low-SES scores, scored significantly higher on collectivistic values than did low-SES participants in the Istanbul sample.

Phalet and Hagendoorn's (1996) study showed cross-cultural groups which associate with their own national peers have higher collectivistic values and adjust better externally, but have more stressful internal adjustments. In other words, they use group association or identification with others of their same nationality to become more comfortable in their new environment, which comes at the expense of learning and acculturating to their new host environment.

As outlined in the studies listed above, individuals who relocate from one culture to another for an extended period of time often go through many adjustments. International student-athletes are no exception. Those who have studied cross-cultural adjustment point out many factors effecting how slowly or quickly cross-cultural adjustment occurs. Most researchers are in agreement regarding the pattern of adjustment. They also emphasize pre-sojourn factors and relationships during the transition can greatly impact ability to adjust, an important point when analyzing international student-athlete adjustment. 


\section{International Student Adjustment to College}

Numerous studies deal with the adjustment factors associated with international students. International students manage many cultural differences including language, food, customs, and social patterns when studying in a country other than their native country. International student-athletes must make many of the same adjustments as their international student counterparts, although some key differences do exist. For example, international student-athletes are often placed immediately within a team structure, aiding with socialization. Lack of such a socialization unit is often cited as a problem for many international students. In addition, many international students must pay for their own expenses while studying abroad, often without the ability to work in the United States because of a lack of the proper visa. International student-athletes, particularly at the NCAA Division I level, however, often have a full athletic scholarship, allowing them more financial freedom. Still, international student-athletes encounter many of the same barriers as the international non-athlete students, many of which are outlined by the following researchers.

Westwood and Barker (1990) examined the relationship of academic and social adjustment for international college students who participated in a peer-partnering program with host nationals compared to those who did not. The researchers in this study sought to find if international students would do better academically, be less likely to drop out, and made more successful social adjustments if they were coupled with a host national student who would act as a peer partner.

The researchers surveyed four cohorts of international students, three at the University of British Columbia and one at an Australian university. Each cohort began 
their college career in a different academic year between 1984 and 1987. In the three Canadian groups, international students volunteered for a peer-pairing program in which a host national student was assigned to meet or be in contact with the international student on a regular basis. For every international student participating, the researchers selected another international non-participant with similar demographic variables such as age, sex, year, and program of study. At the end of the year, participants' grade point averages (GPAs) and drop-out rates were compared, via independent samples t-tests, to the non-participants.

The first cohort contained $25(N=50)$ participants, the second, $41(N=82)$, and the third, $31(N=62)$. The researchers changed the design to a completely experimental format in the fourth Australian group, randomly assigning 24 international students to a host national peer partner, and asking 23 international students to form an un-partnered control group. Results of the t-tests in all four cohorts were statistically significant at the .01 level for both GPA and drop-out rate. In the control groups, 19 of 120 international students dropped out, while just 2 of 121 students in the experimental groups left school. All participants in the Australian cohort also completed the Companion Check List (Furman \& Bochner, 1982), which is a measure of social adjustment. The results of the check list from both the control and experimental groups were analyzed through frequency comparisons which revealed no significant findings.

The results of the study further illustrated higher adjustment levels for international students who are given assistance and social companionship during their transition period in a new country. The results, however, must be accepted with caution because many participants in the experimental groups chose to have a host national 
partner. By making this choice, participants might have possessed other personality traits assisting with ability to adjust which were not accounted for in this study. An example might be high motivation. The researchers also pointed out possible benefits for the host nationals when peer partnering, which may be an area for further study.

Parr, Bradley, and Revathi (1992) investigated major concerns of international students at United States universities and how those concerns related to the students' feelings about their experiences. Previous research listed variables troublesome to international students and traits or background information affecting international students' acculturation or ability to adjust to a new environment. The investigators stated that none of the few studies conducted were large enough to procure generalizable results. In their study, Parr et al. randomly selected 100 United States universities and mailed five questionnaires to the schools' student personal office. The mailing included a request of school officials to distribute the surveys to the first five international students who entered their office. The researchers received 163 completed surveys, a return rate of $34 \%$.

The survey instrument listed 11 demographic variables, 40 items related to concerns, and 15 items related to feelings. Participants measured concern items on a 6point Likert-type scale anchored by great concern (1) and no concern (6). Those same subjects measured feeling items on a 6-point Likert-type scale anchored by very frequently (1) to very infrequently (6). The researchers ran a factor analysis on the concern items, reducing them to: (a) cultural differences, (b) schools, (c) practical necessities, (d) extended family, (e) living essentials, (f) contact with homeland, (g) finances, (h) time, (i) socialization, and (j) discrimination. The overall Chronbach's alpha 
score for concerns was .94. The researchers ran the same procedure on the feelings items, dividing those factors into three items: (a) positive feelings, (b) negative feelings, and (c) social feelings, with an overall Chronbach’s alpha rating of .81. Using descriptive statistics, the researchers stated extended family (3.12), cultural differences (3.35), finances (3.35), and school (3.42) most concerned international students, while practical necessities (4.25), socialization (4.16), and time (4.03) were of least concern. A Pearson $r$ correlation revealed significant relationships between concern factors and feelings.

The researchers also utilized a MANOVA, with two dependent variables of concerns and feelings, and independent variables comprised of the demographic information collected. The test revealed significant findings only concerning the independent variable length of stay in the United States. Students spending less than a year in the United States identified significantly more positive feelings than students who had spent one to two years in the country. Results did demonstrate, however, no significant difference between positive feelings in the first year and positive feelings for more than two years, indicating a boomerang or U-shape curve. Previous research by Lysgaard (1955) found a similar U-curve result.

Kaczmarek, Matlock, Merta, Ames, and Ross, (1994) compared college adjustment between international students and domestic students. The researchers wanted to determine if a difference existed between expected adjustment and actual adjustment of international students and if this difference was significantly different than that experienced by domestic students. While several researchers identified differences in adjustment for international college students compared to domestic students, Kaczmarek et al. were the first to use the Student Adaptation to College Questionnaire (SACQ; Baker 
\& Siryk, 1989), which is widely regarded as the most reliable and valid measure of student adjustment to college, with a population of international students.

The researchers in this study administered the SACQ to 76 international students at a single university at the beginning of the fall semester. Students were requested to complete the instrument again nine weeks later after they had time to adjust to college life in the United States. Of the 76 participants, 29 completed usable surveys a second time. The researchers also administered the survey to 57 United States-born students at the end of the nine week period, giving the researchers comparable post-test data of a comparison group.

One of the researchers' goals was to test the "matriculant myth", namely the tendency for students to have greater expectations than what they can realize when experiencing a particular phenomenon. No significant differences were found, however, in the international students' pre- and post-experience questionnaire scores. The researchers then utilized t-tests to examine group differences between the international student post-experience results compared to domestic students. Statistically significant results $(p<.02)$ were found on two of the SACQ's four subscales: social adjustment and institutional attachment, with international students scoring lower on both scales. Kaczmarek et al. (1994) also ran t-tests between the pre- and post-experience scores of international students and found a statistically significant result between the demographic question "Is there a faculty member who has been a significant help to you?" and one subscale score; personal-emotional adjustment. International students who answered "yes” to the demographic question scored lower on the adjustment subscale. 
The results of this study revealed no significant difference between what international students expect of their experience and what they actually go through in terms of adjustment, rejecting the "matriculant myth." The researchers suggested the two subscale (social and institutional attachment) differences between international and domestic students came as a result of cultural differences and the difficulty international students have in becoming involved in campus activities or school leadership roles. They also mentioned prior research suggesting international students tend to socialize with other international students, thus limiting their social adjustment ability at a United States university (Surdam \& Collins, 1984). The researchers explained the relationship between having a faculty member who helped and low personal-emotional subscale scores by stating international students who sought help were already experiencing significant personal stress. The researchers felt the SACQ is appropriate for gauging international student adjustment and future research should include larger samples.

Luzzo, Henao, and Wilson (1996) examined the academic and social needs of international students. Using qualitative methods, the researchers had international students complete an open-ended questionnaire consisting of 12 questions, then gathered all participants for a focus group interview one week later to discuss their answers to the survey. The sample consisted of eight participants who all attended a single United States university but were from six geographically diverse nations. Questions on the survey dealt with academic, social, and cultural adjustment issues, such as "How has your social life changed since starting college?” and "How has the university addressed your social/cultural needs?” One of the researchers moderated the focus group interview, which was also videotaped. 
The researchers separately analyzed all the written responses from the survey and the oral responses from the videotaped session, identifying themes emerging from the data. After this individual analysis, themes were compared and discussed among the researchers. Six themes developed out of the analysis: (a) overall satisfaction with the university meeting international student academic needs, (b) active involvement with campus activities which were viewed as an important part of campus life, (c) many friendships formed with people of diverse ethnic backgrounds, which was also positively viewed by the international students, (d) good experiences with dorm life, but dissatisfaction with food selection in dining hall, (e) dissatisfaction with intramural sport offerings, and (f) desire for the university to provide more transportation options.

The researchers suggested their study could result in actionable changes in student service options for international students, such as more global variety in food and sport options on campus. They also called for further qualitative research regarding academic and social needs of international students.

Ladd and Ruby (1999) examined learning styles of international students. Differences in learning styles affect how quickly and effectively students learn. The teaching methods used by many United States university professors vary from those employed by teachers in many other countries. The academic adjustment process of international students in United States schools can be improved through a better understanding of United States teaching methods by the students and of learning styles of international students by professors.

In their study, Ladd and Ruby (1999) administered the Canfield Learning Styles Inventory to $35(N=35)$ international students enrolled in an MBA program at an 
accredited American Assembly of Collegiate Schools of Business state university. The assessment measured four categories: (a) conditions for learning, (b) area of interest, (c) mode of learning, and (d) expectation for course load. Descriptive statistics showed 80\% of respondents learned through lecture in their home country, while another 14\% learned through reading. Results from the Canfield Learning Styles assessment, however, demonstrated students preferred learning through direct experience. Other critical results from the Learning Styles evaluation included: (a) international students desired to identify and pursue goals related to their interest, (b) international students sought warm, personal relationships with teachers, (c) international students wanted to learn about human behavior and jobs like personal interaction occupations such as sales and teaching, (d) international students desired direct contact with course materials and topics studied in class, (e) international students wanted to imitate the behaviors of their instructors, and (f) international students predicted they would achieve high grades in their course work.

The investigators also offered several suggestions for helping international students succeed academically at their United States institution. They cited CastroAbad's (1995) definition of the difference between assimilation, a process where new group members lose their distinguishing characteristics to blend in with the dominant group, and acculturation, which is the process of adapting to a new culture without losing an original cultural identity. Ladd and Ruby (1999) suggested instructors encourage international students to acculturate into their new environment through means such as joining clubs or participating in social organization, while discouraging assimilation. They also recommended professors slowly develop the concept of class participation to international students, who are likely used to only lecture methods of instruction. 
International students might also require additional help with American idioms and explanation of non-lecture teaching methods used in United States college classrooms. Rajapaksa and Dundes (2002) examined the adjustment process of international students compared to those of United States-born students when attending United States universities, with an emphasis on the ability of satisfactory social networks to predict high adjustment levels. The study focused specifically on two aspects of acculturation: (a) psychological adjustment and (b) sociocultural adjustment. The researchers used a two-page survey instrument to assess adjustment levels of international students $(N=$ 182) attending 12 different schools. The instrument was based on scales developed by Archer, Ireland, Amos, Broad, and Currid (1998) to evaluate homesickness and by a scale developed by Shin and Abell (1999) to measure homesickness and contentment of Asians. The investigators also administered a one-page version of the instrument, which eliminated questions about travel abroad for school, to 100 domestic students attending a small liberal arts school. The researchers received a nearly 100\% response rate from all participants.

International students in the study represented six continents, with the highest percentages coming from Europe (23\%), India and Sri Lanka (17\%), and Asia (14\%). Other important demographic variables of the international students included: (a) $45 \%$ were male, (b) $74 \%$ were 22 years old or younger, (c) $66 \%$ had spent two years or fewer in the United States. International students pursued a wide range of majors, although a large percentage (54\%) were in business or economics fields. In addition, $60 \%$ of international students lived in dorms or in college-owned housing, while only $5 \%$ funded their education through scholarships. Key results to questions regarding the main 
adjustment variables included: (a) 76\% felt they were not discriminated against by Americans, (b) 68\% agreed Americans were open to their native culture, (c) 76\% felt accepted by Americans, (d) 62\% found the American culture and its freedoms refreshing, (e) $79 \%$ were content with their adjustment, (f) $70 \%$ did not feel lonely or homesick, and (g) $46 \%$ did feel they left a part of themselves at home.

Employing a two-tailed difference of proportions test, the researchers found significant differences between international students and United States-born students in terms of contentment, loneliness, homesickness, and leaving a part of themselves at home. The more relevant findings in this study, though, revolved around the idea that the number of friends and type of friendships both international and domestic students had did not correlate with their ability to adjust. The findings showed $42 \%$ of international students had no close friends, while another $42 \%$ reported having only one to three close friends in the United States, which did not correlate with the previous statistics depicting international students' comfort and acceptance levels. Past research has shown a close relationship with social network satisfaction and ability to adjust, but Rajapaksa and Dundes' (2002) work demonstrated close friendships were not strong predictors of social networking. The researchers suggested administrators wishing to assist international students on their adjustment should focus less on creating strong friendships, but rather on other aspects of social networks.

Chapdelaine and Alextich (2004) explored and tested a model of culture shock first introduced by Furnham and Bochner (1982). The researchers defined culture shock as "the multiple demands for adjustment that individuals experience at the cognitive, behavioral, emotional, social, and physiological levels, when they relocate to another 
culture” (p. 168). The original Furnham and Bochner model based international students' struggles with cross-cultural interactions on not understanding the social skills or expectations of their new environment and not receiving adequate socialization input from members of the host country. Chapdelaine and Alextich referred to this concept as social difficulty. The investigators, however, pointed out two major limitations of the Furnham and Bochner model: (a) culture-specific social skills were difficult to identify with any degree of specificity, and (b) cross-cultural social difficulty was compared between the international students and native students. They suggested a more relevant comparison would be between the international students' perceived level of social difficulty in their home country and in their new country.

In their study, the researchers presented several hypotheses. Based on their review of the literature, Chapdelaine and Alextich (2004) hypothesized international students would report higher levels of social difficulty in their new country, Canada in this study, than in their native country. Secondly, they suggested a series of concepts which would expand the Furnham and Bochner (1982) model. They presented these suggestions through a series of hypotheses: (a) higher levels of social interaction with hosts would result in lower culture shock, (b) higher levels of cross-cultural differences in social interaction would link to high levels of culture shock, (c) high levels of cross-cultural difference in social interaction would link to lower levels of social interaction with hosts, (d) size of co-national group would correlate positively to social interactions with hosts, (e) family status in host country would be negatively correlated with social interaction with hosts, and (f) previous cross-cultural experience would be associated with lower levels of culture shock. 
The researchers used a population sample of 195 international graduate students from a single university in Western Canada. Of that population, 156 agreed to participate, a response rate of $80 \%$. Participants came to a testing center in groups of four or five to take a series of written surveys, namely the Cultural Distance Index (CDI) developed by Babiker, Cox, and Miller (1980), the Social Interaction Scale (SIS), developed and pilot tested by the researchers, and the Revised Social Situations Questionnaire, original constructed by Furnham and Bochner (1982), but with modifications developed by the researchers. Participants also shared demographic information, which revealed a population of all male students (international female students were not selected because of their underrepresentation at the school) comprised of 31 students from China, 29 from India, 22 from Iran, and 74 from other countries. Most of the participants were between 25 and 34 years old (69\%), while they were split rather evenly between married (55\%) and single (44\%). Over $70 \%$ of participants stated they had no other previous crosscultural experiences before their stay in Canada.

The CDI test compared different aspects of the students' social lives in Canada versus their social lives in their home nation. Participants answered a series of Likert-type questions regarding social life differences anchored by 1 (no differences at all) to 5 (very different). The CDI was tested for reliability and produced a Cronbach's alpha score of .85. The 18-item SIS measured social interaction between the international students and Canadians, co-nationals, and other international students. The 35-item RSSQ measured social difficulty in Canada and in the home country. Researchers produced a score by subtracting native country score from the Canadian score. The two subscales were tested for reliability resulting in Cronbach’s alpha scores of .89 and .84 . 
Chapdelaine and Alextich (2004) ran a paired t-test, $t(139)=11.99, \mathrm{p}<.01$, on the scores of social difficulty in Canada and in their home nation, and were able to confirm their hypothesis of more difficulty in the foreign country. The investigators did note participants had low mean social difficulty scores in both their home country and Canada, so despite the significant difference, students did not seem to struggle much with social difference. To test their remaining hypotheses, the researchers used path analysis. Results demonstrated problems with the Furnham and Bochner (1982) model. Post hoc analysis revealed the specific errors were located in the hypotheses of higher levels of cross-cultural differences linked to higher to levels of culture shock and higher levels of previous cross-cultural experience would link to lower levels of culture shock.

Constantine, Anderson, Caldwell, Berkel, and Utsey (2005) examined the cultural adjustments and issues faced by international students from Kenya, Nigeria, and Ghana while attending United States universities. International students submerged in a new culture confront several obstacles, often referred to as culture shock, and must find ways to cope with their new environment and expectations. This experience is called acculturative stress, and if not understood and dealt with properly, can cause social, psychological, and even physical problems. African international students in particular face unique adjustments for several reasons: (a) their race or skin color, (b) the Africancentered concepts of communalism and group survival versus the individualism often expressed in United States university students, and (c) the long distance from their homeland and their sense of isolation. Previous research indicated African students incorporate several different means to cope with these stressors, including intense work habits and seeking help from other African students, campus staff, and professors. 
Constantine et al.(2005) sought to uncover the adjustment experience of these students through qualitative study. The researchers used Hill, Thompson, and Williams' (1997) recommended technique of Consensual Qualitative Research (CQR) for this study. They selected 12 participants for their research, four each from Kenya, Nigeria, and Ghana. All investigators collaborated to develop semi-structured interview questions, based on the literature review, and pilot tested the questions with Ghanaian undergraduates. Researchers took turns interviewing the participants individually and recording responses, which were then coded and compared. After analysis and discussion, the research team settled on seven broad categories of responses: (a) presojourn perceptions of the United States, (b) postsojourn perceptions of the United States, (c) cultural adjustment problems in the United States, (d) responses to prejudicial or discriminatory treatment, (e) family and friendship networks, (f) strategies for coping with cultural adjustment problems, and (g) openness to seeking counseling to address cultural adjustment problems. All responses from participants were placed into subcategories under these seven concepts and labeled by frequency. If all 12 participants made a comment regarding a subcategory, the researchers labeled it "general", while if 6 to 11 participants mentioned the statement, it was marked “typical”. Statements appearing three to five times were called "variant”, while responses which could only by linked with one or no other responses were disregarded.

Participants revealed their pre-sojourn thoughts of an education in the United States being superior to those on offer in their native land. After arriving in the United States, they felt White Americans controlled most of the power in the country and native university students, and even faculty, did not possess a strong awareness of global issues. 
Cultural adjustment issues for the participants included discriminatory and prejudiced treatment through racial slurs and a demonstrated fear of Africans. Participant responses to such reactions varied from becoming angry to efforts of enlightenment. Participants did report strong relationships with family and support from friendships in the United States. The most common support mechanisms for the participants when faced with acculturative stress were relying on support from family and friends, internalizing the problems, exercise, and sleep. The researchers found participants were not very open to the concept of counseling. In conclusion, the investigators felt school counselors needed to become more aware of the particular issues international African students face and to find alternative ways to introduce them to coping mechanisms besides traditional counseling sessions.

As several studies outlined above, international students must make many adjustments when studying in another country. Among the most important factors in successful adjustment is an ability to adapt socially in their new environment. While this fact is no different for international student-athletes, international student-athletes do possess a major advantage compared to their international student counterparts. Studentathletes join a team upon their arrival to the United States which provides an immediate socialization network. This is a key difference between international students and international student-athletes and provides a good basis for the theory international student-athletes adjust socially better to college.

\section{Student Adjustment to College}

Whether growing up in the United States or in another country, all students make some social, cultural, and academic adjustments as they transition from high school to 
college. This transformation is often a critical time for the maturation of adolescents into adults. Several researchers have investigated this transitional period and attempted to develop empirical measures to detail the adjustment process. One team of researchers stands out in particular for their work in developing student adjustment instruments. Baker and Siryk $(1984 ; 1986 ; 1989)$ have looked at college student adjustment for over 20 years, and have developed the Student Adaptation to College Questionnaire (SACQ) scale used by, among others, Ridinger and Pastore (2000a; 2000b) to measure international student-athlete adjustment.

The following few studies examine work done in the area of student adjustment assessment. Baker and Siryk (1984) sought to develop an instrument to measure student adjustment to college. A review of literature showed past researchers employed a variety of instruments, measuring a range of adjustment dimensions. Most previous assessments were not held to high levels of reliability or validity. Baker and Siryk stated the most comprehensive student adjustment instrument prior to their work was the College Inventory of Academic Adjustment (CIAA) which was developed by Borrow in 1949. Through the researchers' examination of the literature, four themes of student adjustment emerged: (a) academic, (b) social, (c) personal/emotional, and (d) institutional or goal commitment.

In their development of a comprehensive measurement scale, Baker and Siryk (1984) constructed a 52-item instrument, comprised of four subscales measuring their four dimensions of student adjustment. They administered their survey to freshman at Clark University during the first and second semesters over the course of three successive years. In the first year, 233 students returned the instrument in the first semester and 185 
in the second. In the second year of the test, 217 and 172 students completed the survey during the first and second semester, respectively. The third year resulted in 171 and 168 returned surveys over first and second semester, respectively. The first semester return rates ranged from $64.2 \%$ to $72.7 \%$ while the second semester return rates ranged from $54.8 \%$ to $63.1 \%$. All six times the survey was administered, overall reliability produced a Cronbach’s alpha score of .92 or better. On individual subscales, academic scores ranged from .82 to .87 , social scores from .83 to .89 , personal/emotional scores from .73 to .79 , and general subscale scores (which measured institution or goal commitment) from .84 to .88 .

To determine validity of the instrument and sub-scales, the researchers compared survey results to participant statistics of other data available such as (a) attrition rates, (b) appeals for psychological services, (c) freshman grade point average, (d) election to academic honor societies, (e) participation in social activities, and (f) applications for dormitory supervisor positions. Correlations were run between survey scores and these six measurements resulting in several significant results. Attrition produced significant ( $p$ $<.01)$ negative correlations with the social subscale and the use of psychological services produced significant negative correlations with the personal/emotional subscale. Grade point average and election to the honor society produced significant $(p<.01)$ negative correlations with the academic subscale in five and four of the tested cohorts, respectively. Results of ANOVA tests showed a significant $(p<.05)$ positive relationship between high social subscale scores and selection as dormitory assistants two years later.

Study findings led the researchers to believe their adjustment scales were measures in the right direction, as high levels of validity and reliability were established. 
The researchers did caution the alpha coefficients for the personal/emotional subscale were a little low and the sample represented just one college.

Baker and Siryk (1986) re-examined their student adjustment to college scale (1984) to test how intervention with students who had indicated extreme scores on the original scale, affected attrition and adjustment levels of those students. The researchers conducted several studies to develop an instrument to measure the multi-faceted dimensions of student adjustment to college. Their survey assessed four such dimensions: (a) academic adjustment, (b) social adjustment, (c) personal-emotional adjustment, and (d) institutional attachment. In this particular study, Baker and Siryk administered the survey to a cohort of freshmen in their first semester of school, then personally interviewed many of the participants whose scores were at the extremes of adjustment to college. The survey was administered a second time to the same students late in the spring semester to gauge the affect of the intervention.

All participants were students at Clark University. Eight weeks into the first semester, the researchers sent instruments to all 549 members of the 1981 freshman class, and had 216 surveys returned, a rate of 39.3\%. Past studies documented the reliability of the instrument, as Baker and Siryk (1986) reported Cronbach alpha reliability scores of .81 or higher for all scales in their previous work. Students reporting at least one high-end or low-end adjustment score on any of the scales in this study were put in two groups, with 86 students in the high-end, and 90 in the low-end. Both groups were then split into equally distributed sub-sections, based on gender, producing four groups. The researchers extended invitations to all subjects in one high-end group and one low-end group to participate in an individual interview to discuss the instrument and their feelings about 
their adjustment to college. Of the 39 low-end students invited for an interview, 36 showed up, while of 37 high-end students invited, 31 showed up. The researchers explained to the interviewees how the scales were constructed, why the participants were selected, and if the participants remembered what scales they had marked high or low. Students were also questioned whether anything had changed since the survey, which might affect their adjustment, and how they felt about the items on the instrument. After Baker and Siryk completed their interviews, they sent out their adjustment survey a second time to all students who filled out the first survey.

All scales in both administrations of the survey produced coefficient alpha levels of .79 or higher. The researchers found several interesting results. One major finding was the high turnout of participants who volunteered for individual interviews. These interventions were not only helpful to receive feedback on the survey instrument, but also offered an important insight regarding satisfaction with the college experience. Tests of ANOVA revealed significant differences in several subscale scores between the first test and the second test for those participants who were interviewed compared to those who were not. Thus, the researchers suggested intervention may improve college student adjustment levels, particularly for students who indicate low levels of adjustment early in their college careers. Statistics also showed 8 of 36 low-adjustment students who were interviewed left the university by the end of the eighth semester, an attrition rate of $22.2 \%$. Low adjustment students who were not interviewed left school at a $44.7 \%$ rate (17 out of 38), a significant difference with a $p$-value of less than .05. This may be an indication that college student adjustment and retention can be improved simply by 
giving students a chance to discuss their adjustment situation with a counselor or other college personnel.

Leong and Bonz (1997) examined the impact different coping styles had on student adjustment to college. The researchers employed Tyler’s (Tyler, Brome, \& Williams, 1991) model of psychosocial competence, testing how different coping strategies affect four different levels of adjustment: (a) academic, (b) social, (c) personal/emotional, and (d) attachment/goal commitment. These four dimensions emerged from the work of Baker \& Siryk (1984).

To test their hypotheses, the authors surveyed $161(N=161)$ college freshmen at an Ivy League school. Their sample consisted of 56\% males and 44\% females, while 60\% of respondents were white, 27\% Asian, 4\% African-American, 3\% Hispanic, 1\% Native American, and 2\% did not respond to the race question. Leong and Bonz (1997) did not report a response rate.

Participants completed Baker and Siryk’s (1989) Student Adaptation to College Questionnaire (SACQ), which assessed levels of college adjustment in the four dimensions as listed above and provided the dependant variable scores in the current study. The SACQ results had alpha reliabilities of .80 for academic adjustment, .80 for personal/emotional adjustment, .79 for social adjustment, and .52 for attachment/goal adjustment. To assess coping style, which was the independent, or predictor, variable in the study, the researchers utilized Weintraub, Carver, and Scheier’s (1986) COPE survey, which measures various ways of responding to stress. The COPE instrument reveals 12 coping strategies: (a) positive reinterpretation and growth $-\alpha=.75$, (b) mental disengagement $-\alpha=.43$, (c) focus on and venting of emotions $-\alpha=.78$, (d) seeking 
social support for instrumental reasons $-\alpha=.74$, (e) active coping $-\alpha=.74$, (f) coping through denial $-\alpha=.62$, (g) turning to religion $-\alpha=.94$, (h) behavioral disengagement $-\alpha=.52$, (i) seeking social support for emotional reasons $-\alpha=.86$, $(\mathrm{j})$ acceptance $-\alpha=$ .68 , $(\mathrm{k})$ suppression of competing activities $-\alpha=.63$, and (l) planning $-\alpha=.82$. The researchers then regressed adjustment scores on coping scores through four (one for each adjustment dimension) stepwise multiple-regression analyses. T-tests were also conducted to determine differences in adjustments and coping styles based on gender. No significant differences were found in adjustments, but females did report statistically significant higher scores for the coping strategies of focus on venting of emotions, seeking social support for instrumental reasons, and seeking social support for emotional reasons.

Analyses revealed active coping was a positive predictor of academic adjustment ( $\beta=.27)$ and personal/emotional adjustment $(\beta=.15)$. Suppression of competing activities negatively predicted academic adjustment $(\beta=-0.19)$ and focus on and venting of emotions negatively predicted personal/emotional adjustment ( $\beta=-0.23)$. No other coping strategies were statistically significant predictors of any dimension of college adjustment.

Implications for this study included the rejection of the notion, at least for highfunctioning freshmen at this Ivy League university, that males employ more active coping strategies than females. Students using active coping strategies did have higher levels of adjustment and the strategy of venting emotions had a negative impact on adjustment. A somewhat surprising finding was the suppression of competing activities, in other words, neglecting other activities to focus specifically on one thing, had a 
negative impact on adjustment. For practitioners, this study implies an emphasis on encouraging students to use active coping strategies.

The previous studies outline some of the research which has been done examining the ability of United States students to adjust to college. Baker and Siryk have been at the forefront of this research and have formulated four measurements of student adjustment to college: (a) academic, (b) personal-emotional, (c) social, and (d) institutional attachment. How students adjust to college in these four factors gives an excellent indication of how student adjust to college overall. Most studies on college adjustment utilize Baker and Siryk (1989) measurement tool, the Student Adaptation to College Questionnaire (SACQ), which has proven through numerous studies to be very reliable and post high validity scores. The SACQ has been used to measure both international students' and international student-athletes’ adjustment to college and will be one of two instruments used in this particular study.

\section{International Sport Policy Analysis}

Before examining international student-athlete adjustment, it is important to understand the context in which international student-athletes develop. This review of international sport policy literature is divided into four components: (a) national sport policy framework and analysis, (b) specific national sport policies, (c) the club-based sport system, and (d) youth participation in club-based sport. Different nations emphasize different aspects of sport policy, some with heavy governmental regulation and others with very little.

These sport policies are important to society in general, and sport managers specifically, for many reasons. Sport policy legislation and documentation dictates how 
participants access sport opportunities, which sport organizations will have access to limited resources, and how citizens' health and feelings of national pride will be affected (Chalip et al., 1996). In some countries, the opportunity to play sport in school, in sport clubs, or recreationally, is available to all. In others, such opportunities are rare or nonexistent. For many countries, success of elite sport in international competition is of utmost importance, with large sums of money funneled through national sports bodies in an effort to develop the world's top athletes. Other countries use more of a "Sport for All” model providing sporting opportunities for all citizens, regardless of age, gender, ability, religion, refugee status, social economic class, or sexual orientation have become the prime concern. Where a nation places its collective emphasis in sport policy can dramatically affect the health of its children, its image of strength and power on a global scale, and its economy, ranging from sport tourism income to the cost of education and health care.

Beyond the decisions made by policy makers are the methods in which sport managers direct sport opportunities. For many youth throughout the world, sport opportunities begin through experiences in schools and clubs. The standard of coaching, the condition of the sports venues and equipment, the financial and time costs of involvement, the opportunity for participation and advancement, and the creation of an enjoyable experience all play important roles in determining whether youth will continue to play sport and remain physically active into adulthood and throughout their lifetime (Palm, 1991).

The major issues included in the following literature review extend from comparative sport policy analysis, which looks at issues more theoretically, to the 
structure of youth sport in clubs and schools. What events and paradigms historically, economically, and theoretically led to the formation of particular policy and what have been the results of those events and frameworks? This study examined those issues in several countries, in an effort to explain how some sport policies have developed. This investigation also examined different tracks available for youth sport participation, how youth become involved and stay involved in sport, how long they remain involved in sport, their options as they become older and more skilled, and how participants feel about their opportunities.

The studies addressed in this section examine the theoretical and practical concepts behind current national sports policy. The historical and political context of every nation frames its sport policy. This study addressed such frameworks first with conceptual ideas, followed by specific national policies. National sport policies examined, at least in part, in this study include current and former countries such as New Zealand, Canada, England, East Germany, West Germany, the Soviet Union, France, Finland, the Netherlands, Greece, and the United States.

\section{Conceptual Frameworks}

This line of research began by examining theoretical frameworks developed to analyze comparative sport policies. These frameworks often cite examples from specific countries, but address the broad issues which are necessary to understand before critiquing specific national sport policy. These articles discuss the importance of historical and political context of sport policy development and look at the major building blocks for sport policy development, such as economics, opportunity for all, and elite sport construction. 
Harvey, Beamish, and Defrance (1993) developed a framework for the comparative analysis of exercise policies in "welfare" states, or countries where the "state accepts responsibility for the provision of comprehensive and universal welfare for its citizens” (Spicker, 2006, Introduction section, para 1), setting up future multi-nation studies. Exercise policies refer to specific national policies pertaining to the body and initiated to develop physical fitness and sport practices among a nation’s citizens. Harvey et al. began by listing the considerations necessary for a comparative analysis, then delved into a "Society-State" framework. The researchers used several historical examples from exercise policy of Canada, England, France, and Germany, to illustrate their framework and concluded with directions for further studies.

To construct a comparative analysis, the researchers stated several premises which needed to be accepted: (a) "welfare states are first and foremost historical institutional formations that evolve under circumstances specific to each state, (b) physical exercise policy is to be understood in the context of the state's formation and the welfare state's development, (c) physical exercise is a phenomenon which also evolves under specific historical perspectives, and (d) study of the transformation of the meaning of physical exercise, as well as the evolution of the actual initiatives of the state with regard to physical exercise, in the context of state formation” (Harvey et al., 1993, p. 55). Harvey et al. suggested a framework incorporating both society-related variables and state-related variables.

The society-related variables included social classes, the women's movement, and ecology concerns. The researchers used examples such as the passing of the English Physical Training and Recreation Act of 1937 and Canada's Dominion-Provincial Youth 
Training program (1937). In the post-depression era, both England and Canada had severe unemployment. The English act was meant to control the behavior of unemployed youth, but also to increase accessibility to leisure activities, while the Canadian measure was simply an effort to produce more physically fit and capable workers. The antiapartheid movement was a society-related variable affecting a country's (South Africa's) exercise policy. Another society-related variable was a nation's political economy. The authors used Canada as an example. There, policy-makers emphasized elite sport and saw sport as an industry, which in turn has meant Canada's exercise policy lags behind places like Scandinavian countries, where exercise and "Sport for All" is viewed as a public right.

An additional society-related variable is the collective, mental image of the "state". The example used here again goes back to post-depression England, where exercise policy was rooted in ability to do work, employability, and laborer health. By comparison, France, which was not hit as significantly by the depression, developed exercise policy as a reaction to educational reform, with more emphasis on mind-body wellness. The final variable under the society-related heading is "a given social formation within the international economic order" (Harvey et al., p. 58). In other words, exercise policy is affected by a nation's status in the world economy. For example, the economies of the United States, England, and Canada, are closely linked to each other and are more conservative, leading to more privatization of physical exercise services.

In examining state-related variables, the investigators looked at the history of a state and its political structure. They cited the most important state-related variable as state actors. Different states have different means of electing or assigning state actors, 
which ultimately affects exercise policy. One such example is in Canada, where physical educators and sport managers worked their way into key political positions, re-shaping the country's exercise policy.

In summary, the researchers concluded it was important to understand a nation's political history before performing a cross-nation comparative analysis. They suggested future studies will have difficulty truly capturing the complexity of the issue through quantitative studies, particularly if an historic perspective is not addressed. They believe their framework set the stage for further studies designed to truly "understand the social dynamic of policy formation in the area of physical exercise.” (Harvey et al., 1993, p. 61)

Chalip (1995) examined the recent history of policy analysis and linked it specifically with sport policy analysis. He illustrated formal methods for interpretive and critical policy analysis by using a case study of the United States Amateur Sports Act. During his description of policy analysis, Chalip discussed two different policy intervention techniques used by sport managers: (a) lobbying, which consists of direct efforts to influence government actions, and (b) issues management, which involves monitoring content of legislation and ensuring measures fulfill the goals with which they were intended instead of becoming political tools.

Chalip (1995) outlined five elements necessary to execute and interpret a policy analysis: (a) focusing events, (b) operative legitimations, (c) problem definitions, (d) problem attributions, and (e) decision frames. Chalip used the development of the United States Amateur Sports Act to help define and explain this analytic framework. The focusing event, or the issue attracting national attention, was the 1972 Olympics. At those Games, the United States men's basketball team lost the gold medal in a controversial 
last second decision, two United States sprinters were disqualified after following an incorrect schedule, a United States pole vaulter was barred from using his fiberglass pole, and a United States swimmer was stripped of his gold medal for taking a banned substance contained in asthma medication recommended by his trainer.

The operative legitimation, or issue requiring legitimate government intervention, was national prestige, while the problem definition was to beat Soviet athletes without using their means. The problem attribution was administrative incompetence, and the final decision frame was administrative rationalization, meaning government intervention without taking over the United States Olympic Committee. Using his framework, Chalip (1995) concluded the United States government did make efforts to fix the problem, first with the appointment of the Commission on Olympic Sports in 1975, followed by that Commission's report in 1977, which ultimately led to the creation of the Amateur Sports Act (ASA). Chalip's conclusion was not to critique the ASA policy, but to demonstrate the necessity for sport managers and organizations to analyze policy that can affect them by utilizing a useful framework.

Green (2005) investigated a pyramid model of sport development, critiquing its three levels: (a) mass participation sport, (b) competitive sport, and (c) high performance sport, and demonstrated the United States sport development system through a case study of USA Volleyball (USAV). Sport systems can be framed by a pyramid model which starts with recruiting athletes from a large population, then moves to retaining those recruited athletes (which is a significantly smaller population), and is completed at the top by developing a system of transition where athletes have the opportunity to advance to an elite level. When properly developed, the sport system can achieve two primary 
objectives: (a) increasing the number of participants and (b) enhancing the quality of the sport performances.

Green (2005) cited two reasons people become involved in sport, recruitment and retention, which she sub-divided into three categories: (a) motivation, (b) socialization, and (c) commitment. The recruitment component is important to sport system development because it develops initial interest in sport for young athletes. Using the USAV case study, Green illustrated that while pre-adolescent athletes typically feel a commitment to their sport, volleyball development in the United States does little to encourage playing opportunities before middle school.

Green (2005) elaborated on the three dimensions of retention, starting with motivation. Citing several studies, she illustrated many motivations for a young athlete to remain involved in a sport, items such as (a) social interaction, (b) fitness, and (c) skill development. Green noted, however, little has been researched regarding the nature of benefits people perceive in sport, how those benefits come to be valued, or how people come to perceive personal control over those benefits. She also stressed the importance of developing programs catering to multiple motivations, appealing to both competitive and participatory levels, as well as keeping young athletes involved.

Next, Green (2005) examined the importance of socialization, which often links recruitment to commitment. Socialization includes the role an athlete fills on a team, and a sense of belonging or membership in the sport subculture. The term undermanning (Wicker, 1979) is defined at this stage. Undermanning refers to the concept of a sport organization offering enough opportunities for all participants to have a role, instead of a selective procedure where some athletes are turned away. 
In a third dimension of retention was commitment. Green (2005) noted when athletes committed to a sport, their participation in other activities declined. Stevenson (1990) looked at specialization in sport and found commitment to a sport involves both the potential for success in the sport and the other people involved in the sport. Program design should incorporate not only skill building and avenues to improve, but also social and support activities. Green also stated "goal planning (as opposed to mere goal setting) is something that needs to be trained from the outset.” (2005, p. 243)

The final stage Green (2005) examined was athlete transition, an area rarely researched. She listed three common problems for advancement within pyramid-based systems: (a) the existence of sequential levels in a sport system does not always mirror athlete progression, (b) communication between levels breaks down, and (c) an absence of social and material assistance for advancing athletes. In the United States, sport policy is not relegated to government organizations. Therefore, various and competing developmental sport organizations do not neatly link together. Green also outlined policy to ease athlete transition and looked at six symptoms of culture shock which can occur during poor athlete transitions: (a) a sense of stress, (b) feelings of loss and deprivation, (c) fears of rejection, (d) confusion about role identity and expectations, (e) disorientation engendered by unanticipated expectations in the new cultures, and (f) feelings of inadequacy (Furnham \& Bochner, 1986). The researcher concluded by remarking conceptual frameworks for United States’ sport systems exist, but are rarely implemented in program planning, often to the detriment of the United States athlete. 


\section{National Sport Policy Analysis}

Several researchers in various countries have studied specific national sport policies. A handful of themes emerged from these various studies, including national interest in elite sport performance (winning medals in the Olympic Games and international competitions), promotion of healthy lifestyle and active youth participation, and shifting bases for growth in sport popularity and economy. The issue of political power, which shapes nearly all national sports policy, also emerged as a prevalent theme. National and local governments must work with National Sport Organizations and National Governing Bodies to develop and finance sport opportunities.

Sam and Jackson (2004) investigated the manner in which a policy paradigm affected the findings and recommendations of New Zealand's Ministerial Taskforce on Sport, Fitness, and Leisure. The goal of the taskforce was to revamp national and regional sport policy in New Zealand. To conduct this study, Sam and Jackson used qualitative methods, such as interviews, observations, and document mining, to closely monitor the actions of the taskforce.

Before describing their research, the investigators defined a theoretical framework consisting of policy paradigms which policy-makers employ to create policy. Sam and Jackson (2004) noted that paradigms are useful to analyze policy because they: (a) reflect limitations of policy, (b) convey the conundrums faced by policy-makers, and (c) relate ideological theories to field-specific ideas. Past paradigm studies revealed tension between elite sport and physical education (Houlihan \& White, 2002), the shaping and constraining role of socially constructed meanings in sport policy discussion (Chalip, 
1995; Chalip, Johnson, \& Stachure, 1996), and similarities between other national taskforces which look at their countries’ sport policies.

The researchers observed 10 of the 12 New Zealand taskforce members in daylong workshops discussing sport policy with regional decision-makers. They also read external submissions received by the taskforce and interviewed the taskforce members on several occasions. Upon conducting this research, Sam and Jackson (2004) called the taskforce’s operation a "rationalist paradigm” in which managers were concerned with organization mergers, financial cutbacks to government pay-outs, and central authority structures.

Conclusions made by the taskforce included merging sporting groups and reducing the number of sports sponsored through government grants to streamline the sport policy process. A main goal of the taskforce was to establish a "national vision” in sport development, meaning more equal treatment of sports. However, as Sam and Jackson (2004) observed, this created a difficult paradox because sport organizations were asked to seek more private sponsorships and to essentially fend for themselves more, while at the same time act more cohesively and cooperatively with other sports organizations in order to fit the "national vision”. This mandate, the researchers suggested, would result in more fragmentation among national sports organizations (NSOs).

Slack, Berett, and Mistry (1994) examined the impact of the introduction of a rational planning program on a Canadian NSO. The researchers outlined the process of rational, formal, and systematic planning for organizational restructuring. Then, through a case analysis, they demonstrated organizational conflict arising from rational planning. 
The authors did not specify the exact number of interviews conducted in their research, but used interviews of key NSO members and volunteer officials to collect data. They also mined key documents outlining Canadian sport policy produced by the federal government.

The NSO investigated by the authors was a small organization, with just 200 registered participants and only one international-caliber competition facility in the country. The organization's headquarters were located in a medium size city, near the geographic center of Canada. The organization experienced some international success in the late 1970s and early 1980s. Toward the mid to late 1980s, the federal government began to implement a rational policy for the distribution of funds for sport. In order to receive funds, NSOs needed to demonstrate conformity to certain federal regulations. In the case of the organization in the study, the government made funds dependent on moving the NSO headquarters to Ottawa, where many other NSOs were based, building a new competition facility, and restructuring the staff, which included hiring professional administrators and coaches. Previous to these demands, this particular NSO was managed almost entirely by volunteers, with only one paid staff member.

The change in organizational structure, brought about by rational policy development, created more differentiation, or sub-units (such as administrators, professional coaches) within the organization, which led to conflict. Formerly, a group of volunteers performed all the duties of the organization, but under the restructuring, professional staff took care of very specific aspects of the NSO. With more differentiation came more interdependence upon different sub-units within the organization. The researchers defined three categories of interdependence (a) pooled, 
where sub-units operate independently but all contribute to a single operation; (b) sequential, where the outputs of a first sub-unit provide the inputs for another; and (c) reciprocal, where sub-units share duties and communication. The sport organization in Slack, Berrett, and Mistry’s (1994) study could best be described as the highest level of interdependence; reciprocal. While the ultimate goal of rational planning is to create a more efficient and congruent organization, in this case, rational planning led to conflict between the previous volunteers and the new administrators. The researchers also noted the volunteers did not accept change well, adding to the conflict. They concluded rational planning is as much a political activity as a technical one and much of the conflict in the analysis stemmed from the very changes that were intended by the planning process.

Elling, De Knop, and Knoppers (2001) examined social integration through the context of sport in the Netherlands. In their comparative analysis, the researchers distinguished three dimensions of integration, namely (a) structural, (b) social-cultural, and (c) social-affective, and demonstrated how each level manifested itself within and through sport. Through an extensive literature review, they examined more closely how these dimensions of integration specifically affect four different social minority groups (ethnic minorities, physically challenged, elderly, and gays and lesbians) and the meanings associated with sport integration through these groups. Their study showed both benefits and drawbacks to integrative policy in sport organizations and examined whether government policy should encourage more integration through sport.

The authors began by depicting institutionalized levels of competitive segregation in sport, through things such as age levels, physical ability, and elite sport versus sport for all. Next, the researchers defined their three dimensions of social integration. Structural 
integration "refers to the extent to which a specific social group or person participates in required and voluntary activities in society compared with the majority of the population, especially in relation to work and education” (Elling, De Knop, \& Knoppers, 2001, p. 417). The researchers further dissected structural integration into five levels: (a) minimal, (b) competitive, (c) organizational, (d) direct, and (e) inverse. Social-cultural integration refers to the process of either confirming or challenging both dominant and marginal societal norms and values as a whole. Social affective integration refers to the ability of a process to bring different social groups together, allowing participants a feeling of inclusion and level of comfort. The researchers pointed out several contradictions in the social integration through sport concept. For example, sport can bring different social groups together through competition and camaraderie, but at the same time, can reinforce stereotypes and encourage some people to remain only in their familiar social circle.

The later portion of this article delved into current research regarding the social integration of the four social minority groups listed. In regards to ethnic minorities, the researchers stated current research was distributive in nature and current policy does not encourage organized sport segregation. However, they did point out Dutch sport organization were not free of discrimination, and for some, such as immigrants, a comfort level existed for somewhat segregated sporting groups. With regard to physically challenged people, integration was "mainly minimal or organizational in nature due to the prevailing structure and culture of competitive sport” (Elling, De Knop, \& Knoppers, 2001 p. 423). However, Elling et al. (2001) did point out advances in inverse or reverse integration in sport for people with disabilities, where the number of able-bodied participants joining sport organizations for people with disabilities was increasing. For 
the elderly, participation in sport was more social than competitive in nature. Fewer elderly people participated in sports for several reasons, such as belief in reinforced media depictions of sport for younger people, and the lack of participation as youth. The researchers noted, however, an overrepresentation of elderly, able-bodied, white males in leadership positions in sport organizations, particularly when compared to ethnic minorities and people with disabilities.

Finally, the authors noted gay and lesbian participation in sport was the least researched of any minority group and likely contained the most contradiction. Depictions of gay sports organizations and the "coming out” of elite gay athletes was shown to encourage some while discouraging others. Some sport organizations were becoming more open about the issue, but Dutch sport certainly was riddled with homophobia. The researchers concluded social integration through sport is a complex matter. Policy makers and sport managers need to be careful to examine several issues when determining social integration policy for sport.

Green (2004) analyzed two national governments’ use of “planning dictates” in relationship to developing national elite sport improvement programs in Canada and the United Kingdom. Green defined a "planning dictate” as an order or principle that must be obeyed. The Canadian government oversaw two such planning dictates, the Quadrennial Planning Process in the 1980s and the Sport Funding and Accountability Framework in the mid 1990s. These, and others implemented over the past 20 to 30 years were designed to create a process for developing elite level competitive athletes. The UK, Green asserted, was doing something similar with its Sporting Future for All and Sport: Raising 
the Game documents, and its allocation of national lottery money towards elite sport development, although the UK’s initiatives were more recent compared to Canada.

To conduct his research, Green (2004) used qualitative means, interviewing key personnel in three national sporting organizations (swimming, athletics, and sailing) in both Canada and the UK. In addition, he interviewed senior officials with Sport Canada and UK Sport, as well as other sport-policy analysts and academics. Before elaborating on the data, Green gave a detailed background of the critical realism paradigm he used to deconstruct his interviews. He took a critical theorist approach, explaining the voices of these officials are rarely heard. Sport body officials felt government policy regarding elite sport rarely took into account their opinions.

Green (2004) stated in Canada, the federal sport agency, Sport Canada, ultimately allocated federal funding for National Sports Organizations (NSOs), typically the primary source of funding for those organizations. As a result, pressure to produce successes (medals) at elite sport competition is high, and NSOs failing to produce may lose funding. In addition, sport at the grass roots level was under-funded and such organizations were left to fend for themselves. Voices from the NSOs did not agree with this policy, but understood it was how the game was played, according to Green’s interviews.

In the United Kingdom, Green (2004) asserted England is moving in a similar direction, with strong government emphasis, and in turn, financial backing, for NSOs producing high medal counts and success at the elite level. Representatives from swimming and athletics gave data to support this notion. However, the researcher did get a different opinion from a representative from sailing, who said his organization was able 
to work closely with UK Sport to develop a policy in line with the Royal Yachting Association's goals. For the most part, though, Green showed funding went towards elite level sport. Because this was a recent phenomenon in the UK, the investigator called for more research to determine whether this sort of policy will negatively impact the UK in areas of sport participation at the grass roots level or with physical education activities in schools.

Stahl, Rutten, Nutbeam, and Kannas (2002) investigated the differences in, and relationships among, policy orientation, the objective and perceived physical environment, and physical activity between Finland, Eastern Germany, and Western Germany. An earlier study by Stahl et al. (2001) found a relationship between physical environmental factors, policy factors, and physical activity status, but study results also showed the relationship weakened when “country” was controlled for. Another study by Harvey, Beamish, and Defrance (1993) demonstrated the need for researchers to consider historical constructions when examining sport and physical activity policies across nations. Therefore, the researchers in this study took historical background information into account in their investigation.

The researchers chose to do a multi-nation survey comparing the opportunities and activity habits of three regions in Europe (Saxony, Eastern Germany; NorthrhineWesfalia, Western Germany; and Pirkanmaa, Finland) which all had unique political and socio-cultural history. The study included quantitative statistical data regarding sport facilities and national investment in sport and physical activity, as well as empirical data collected from phone surveys. The first measures were taken from Finland's national sports facility database, and from the state ministries of Northrhine-Westfalia and 
Saxony. The phone survey, part of the Methodology for the Analysis of Rationality and Effectiveness of Prevention and Health Promotion Strategies (MAREPS) study, included responses from Eastern Germany $(n=913)$, Western Germany $(n=489)$, and Finland ( $n$ $=400$ ), which were cross-tabulated. Statistically significant two-way cross-tabulations were calculated through a chi-square test. Finns were the most active, while East Germans reported the most vigorous activity and were much less satisfied with available opportunities for activity. According to the facility data, Finland had a much better sport facility per capita ratio than either West or East Germany.

The researchers suggested further investigation while using their study as a blue print or pilot study. However, the article did appear to offer enough evidence to begin to support the claim of a relationship between policy orientation (political history), physical environment, and physical activity. Further study is necessary to justify these claims.

Green and Oakley (2001) studied trends present in selected Western nations to develop elite sport in their respective countries. They primarily examined whether elite sport models are becoming more uniform (diminishing contrasts) or more diverse (increasing variety) in their structure at the national level. The researchers used qualitative means to conduct this study. They interviewed key elite sport officials from certain countries (France, Spain, United Kingdom) and reviewed numerous secondary sources, such as the Elite Sport Funding Review (Cunningham, 2001), A Sporting Future for All (2000), developed by England's Department for Culture, Media and Sport and Sporting Lives: A Vision for Sport in the UK (Godfrey \& Holtham, 1999), produced by the Institute for Public Policy Research in Great Britain. 
The researchers identified key aspects to sporting success including the systematic identification of athletes, methods of coaching and training, efficiency of the sport organization, and of the depth of knowledge of sport medicine and sport science. European Eastern bloc countries such as the Soviet Union and the German Democratic Republic (GDR) made advances in these areas between 1952 and 1988, enabling athletic success but also opening the door for criticism for their use of steroids, inappropriate allocation of funds, and development of sport at the elite level while neglecting sport at the grassroots level.

Green and Oakley (2001) then examined several current Western countries' elite sporting programs, developing ten common themes: (a) clear understanding of role of different agencies involved and effective communication network, (b) simplicity of administration through common sporting and political boundaries, (c) effective system for the statistical identification and monitoring of athletes, (d) provision of sport services to create an excellence culture, (e) well-structured competitive programs with international exposure, (f) well-developed specific facilities, (g) targeting of resources for sports that have a chance to succeed, (h) comprehensive planning for sports needs, (i) recognition that excellence costs and appropriate funding, and (j) lifestyle support and preparation for life after sport. The researchers found a "flow" existed between earlier Eastern Bloc elite sport systems and current systems, pointing towards a more uniform system. However, each country's political and governmental policies lend to some individual diversity among sporting bodies.

Henry and Nassis (1999) examined the influence of clientelism in determining government funding of sporting bodies in Greece from the years 1981 to 1993. They 
defined the idea of clientelism as "the disbursement of financial, employment, or other forms of patronage in exchange for political support” (p. 43). The investigators began their study by outlining the political and historical context of Greek government during the time period specified. The researchers used qualitative means in their study, interviewing 11 high-level officials from Greek sports National Governing Bodies (NGBs) as well as eight government officials and ministers who oversaw aspects of sport governance between 1981 and 1993. In addition, the researchers used document mining to confirm data, examining party policy documents and records of parliamentary debates.

Participants mentioned several times in interviews that clientelism played a significant role in the allocation of public funds for NGBs. Several people interviewed confirmed the political affiliation of sporting bodies' boards of directors determined which sports would receive the most financing. Examination of documents such as the Ministry of National Economy State Budgets and the Department of Competitive Sport subsidy allocation backed up the interview data, showing sports affiliated with the PanHellenic Socialist Movement (PASOK) receive greater funding when that party was in power, while sports affiliated with the New Democracy (ND) received greater funding when that party was in power. The researchers concluded when it comes to governmental sports financing, clientelism exists and will likely continue to exist in Greece. The reasons for this stem mainly from a lack of trust of other political parties and individuals While not a comprehensive summary of all available national sport policy literature, the previous sample of articles demonstrates the range of factors affecting sport policy decisions in many nations. Social context, history, and politics, among others, all influence national sport policy, which ultimately affects how athletes become involved 
and participate in sport. Such a variety of national sport policies suggests athletes competing in one country may hold different viewpoints regarding the benefits and purpose of sport than those from other nations. Athletes from various countries likely perceive sport opportunities differently depending upon their background. Thus it can be reasoned international student-athletes will have different experiences and perspectives while competing at United States universities as compared to domestic student-athletes.

\section{Club Structures}

For many international student-athletes, their first experience of combining sport and academics starts at college in the United States. Prior to their sojourn, most international student-athletes participated in sports through the club system, which is a key component of many countries’ national sport policy. Many United States-born college student-athletes, meanwhile, developed their interest and talent for sport through participation in school teams (Rubingh \& Broeke, 1998). This difference between a clubbased system and a school-based one is important to understand when examining the adjustments of international student-athletes. Therefore, the ensuing studies depict the club sport structure in more detail.

Many sport clubs are voluntary associations, which attempt to organize common sport interests by users in a single venue or place. The clubs become a home for sport participation and involvement at all levels, from the beginner to the elite athlete. Because clubs are often managed by volunteers and cater to such a wide-range of abilities, the club provides a different athletic experience for future international student-athletes when compared to the United States school-based system (Rubingh \& Broeke, 1998). The clubs are also the most visible connection between sport policy and sport participation in many 
countries outside the United States. Sport clubs provide outlets for people to participate and compete in physical activity and sport. This literature review examined what makes sport clubs effective and inclusive. It also investigated where clubs fit in sport policy and how they can be most effectively governed to create opportunities which meet sport policy goals, including sport for all and elite sport development.

Koski (1995) conducted a study to assess the effectiveness of sports clubs in Finland. A five-level theoretical model defined organizational effectiveness of a sports club. The five levels consisted of: (a) ability to obtain resources, (b) internal atmosphere, (c) efficiency of throughput process, (d) realization of aims, and (e) general level of activity. Participants for the study included managerial personnel of Finnish sports clubs, contacted through the use of a national postal questionnaire $(N=835)$. Approximately half (51\%) of the questionnaires were returned, but an analysis of the surveys not returned revealed many of them to be from managers at inactive clubs.

Koski (1995) ranked clubs according to 16 variables of effectiveness which fell under the five categories listed above, then compared them using Spearman correlations. The researcher also investigated the range of effectiveness using three categories: (a) environmental variables, (b) structural variables, and (c) ideological variables. The results of the Spearman correlation revealed a strong positive correlation $(r=.60)$ between the success of a club and how widely known that club was. Another variable, ability to obtain income, also correlated positively $(r=.30)$ with how well-known the club was. Koski performed an ANOVA using the three range of effectiveness variables as independent variables, and the Pearson correlation scores as the dependent variable. Significant differences in several of the effectiveness categories existed between big city, mid-size 
city, and rural clubs. In general, participation-oriented clubs were less effective than other clubs, while clubs with clear values were more effective, particularly in ability to obtain resources and in general activity level.

Hartmann-Tews (1999) analyzed the role of sports clubs and federations in implementing the concept of "Sport for All" and how they affected structural change in sport systems using case studies from Germany and Great Britain. The researcher began by taking a general historical look at the development of sport policy in Western culture. In the late $18^{\text {th }}$ century and early $19^{\text {th }}$ century, physical activity was viewed as a means to develop a strong military. Governments also saw sport participation as a diversion for youth to keep them out of trouble. The achievement concept eventually took over sport participation, with success at elite competition driving most organized activity. Only in the second half of the $20^{\text {th }}$ century has sport participation in Western nations been seen as promoting healthy lifestyle through physical fitness, enjoyment, and well-being. These values have grown into a concept of "Sport for All"- the idea of all-inclusive sport with high participation rates for all different demographics as a goal.

Hartmann-Tews (1999) then took a closer look at the history of sport policy by focusing specifically on Germany and Great Britain. To examine current participation rates, the researcher examined two areas of involvement: (a) self-reported participation rates and (b) sport club membership numbers. Through document mining of items such as Britain's General Household Survey and the volunteer membership numbers of sport clubs in the two countries, Hartmann-Tews concluded sport participation increased significantly in the 1980s and 1990s in both countries. Much of this increase could be attributed to different groups becoming more active in sport, namely women, the elderly, 
and people with disabilities. While participation rates as a whole were increasing, they were doing so at a much higher rate in Germany than in the United Kingdom. For example, approximately 30\% of Germany’s population belonged to a sport club, compared to $12 \%$ in the UK. The researcher attributed this higher participation level to historical differences in sport policy development between the two countries.

Hartmann-Tews (1999) stated current German sport policy stems from the foundation of the German Sports Federation (DSB) in 1950, which developed after the mistrust of government organized interest groups following World War II. The DSB represents two central groups, sport National Governing Bodies (NGBs) and sport confederations. The NGBs developed and governed sport at the competitive and elite level, while the confederations "devote more attention to the promotion of recreational physical activities for all” (Hartman-Tews, 1999, p. 151). The DSB and its subsidiaries are tax-exempt because of their charitable status and they receive funding from national lottery profits.

Meanwhile, British sport policy was much more haphazard. The main governing body is the Central Council of Physical Recreation (CCPR), which was founded in 1935. In addition, a "quasi-non-governmental body" called the Sports Council was adopted in 1972 by Royal Charter. The Sports Council is funded by the government, but is an independent body, charged with fostering the "attainment of high performance standards in conjunction with the governing bodies or sport and physical recreation” (HartmannTews, 1999, p. 153). The researcher stated a lack of communication and direction between these two English bodies resulted in poor direction and leadership in the "Sport for All” movement, explaining lower participation rates compared to Germany. 
German sports policy emphasized “Sport for All” through the DSB’s promotional campaigns, while the CCPR spent less effort on grass roots level sport, instead spending more time with elite level sport. Hartmann-Tews (1999) concluded the emphasis on elite sport in Great Britain was due to the "achievement sport” concept favored by the mass media and because government funded it to a greater level than "Sport for All" initiatives.

Horch (1994) examined the relationship between German sport clubs receiving government funding and their perception of autonomy to govern themselves. In Germany, most sporting activity takes place in sports clubs, which often depend upon federal, state, and local financial assistance to exist. The researcher suggested the "larger the share of government resources in the budget of a voluntary association, the greater the loss of autonomy” (p. 270). To set up his study, Horch examined several theoretical frameworks, including resource-dependency-theory and social-exchange-theory in an attempt to explain the relationship between the clubs and their government funders. Horch elaborated on his theory, including several other factors influencing an organization's ability for autonomy, from the view point of the organization. These factors included: (a) the number of resources or donors contributing to the organization, (b) potential alternatives for the association, (c) number of cooperative relations of the association, (d) benefits of government resources for the association, (e) the cost of the financing for the association, and (f) the counter influence of the association. In addition, the researcher examined three other variables affecting the relationship, looking at them from the perspective of the government or donor. These were (a) alternatives to the 
association for the government, (b) the benefits of financing for the government, and (c) cost of financing of the government.

The investigator surveyed leaders of 397 registered associations located in the Hamburg area of Germany, 105 of them sport clubs. Association leaders were asked subjective questions and five objective questions, rated on a 4-point Likert type scale, to determine the club's level of autonomy. Meanwhile, each association’s budget was examined to determine the percentage of funds coming from government sources. Sport clubs had the lowest budget percentage of the five associations studied, although Horch (1999) added most sports clubs receive financing from National Sport Organizations (NSOs), which are, in turn, funded by the government. Thus NSOs act as a buffer, further limiting the influence of government on the clubs' operations. Correlations were run with the percentage of the association's budget coming from government sources and the level of autonomy felt by the directors. For sport clubs, the correlations were low both in subjective questioning and through objective analysis. Horch concluded the strongest correlations were for associations not producing public goods, which was not the case for sport clubs as they produce a public good in the form of teams and activities.

Looking specifically at sport clubs, the researcher ran a trivariate regression analysis, which again included the dependent variable of perception of autonomy, and two independent variables: percentage of budget from government funds and an item from the list of factors mentioned above (e.g. number of resource donors, other alternatives, cost of financing association). One significant finding was the number of governmental levels involved in financing the club's budget was the biggest indicator of loss of autonomy $(\beta=.57)$. 
He also ran a multiple regression analysis with perception of autonomy as the dependent variable and the factors listed above as the independent variables. Four variables stood out as significant in predicting a total of $40 \%$ of the variance in governmental influence on the association. Influence was greater as the number of donors went up, as the size of the association went up, as more alternative choices for the government were available, and the less the goals of government overlapped with those of the association.

In conclusion, Horch (1994) noted loss of autonomy was not as important for sports clubs as it was for other associations. He also stated the most important factors with government donation were the perceptions of the donor (government), not the association. Government could influence the associations much more than it did. Horsch attributed this lack of influence to government benefits not being great enough and a lack of desire on the government to exert influence.

Ulseth (2004) examined the differences in social integration patterns of Norwegian users of voluntary sports clubs compared to those of for-profit fitness centers. Traditionally, volunteer sport clubs in Norway, as in many other nations, exist to allow participants opportunities to play sport, but also for social interaction. Voluntary sport club members typically pay an association fee, but also work at voluntary activities, while additional funding for the club may come from sponsorships, government grants, and outside rental of the facility. Since the 1990s, fitness centers have grown in popularity in Norway. The goal of these centers is to make a profit, and members typically pay a higher membership fee, but are not required to make any volunteer commitment to the organization. 
Ulseth (2004) used the information from two postal surveys, the Sports Club Study $2000(N=1,205)$ and the Fitness Centre Study $2001(N=1,585)$, to examine social behaviors in the club and fitness centre settings. The club study included randomly selected participants who were older than 12 and were members of 549 different sports clubs in Norway, while the fitness centre survey was sent to a randomly selected population of 4,099 members of three major Norwegian fitness centre chains. The researcher used both simple descriptive statistics (frequency analysis) and linear regression to analyze the data. In the regression, the independent variables included gender (two levels), place of residence (two levels: city or outside city), age (four levels), martial status (two levels), education (unclear), income (three levels: low, medium, high), and chief occupation (six levels: work, school, domestic work, unemployed, pensioner, and social security). Dependent variables included: (a) whether new friends were made through exercise, (b) how often one met 'fellow exercisers' outside the venue, and (c) whether the physical or the social part of the exercise was considered most important by the exerciser. A regression was run using the question "do you gain new friends through exercising at the fitness centre or in the sports club?” For fitness centre respondents, gender, age (18-25 and 56-older), and chief occupation (pensioner) yielded the only significant results, while in sports club respondents, only gender and income level (high) proved significant. All independent variables in the regression accounted for less than $7 \%$ $\left(r^{2}=.066-\right.$ fitness centre, $r^{2}=.046-$ sports clubs) of the variance. A second regression was run, using venue (fitness centre or sports club) as an independent variable, causing the $r^{2}$ values to jump to over $50 \%$. 
Simple frequency analysis of the question of making new friends revealed $81 \%$ of the sports clubs participants said yes, compared to just $14 \%$ of fitness centre respondents. To the question of joining other members outside the exercise setting, 53\% of the fitness center members said "almost never," much higher than the $19 \%$ of club members who said yes. However, $14 \%$ of fitness center members said yes to meeting every day, $7 \%$ higher than club members. At all other levels, namely (a) twice a year, (b) once a month, (c) once a week, and (d) several times a week, club members said yes at a higher rate than fitness centre members.

Finally, respondents were asked whether they exercise in their venue for the social aspect or physical aspect by completing questions on a 7-point Likert-type scale, with anchors of "the social part" (1) and "the physical part" (7). Fifty-eight percent of club members marked either 1 or 2 , while only $2 \%$ of fitness centre members responded 1 or 2 . At the other end, $10 \%$ of club members marked either 6 or 7 , while $84 \%$ of fitness centre members marked 6 or 7 . Ulseth (2004) concluded the two venues contributed to social integration, but in distinct ways. Sport clubs were more likely to produce new friendships and those friendships were more likely to exist outside of the club, compared to fitness centers. However, fitness centers had an important contribution as places for friends to meet and places to maintain already established friendships. With the rising popularity of fitness centers, Ulseth concluded the social interaction and integration aspect of exercise venues was changing.

The preceding article reviews examined different aspects of the club sport system employed in most nations outside the United States. This research demonstrated clubs can be effective at fostering the concept of "Sport for All" and providing a positive social 
outlet, in addition to recreation venue, for people of all ages. The aspects of sport which clubs emphasize, however, is often impacted by funding and influence of government. Understanding the background of club-based sport systems is important in understanding the experience of international student-athletes who typically grow up in the club sport system.

International Youth Sport Participation Trends and Youth in Sports Clubs

While several studies have documented the impact of sport club effectiveness, and autonomy, such studies typically focus on the adult participant. Most sport clubs are financed through membership fees and sponsorships, often stemming from adult patrons. However, as the current study examines the experience of the international studentathlete, it is important to understand how sport clubs affect youth participants. Clubs typically offer youth important opportunities. Siedentop (1995) defined three main goals for youth sport participation: (a) educative, (b) public health, and (c) elite sport development. This literature review examines how clubs work at the youth level to meet these goals and what happens to levels of sport participation as youth progress through the club structure. It also examines the role of coaches, parents, and administrators in promoting youth sport participation.

MacPhail, Gorely, and Kirk (2003) investigated the early socialization of English children into a sports club. The researchers chose qualitative means for their study, collecting data through an 18-month ethnography at a youth sports club. Primary data collection methods included observations and field notes, group and individual interviews, and a psychometric survey. The researchers interviewed 47 children between the ages of nine and 15, all members of an athletics club, which was given the fictional 
name Forest Athletics Club (FAC) in this study. They interviewed five coaches and the club chair, as well as eight parents. They also observed training sessions and competitions, as well as club meetings, typically during Monday and Wednesday sessions, between September 1999 and December 2000. In order to better triangulate the data, an additional set of youth $(n=51)$ were administered a survey to gauge measures of enjoyment, motivation, achievement goal orientation, and perceived motivational climate. The survey measured enjoyment using a subscale from the Intrinsic Motivation Inventory (McAuley, Duncan, \& Tammen, 1989), and intrinsic motivation using a scale from the Perceived Locus of Causality (PLOC; Goudas, Biddle, \& Fox, 1994). Achievement goal orientation was measured using a scale from the Task and Ego Orientation in Sport Questionnaire (TEOSQ; Duda \& Whitehead, 1998) and perceived motivational climate was measured using a scale from the Perceived Motivational Climate in Sport Questionnaire Version 2 (PMCSQ; Newton. Duda, and Yin, 2000).

MacPhail, Gorely, and Kirk (2003) based much of their study off the work of Siedentop (1995) who argued three main goals of youth sport participation: (a) educative, (b) public health, and (c) elite sport development. The literature points to an emphasis by most national sport policy makers, through both written policy and allocation of funds, toward elite sport development, with little attention paid to Siedentop’s first two factors. MacPhail et al. (2003) also cited the work of Cote and Hay (2002), which demonstrated a three-step pattern for the socialization process of youth involved in sport: (a) sampling, (b) specializing, and (c) investing. The investigators focused this study on the sampling phase. They found key characteristics of this phase to be: (a) involvement in a range of sports and other activities, (b) experiencing simple fun and enjoyment in their sport, (c) 
competition, (d) fitness and health benefits, (e) deliberate play, and (f) friendship and peer relations.

Evidence for involvement in a range of sports and activities derived from interview answers. Many children simply told the investigators the wide range of activities in which they were currently involved, with club athletics only being one such item. Fun and enjoyment was documented by both interview answers as well as responses on the PLOC which demonstrated high intrinsic motivation scores (mean RAI = 14.88, $\mathrm{SD}=$ 9.86). The competition factor focused much more on skill mastery for younger participants (ages 9-11), while the older segment viewed winning and losing as more important. Answers to the PMCSQ revealed the club climate was perceived to be much more task (skill development) oriented, than achievement oriented. Boys (mean ego score $=37.32$ ) had significantly higher ego scores than girls (mean ego score $=29.76 ; F(2,43)$ $=5.47, \mathrm{p}<0.05$ ), suggesting boys felt the climate was more geared towards competition compared to girls.

Kirk and MacPhail (2003) examined the construction and make-up of a youth sports club through the concept of social positioning. The researchers used qualitative means, choosing a year-long ethnography to explore the structure and relationships within a British youth track and running club. The researchers selected three different groups for their study: (a) youth participants, (b) coaches, and (c) parents. To generate their data, Kirk and MacPhail observed and took field notes and conducted individual and group interviews. One researcher became rather active in the club structure, which aided in data collection. Group interviews were carried out with 47 children between 9 and 15 . 
In addition, individual interviews were conducted with four coaches and the club coaching coordinator, and with nine parents of regular attenders.

Social positioning is a concept defined as a structuring property of a society, where different positions cross time and space (Giddens, 1984). These positions create different meanings from various viewpoints and are not static. In this study, the researchers examined how different social members of the sports club viewed each other and themselves to function as a single, effective club.

During and after data collection, the researchers employed constant comparative analysis techniques. Youth participants were classified into two groups: (a) samplers consisting of beginners with more emphasis on play than on disciplined training and competition, and (b) beginning specializers - consisting of older, more experienced youth who had deliberate practice and play intentions. The samplers were often involved in many other activities outside the club and found sport to be a means of fitness, friendship, and fun. They were not as interested or concerned with high level, organized competitions. The beginning samplers, on the other hand, were involved with fewer activities outside of the club because they wanted to concentrate on improving in track and running. They attended practice sessions regularly, while competition and winning were more important goals.

Parents were classified into four different positions: (a) non-attenders, (b) spectators, (c) helpers, and (d) committed members. Non-attenders appreciated having their children involved in sport, but typically only dropped them off and picked them up because of work commitments or because they felt their child did not want them there. Spectators enjoyed watching their children, but did not get involved at all with the club. 
Helpers, on the other hand, did get involved with the club, either assisting the coaches or volunteering to help with meets or run the concession stand. Helpers typically started as spectators, but once they felt comfortable with the club and saw how the club functioned, were willing to volunteer their time to keep the club running. Only one parent fell into the category of committed member. He was a father who felt his daughters received great benefit and enjoyment from the club and wanted to continue that experience so he became a committee member within the club structure.

Kirk and MacPhail (2003) noted coaches felt a particular importance in their role of committed volunteer. The coaches were in the same social position, despite being critical of one another, because of the way they viewed their contribution to the club, the role of parents, and the importance of the club for children. The researchers felt this belief existed because the coaches were working most frequently with beginners and samplers. The coaches placed great importance on the benefits gained by the children, teaching them respect and committed behavior towards their athletic endeavors. On the other hand, the coaches constructed positions for parents, which were not often met. The coaches viewed several of the spectator parents as freeloaders, who were not as committed to the club as the coaches themselves were. Ultimately, the coaches felt a position of privilege because they saw themselves as committed volunteers and saw it as their right to be critical of parents.

The authors concluded there were several understandings of the club structure. Several different agendas existed based on social position. The goals of the samplers differed from those of beginning specializers, sometimes creating conflict within practices. The same was true among different groups of parents or between committed 
volunteer coaches and spectator parents. Understanding social positioning, which includes seeing others' viewpoints, would likely lead to a better understanding of sport clubs, and would assist administrators in creating more efficient operations.

Collins and Buller (2000) studied the outcomes of the Nottinghamshire Sports Training Program (NSTP), investigating the levels of satisfaction and intention to continue sport participation by youth who had gone through the program. The researchers used a mixed-methods case study approach, sending out postal questionnaires to 898 past participants and their parents, and interviewing several club coaches and officials. Of past participants, 352 responded $(n=352)$ for a 39\% return rate, while 310 parents responded $(n=310)$, a 35\% return rate. Participant responses were split nearly evenly by gender (51\% male, 49\% female). In-depth interviews were conducted with the nine coaches of the sports examined in the study (basketball, cricket, hockey, orienteering, netball, rugby, squash, table tennis, and tennis), and telephone interviews were conducted with coaches or club officials from other clubs to assess the 'youth friendly' practices of those clubs.

The NSTP stemmed from part of a national initiative in England to create better sporting opportunities for all children, regardless of their home location or economic status. The national scheme, called Champion Coaching, was founded in 1991 after the Minister of Sport, Robert Atkins, earmarked 700,000 pounds to go towards the National Coaching Foundation, which established programs such as the NSTP. Through a literature review, the researchers outlined four levels of influence of youth sport participation: (a) school, (b) local area, (c) television and media, (d) other significant people. Collins and Buller (2000) examined whether the NSTP was effective in overcoming obstacles among these four levels, and whether participants, upon 
completion of the NSTP, were more likely to continue sport participation and were aware of their opportunities to do so.

The researchers used descriptive statistics and participant/parent comments for their analysis. They found 96\% of participants enjoyed the NSTP course. Surveys included a question asking what aspect of the program was most enjoyable, with seven possible responses: (a) coaching, (b) new skills, (c) games, (d) equipment, (e) facilities, (f) making new friends, and (g) competition. 'Games' was the most enjoyable factor (27\%), followed by 'learning new skills' and 'coaching' (both 23\%). Girls found 'games' and 'meeting new friends' more appealing than boys (26\% to $16 \%$ and $15 \%$ to $7 \%$ ), and boys enjoyed competition more than girls (14\% to 9\%). Participants also noted high satisfaction with venue accessibility and location, as well as the level of coaching. However, a major finding was just a $60 \%$ positive response to the question of whether participants received adequate information about where they could continue their sport participation ('exit routes') after the NSTP was complete. The researchers also noted only $25 \%$ of respondents joined a club after completion of the program, while $46 \%$ continued participation in their sport just on a recreational basis.

The researchers also compared the response data to "social need" demographics in Nottinghamshire. To perform this comparison, Collins and Buller (2000) examined postal codes of responses and compared them to the "social need" make-up of that postal code according to a 1994 report by the Nottingham, Notts Planning and Economic Development Department. The comparison revealed 87\% of the NSTP participants came from areas of "below average social need," compared to $71 \%$ of the general population. This meant in the three categories under "below average” (moderate, serious, and 
extreme social need) participants were underrepresented, $13 \%$ to $29 \%$. The investigators concluded the NSTP is a successful program in many respects, but is lacking in two key areas: the provision of 'exit route' information to participants and inclusion of participants from all levels of "social need."

Butcher, Linder, and Johns (2002) investigated the extent, context, and timing of students who withdraw from competitive youth sports in Canada. The study included students in $10^{\text {th }}$ grade from 50 classrooms in 10 different high schools of six divisions of a mid-size Canadian city. In all, the researchers surveyed 1,387 students including 721 males and 666 females, questioning them retrospectively about their sport experiences from first grade through tenth grade. Participants filled out a questionnaire, which included a section describing their sporting profile and another section which included details about their process of dropping out of sports. The researchers also collected demographic information.

The researchers used a pilot test-retest of their instrument on 22 students to assess reliability, and surveyed 11 parents as pilot test subjects. The investigators then ran a correlation between parent answers and youth answers to test for validity. The test-retest resulted in coefficients of greater than .72 for all but two items while the correlation tests were mixed, which validated some questions, while raising others.

Independent variables tested included gender and “dropout type” which was broken down into four levels: (a) sampler, (b) low level participants, (c) high level participants, and (d) elite level participants. Dependent variables included: (a) sports participated in since first grade, (b) total sports dropped since first grade, (c) years participated in each dropped sport, (d) number of simultaneous sports at withdrawal, and 
(e) grade dropped. Significant correlations occurred between gender and the first three dependent variables, as well as dropout type and all variables. The researchers also conducted an ANOVA using gender as an independent variable and 12 reasons for dropout (as listed on the survey) as dependent variables. They also ran three MANOVAs with independent variables of dropout type (three levels listed above), grade level (elementary, junior high, senior high), and program type (interschool, community club, and private club). The independent variables in the ANOVA and all three MANOVAs consisted of 12 reasons for dropping out of sport participation: (a) did not enjoy, (b) wanting more time for non-sport activities, (c) other sports took too much time, (d) not good enough, (e) too much pressure to perform well, (f) needed time for studying, (g) coaching, (h) injury, (i) no longer opportunity to play, (j) took a job, (k) too expensive, and (l) discouragement from parents. In the gender ANOVA, significant results were found with dependent variables d, e, f, h, and j. In the dropout MANOVA, significant results were found with dependent variables a, b, d, e, i, k, and l. In the grade level MANOVA significant results were found with dependent variables a, d, g, h, i, and $\mathrm{l}$ and in the program type MANOVA, significant results were found with dependent variables a, c, e, g, j, and l.

Butcher, Linder, and Johns (2002) concluded reasons for dropout were quite varied but not alarming, as almost all students (94\%) dropped out of sport participation at one point or another, yet most became active in another sport. The number one reason for dropping out was lack of enjoyment, a finding the researchers suggested needed further examination. Females noted lack of competence and pressure to perform were much more important reasons for dropping out then reasons documented by males. 
Vanreusel, Renson, Beunen, Claessens, Lefvre, Lysens, and Vanden Eynde (1997) conducted a study to determine whether youth sport participation carried over into adult sport participation in Belgium. Past studies in sport socialization examined major socializing agents, such as school, parents, and peers, however, McPherson (1986) has pointed out socialization should be viewed as interactive, not a series of outside forces affecting those studied.

In their study, Vanreusel et. al. (1997) carried out a longitudinal study of 236 boys by examining survey data collected in the Leuvern Growth Study from 1969 to 1974. Boys in the study were surveyed each year between ages 13 and 17 . The researchers then reinvestigated those same participants 17 and 22 years later when participants were 30 and 35. Amount of time spent on sport was the unit of measurement in the studies, with four categories: (a) inactive - less than one hour of activity per week, (b) moderately active -1 to 3 hours per week, (c) active -3 to 6 hours of activity per week, and (d) very active -6 hours or more of activity per week. The investigators only used subjects from the "inactive” and "very active" categories from the original Leuvern Growth Study. When subjects were surveyed in their 30s, they could only choose from three categories: (a) inactive - less than 1 hour, (b) moderately active - from 1 to 3 hours, and (c) active more than 3 hours. The young subjects also had their youth sports career classified as either competitive or recreational.

By simply comparing statistics, the researchers demonstrated youth participating in recreational sport were more likely to continue participation into adulthood, compared to youth involved in competitive sport. The researchers then went further, running interage correlations for sport participation from ages 13 to 17, and from youth ages (13 - 17) 
to 30 and 35, with several significant results. Sport participation at young ages of 13 and 14 had significant correlations with participation at 17 and 18 years-old. For example, the correlation between participation at 13 and at 18 was .37. In the second correlation, participation at 17 and 18 had a relatively high $(\mathrm{p}<.01)$ correlation with sport participation at age 30. One unusual finding was that participation at 13 had a high correlation with participation at age $35(.20 \mathrm{p}<.01)$, while participation at 14,15 , and 16 were far less correlated.

Vanreusel et. al. (1997) completed their study by categorizing all participants into nine categories. The largest percentage (37\%) of subjects went under the label "unstable sport participation in youth and in adulthood.” The second highest was "unstable sport participation in youth, inactive in adulthood” (14\%). The smallest percentage (1\%) fell under the heading “decreasing sport participation in youth, increasing sport participation in adulthood". The key finding was that active sport participation in the latter years of youth (17 and 18) strongly correlates with whether adults at 30 and 35 participate in sport.

Collins and Buller (2003) examined whether social need was a limiting factor in youth sport participation, with specific reference to the "Performance Resources" initiative used in Nottinghamshire, England. The researchers used a mixed methods approach to their study, collecting some quantitative data through a survey mailing, then following up with qualitative interviews with selected individuals. The specific number of questionnaires mailed was not given, but 539 responses, (417 male, 122 female) were returned, which was a rate of $38 \%$. The researchers conducted phone interviews with 70 
of these respondents, or $15 \%$ of the total. They also interviewed three of the coaches involved in the program.

The Performance Resources plan, which was originally called Performance and Excellence in Nottinghamshire Sport scheme (PENS) when it was established in 1994, had five objectives: (a) covering levels of the Sports Development Continuum throughout the county, (b) providing clear and accessible pathways for talented youth to advance up the sport ladder, (c) increasing the number of junior sport clubs, (d) develop effective coaches, and (e) complimenting physical education and sport activity in schools. This plan has been applauded for its success in promoting sport for youth, particularly in getting youth to continue their involvement in sport after they have gone through the program. The researchers found these statements to be true, citing $46 \%$ of their respondents becoming involved in higher levels of sport after the program. However, the researchers also examined the extent of the program's outreach and determined poorer children were much less likely to participate in the program. Collins and Buller (2003) divided their participants into four social needs categories: (a) below average, (b) moderate, (c) serious, and (d) extreme. After eliminating children involved in multiple sports, and those living outside Nottinghamshire county, they were left with 319 participants, 293 of whom fell under the 'below average’ social needs category. Only 26 participants fell under the other three groups, including just one under 'extreme' social need.

In conclusion, the researchers agreed the program was beneficial and had merit, particularly after discussing the program in the interviews with participants and coaches. 
However, they determined the program still needed to become more inclusive for youth of all economic backgrounds.

MacPhail, Kirk, and Eley (2003) examined what could be done to encourage more youth to become involved in organized sport in England and Wales. A key difference in their study, compared to many others examining youth sport participation, was their use of youth participants' suggestions for policy change, instead of using the more commonly investigated sport manager opinions.

The researchers used qualitative means in their investigation, employing a technique called Nominal Group Technique (NGT). They interviewed a total of 608 British youth, all between the ages of 14 and 18, including 279 males and 329 females. All participants were students selected by their physical education teachers to attend sport leadership workshops held in one of seven different British cities. In each workshop, participants were put into groups of 12. Researchers at each site then went through identical procedures, which included the following as prescribed by NGT: (a) introduction, (b) generation of ideas through writing, (c) round-robin feedback and recording of ideas on a flip-chart, (d) discussion of recorded items for clarification, and (e) individual voting and rating of responses. The main research question asked at each site, 'What can be done to help young people participate in sport?' Investigators used grounded theory to analyze the data collected, which included over 1,000 written responses to their research question. Statements were grouped and labeled at each of the seven locations. The authors then compared each site's results for further identification and grouping. Ultimately, the researchers developed five major categories of responses to their question: (a) people, (b) conditions, (c) resources, (d) 
climate, and (e) attractors. Each of these five categories had either three or four subcategories.

MacPhail et al. (2003) concentrated their study on the two categories with the most responses: conditions (370 related statements) and climate (222 related statements). Under climate, the subgroup 'inclusivity’ garnered the most responses (125 related statements) which led the investigators to the conclusion youth are more concerned with having more opportunity to participate and further their skills ('mastery') instead of elite competition ('performance'). Under the conditions category, the subgroup 'school and club provision’ was most prevalent (217 related statements). Students desired more physical education opportunities and more variety in school settings, which are typically dominated by a few traditional sports. Other suggestions by participants under the conditions category included a desire for stronger correlation ('coordinated pathways') between school sport and club sport and more accessibility for participation in sport clubs.

Roberts (1996) examined the British government policy document Sport: Raising the Game, comparing it with three extensive national surveys gauging youth involvement in sport. The three surveys included: (a) a 1994 participation survey by the Office of Population, Censuses and Surveys (OPCS) for the Sports Council, (b) a 1995 study by the OPCS for the Sports Council regarding school sports facilities, and (c) a 1995 study by the OPCS for the Department of Education focusing on youth involvement in service, with additional information collected regarding youth participation in sport and leisure activities. The participation survey included a national sample of 4,400 6-16 year olds, while the facility survey included data from approximately 1,000 schools, and the youth 
service survey had a national sample of 3,700 young people between the ages of 11-25. The researcher exclusively used descriptive statistics to draw his comparisons.

Roberts (1996) pointed out many inconsistencies between Sport: Raising the Game, which cited a decline in youth sport involvement, and results from the surveys, which illustrated high levels of sport involvement among English youth. For example, in the facility survey, $90 \%$ of respondents (typically physical education or head teachers) stated their school regards sport as very important and nearly half of the respondents noted increases in extra-curricular sport options in the past three years, while just 10\% reported a decline. Roberts asserted the data from the surveys pointed towards other increases in youth sport participation. He noted, however, that activities were not simply the traditional English team sports of football, rugby, and cricket, thus participation in those sports may have appeared to decline. Physical educators showed a belief in the "Sport for all” concept and have actively been trying to introduce different sport and exercise activities to increase involvement.

Roberts (1996) was also critical of the policy's lack of research regarding standards for adequate exercise and activity time for young people. Sport: Raising the Game stated British youth should spend two hours per week in formal physical education lessons, but did not back up the assertion with scientific evidence. The author went on to depict English youth as actually spending much more time in physical activity outside of school hours, a phenomenon which did not occur nearly as much in the previous decades. The youth service study also showed $47 \%$ of youth chose "going to sport and leisure centers” at least once a month and 56\% spent leisure time playing sport, a higher 
percentage than going to movies (42\%), going to youth clubs (32\%), going to pubs (11\%), and attending concerts (8\%).

The government policy stated there were lopsided gender differences in opportunity to play sport, another assertion Roberts (1996) claimed was untrue. He reasoned girls had as many opportunities, but chose to participate less and in different physical activities, such as aerobics, dance, and horse riding, than their male counterparts. Meanwhile, class differences did not appear to affect youth exposure to sports opportunities, but did limit the amount of time youth from lower economic income families could participate.

In summary, youth who grow up outside the United States have many different sport participation options, many of which start in clubs rather than schools. Governmentsupported initiatives often are used to prop up youth sport opportunities, but little is really understood about why youth make sport participation-related decisions. The decision for international university students to journey to the United States to play college sport could very well be impacted by their youth sport experiences. Thus it is important to understand the background and effectiveness of youth sport programs in countries outside the United States. The studies reviewed illustrate some of the issues currently being studied regarding youth sport participation.

\section{Purpose of Sport}

As demonstrated in the literature, many nations utilize various policies and structures to develop systems for sport development. These structures can impact how people view the purpose of sport. Several researchers have examined differences in how individuals perceive the purpose of sport, often linking the perception to goal or 
achievement orientation. Past studies outlined differences in perception of sport between male and female high school athletes (Duda, 1989), intercollegiate and recreational college athletes (White, 1995), amateur and professional soccer players (Carpenter \& Yates, 1997), and amateur and professional rugby union players (Treasure, Carpenter, \& Power, 2000). Duda and Allison (1990) suggested culture could impact psychological perceptions of sport and called for cross-cultural studies examining perceptions of sport. To date, however, no one has conducted such cross-cultural studies. The following studies illustrate research focusing on perception of sport begining with Duda and Allison’s call for cross-cultural examination.

Duda and Allison (1990) outlined the dearth in literature examining cross-cultural differences which account for psychological difference in athletes and their approach to sport. The researchers cited several studies examining race relations in team sports and issues of racial and ethnic stratification of athletes in elite sport settings. Few researchers, however, have looked at the way cultural, racial, and ethnic membership affect beliefs regarding sport and competition, despite ample evidence that such membership affects psychological and socialization patterns in many non-sport settings. The authors began their paper with a breakdown of articles published in sport psychology journals and found less than $4 \%$ of them included racial/ethnic origin as a demographic variable.

Duda and Allison (1990) noted past researchers have investigated ethnic, racial, and cultural differences in physical performance capabilities, motor skill development, and participation levels in competitive and recreational sport. All of the studies examined showed that in culturally pluralistic countries such as the United States, significant differences existed among some racial and ethnic groups, thus supporting a need for 
research into psychological differences between groups relating to sport motivation. In addition, the literature indicated when cross-cultural groups adopt the practices of their host cultures, they often view the practice in a different way, adding their own ethnic identity to the activity, which includes sport participation. Examination of cross-culture sport psychology would detect such differences. When differences are detected, the researchers suggested actions and perceptions of sport by the host culture will be better defined and sport psychologists, coaches, and others who work with cross-cultural athletes will be more sensitive and understanding of athlete needs.

Duda and Allison (1990) suggested several methodologies to help detect cultural differences in sport psychology and develop an interpretive framework for such study. Suggestions included: (a) more ethnographic qualitative studies, (b) heavier reliance on “insiders or key informants” (p. 125), (c) quantitative instruments tested for validity on cross-cultural populations, and (d) more use of culture as a key variable.

Duda (1989) examined the relationship between athletes who were psychologically task-oriented or ego-oriented and whether a relationship existed between such orientation and their perception of the purpose of sport participation. Previous literature suggested individuals define goal perspectives two ways. In the first way, called task-orientation, individuals judge their success based on self-mastery of skill and selfimprovement ability. With the second perspective, called ego-orientation, individuals judge their achievement based on their performance or ability relative to others. In simplified terms, task-oriented individuals look inwardly, while ego-oriented individuals look outwardly to assess whether they succeed or fail in various tasks and goals. 
Duda (1989) suggested in the athletic arena, individuals who are more taskoriented will view the purpose of sport as important for its ability to develop positive attributes in the participant such as good health, satisfaction in mastering a skill, and learning team work. She suggested individuals who were ego-oriented would view the purpose of sport as competition, enhancing one's social position, and winning. To conduct her investigation, Duda adapted instruments and questions from previous researchers who looked at task and ego orientation in the academic setting and developed a Purpose of Schooling Questionnaire. Duda’s Task and Ego Orientation in Sport Questionnaire TEOSQ contained 15 items, in which participants responded on a 5-point Likert-type agreement scale. Her Purpose of Sport Questionnaire was a 60-item instrument in which participants again responded to items on a 5-point Likert-type agreement scale. The instruments were given to high school athletes $(N=321)$, a sample comprised of 128 males and 193 females from six different midwestern United States high schools.

The researcher conducted factor analyses on the results from both instruments. For the TEOSQ, responses were put into two subgroups consisting of basketball players $(n=123)$ and athletes from all other sports $(n=198)$. Six different items for both subsets loaded above a .4 factor weight on two factors, called task involvement and ego involvement. Internal consistency of the factors measured by Cronbach alpha coefficients produced acceptable levels of .82 (task) and .89 (ego) in the basketball player subset and slightly lower levels of .62 (task) and .85 (ego) in the other athlete subset. With the Purpose of Sport Questionnaire, Duda (1989) ran all responses through a single factor analysis, resulting in 16 items loading over a .4 factor weight on seven factors. She 
labeled these factors: (a) mastery/cooperation, (b) physically active lifestyle, (c) good citizen, (d) competitiveness, (e) high status career opportunity, (f) enhance self-esteem, and (g) social status/getting ahead. All factors produced alpha coefficients between .75 and .83. The seven factors accounted for $81 \%$ of the variance in purpose of sport.

T-tests run on the data revealed females rated significantly higher on task orientation while males were significantly higher in ego orientation. Results of a MANOVA using gender as the independent variable revealed females saw mastery/cooperation as a more important purpose of sport, while males saw competitiveness, social status, and high status career opportunity as more important. In addition, task orientation correlated positively with master/cooperation, active physical lifestyle, good citizen and enhanced self-esteem, while correlating negatively with social status/getting ahead. Other results demonstrated achievement orientation is closely correlated with belief in the perception of sport. Purpose of sport scores explained over $28 \%$ of the variance in task orientation, which jumped to $69 \%$ for male athletes only. This research suggested the more athletes are ego-oriented, the more they see sport as a means to enhance social status and career status. The more athletes are task-oriented, the more they see the purpose of sport as improving mastery of a skill, learning cooperation, being a good citizen, and living an active lifestyle. Duda (1989) suggested her findings point not to the sport affected goal orientation, but rather what is emphasized in the teaching of the sport.

White (1995) investigated differences in how male and female varsity and recreational college athletes perceive the purposes of sport participation. Past research in education and sport demonstrated that high school aged athletes exhibited varying views 
of what should be the preferred outcomes of participating in sport. In previous studies, participants saw the purpose of sport as either enhancing materialistic and individualistic goals or developing intrinsic satisfaction and positive socialization attributes. Duda (1989) developed a Purpose of Sport Questionnaire, which singled out seven factors affecting perceptions of purpose of sport participation: (a) mastery/cooperation, (b) physically active lifestyle, (c) good citizen, (d) competitiveness, (e) high status career opportunity, (f) enhanced self-esteem, and (g) enhanced social status/getting ahead.

In her study, White (1995) administered the 46-item Purpose of Sport Questionnaire to 212 United States college students attending northeastern schools. Of that sample, 74 were male intercollegiate athletes, 59 were female intercollegiate athletes, 41 were male recreational athletes, and 38 were female recreational athletes. Participants responded to questionnaire items on a 5-point Likert type agreement scale. The same seven factors used in the Duda (1989) study were also used in this one. All factors produced satisfactory internal consistency ratings, as Cronbach alpha coefficients ranged from .79 to .87 . The researcher utilized a 2 x 2 MANOVA, using the independent variables of gender and level of sport involvement, while the dependent variables included the seven Purpose of Sport factors.

Results of the tests showed female recreational sport participants differed significantly from the other three groups. Female recreational sport participants highly emphasized sport being about fostering cooperative skills, enhancing personal mastery, promoting good citizenship, and helping lead a physically active lifestyle. Male intercollegiate athletes differed significantly from the other three groups in the way they placed high emphasis on sport enhancing career and social status. When comparing just 
gender, White (1995) found males felt sport participation should lead to improved career opportunities, higher social status, and should make an individual more competitive, significantly more so than females. Intercollegiate sport participants placed a significantly higher emphasis on competitiveness and high-status career development than did recreational participants. Meanwhile, recreational sport participants placed significantly more emphasis on the categories of physically active lifestyle and good citizenship.

White’s (1995) findings suggested collegiate females were more concerned with sport being the end in itself, while collegiate males were more likely to find elite sport participation as a means to an end, namely the rewards of high career and social status. Recreational participants also tended to view sport's purpose as intrinsic and inwardly rewarding, while intercollegiate athletes were more motivated by external rewards.

Carpenter and Yates (1997) examined the relationship between personal goal perspectives and perceived purpose of sport participation among amateur and semiprofessional English soccer players. Past research (Duda, 1989) indicated the goal perspectives of athletes are either task-oriented or ego-oriented. Task-oriented athletes saw the value of sport as an end in itself, teaching self-discipline and mastery of skills, as well as developing personal health and well-being, things measured by self performance. Ego-oriented athletes saw sport as a means to an end, a way to gain other things such as recognition, status, and non-sport opportunities. Athletes with an ego-oriented goal perspective measure their achievement as compared to others.

Duda (1989) was able to connect this two-prong goal perspective with specific athlete traits by developing the Purpose of Sport Questionnaire and utilizing the 
instrument with male and female high school athletes. Carpenter and Yates (1997) used very similar methodology, but were the first to use a sample of elite and non-elite athletes in the same sport. In their study, the researchers surveyed 132 British male soccer players which included 66 semiprofessional players who were playing in a feeder league to a top English pro league and 66 amateur players who were playing in a competitive local league. Participants completed two instruments, the 13-item Task and Ego Orientation in Sport Questionnaire (TEOSQ; Duda and Nicholls, 1992) and a 21-item Perceived Purposes of Soccer Questionnaire (PPSQ), which was a modified version of Duda's (1989) Purpose of Sport Questionnaire. Participants responded to items on both the TEOSQ and PPSQ using 5-point Likert-type agreement scales.

The TEOSQ had two factors, task-orientation and ego-orientation, which were both tested for validity and provided alpha coefficients of .86 and .83 respectively in the sample. Results from the PPSQ were run through a factor analysis which produced five subscales with factor loadings greater than .4: (a) fitness, (b) aggression, (c) status, (d) sportsmanship, and (e) financial remuneration. These five subscales accounted for $68.7 \%$ of the variance in overall purpose of soccer. Correlation analysis between the TEOSQ scores and PPSQ scored revealed two significant findings. Athletes who scored high on task-orientation and low on ego-orientation had scores correlating positively to high sportsmanship scores and negatively to high remuneration and aggression scores. Athletes who scored high on ego-orientation and low on task-orientation had scores correlating positively with high scores in financial remuneration and fitness.

The researchers also conducted a MANOVA using amateur or semiprofessional status as the independent variable and the five purpose of soccer factors as well as the 
task or ego orientation scores as the dependent variables. Significant differences were found in financial remuneration, status, and aggression, with semiprofessional players scoring higher, and sportspersonship and task orientation, with semiprofessional players scoring lower.

The results of the study echoed earlier findings that ego-oriented athletes view the purpose of sport more extrinsically and task-oriented athletes view it intrinsically (Duda, 1989; White, 1995). One surprise finding was the positive link between ego orientation and fitness. The study also lent credence to the argument professional athletes are more concerned with the financial and status rewards of playing sport than with the benefits of the sport itself. In addition, the results indicated the purpose of sport questionnaire does have validity for studies in other cultures. Prior to Carpenter and Yates (1997) work, the instrument had not been used outside the United States.

Treasure, Carpenter, and Power (2000) also examined the relationship between achievement orientations (task and ego) and perceived purpose of sport, this time using professional and amateur English rugby union players. As documented in earlier studies (Carpenter \& Yates, 1997; Duda, 1989; White, 1995) athletes conceptualize goals or achievement in sport in primarily two different ways, through task-orientation or egoorientation. These conceptions have been shown to affect athletes' perceived purpose of sport participation, with ego-oriented athletes displaying a stronger relationship with factors such as higher status, more aggression, higher self-esteem, and higher financial remuneration. Meanwhile, task-oriented athletes felt sport participation was more about mastering skills, living an active lifestyle, and sportsmanship. 
In their study, Treasure et al. (2000) collected data from male rugby players $(N=$ 179), 73 of whom played in the English first division and were paid to play, while 106 played in junior clubs and were not paid. All participants completed a 12-item Perception of Success Questionnaire and a 24-item Perceived Purposes of Rugby Questionnaire, modeled after the Purpose of Sport Questionnaire developed by Duda (1989). Responses from both instruments were recorded on 5-point Likert-type agreement scales. The Perceived Purpose of Rugby Questionnaire contained six sub-scales: (a)

sportspersonship, (b) status, (c) financial remuneration, (d) fitness, (e) aggression, and (f) peer relations. After a factor analysis was run on the data, however, the researchers eliminated one sub-scale, status, because of items loading on that factor also loaded significantly on at least one other factor. Two other items were also thrown out because of multiple factor loadings. The remaining 19 items loaded on to five factors and all produced satisfactory Cronbach’s alpha coefficients ranging from .64 to .88. The factors selected accounted for $66 \%$ of the variance in purpose of rugby.

Canonical correlation analysis revealed one significant function between achievement orientation and purpose of sport factors. Fitness, aggression, and financial remuneration were all positively related to those with high ego-orientation and moderately high task-orientation. The researchers ran a MANOVA with amateur or professional status as the independent variable, and achievement orientation and purpose of rugby factors as the dependent variables. They detected a significant effect. Separate ANOVAs revealed professional players scored significantly higher on the factors of task and ego orientation, plus the purpose of rugby factors aggression, financial remuneration, and fitness. Amateur rugby players scored significantly higher on sportspersonship. 
Based on their results, the authors suggested money is indeed a salient motivator of English rugby players, particularly those who are playing professionally. They also noted that as competition level increased (amateur to professional) aggressiveness became more important and sportspersonship less important. This finding highlights possible emphasis on a win-at-all-costs mentality as athletes progress up the athletic scale, from mass-participation sport to elite competition.

To summarize, athletes at all age and skill levels participate in sports for various reasons. Duda and Allison (1990) first suggested cultural and ethnic differences could account for difference perceived purposes for sport participation. Duda (1989) developed a scale to measure perceived purpose of sport participation, which offered seven different purposes. Several studies since the development of that scale have examined differences among groups in their view of sport and what it should do or accomplish for an individual. Results of these various studies confirm different groups view sport in unique ways. While no researchers have utilized the Purpose of Sport Questionnaire (Duda, 1989) to compare domestic and international student-athletes, to do so is justified based on this literature.

\section{Elite Sport Transitions}

As youth become more involved in sport, they move up a scale of attachment to the sport, from beginners to specializers to investors (Cote \& Hay, 2002) or from massparticipation sport to competitive sport to high performance sport (Green, 2005). This athletic progression or athlete transition brings with it qualitative social, physical, and mental adjustments for the athletes themselves. International student-athletes making the jump from their home nations to competition at the NCAA Division I level in the United 
States typically need to make such athlete transitions to have a successful experience.

Few researchers have looked at the athlete transition experience as young athletes move into a new realm of competition.

Chambliss (1989) conducted an ethnographic study of elite United States swimmers. Using qualitative means, he fulfilled a traditional participant-observer role, watching several elite level swimming competitions, including the Olympic team trials and the actual 1988 Olympic Games. Chambliss also recorded 120 interviews with elite swimmers and coaches. He began his investigation several years before the Olympic games, giving him a true longitudinal study and eliminating the bias of only examining the champion swimmers. He looked at all elite swimmers vying for a spot on the national team and was able to contrast their approach with his observed experiences of beginning and mid-level amateur swimmers. The researcher employed a literature review and discussion of findings with other experts to triangulate data.

From his research, Chambliss (1989) concluded little quantitative difference existed between elite swimmers and lower level swimmers. Instead, he posited the major difference is qualitative, meaning the training methods and approach taken by top swimmers was inherently different than the means taken by lower level swimmers. His conclusions included: (a) “excellence” is not the result of a purely unique personality which separates the elite swimmer from the average one, (b) simply increasing the quantitative training regimen by doing more work or moving the arms faster--but in the same motion, is not the key difference between mid-level and high level competitors, and (c) "talent" is not the major factor stratifying different levels of swimming. Chambliss argued the concept of "talent" really is a myth and elite swimmers do not possess any 
more of a natural gift for swimming compared to lower level participants. Instead, he illustrated key qualitative differences between levels of the sport including: (a) the physical swimming techniques taught and used, (b) the discipline required by elite athletes, and (c) the attitude of high-level competitors.

The investigator went on to state the stratification between levels of swimming is not continuous, but rather discrete. This means athletes desiring to jump to a new level must make a qualitative change in technique, discipline, and attitude to make such a leap. Higher level teams and coaching often include these distinct differences. The personalities and abilities of lower level swimmers are not better for competition than those of elite swimmers. Chambliss (1989) also argued talent does not lead directly to excellence for three distinct reasons: (a) other factors more accurately predict success, (b) talent or natural ability are labels placed on individuals after they have shown an ability to perform, and thus cannot be recognized until after the fact, and (c) numerous examples exist of elite athletic performance which overcame a distinct lack of "talent". Ultimately, Chambliss concluded elite performance is a result of: (a) mundane repetition of superior technique, (b) motivation through mundane goals for everyday performance (versus, for example, winning Olympic gold), and (c) making every endeavor, whether a practice session or an elite meet, mundane to eliminate nervous tension and provide ultimate comfort during performance.

Pearson and Petitpas (1990) examined the psychological transitions made by athletes, looking through the lens of developmental psychology and, in particular, the Schlossberg (1981) model of adult transition. Transition was defined as a change in assumptions about oneself and the world, resulting in a change in behavior or 
relationships, due to the occurrence of a particular event or nonevent. In the Schlossberg model, three factors influence transitions, their impacts, and outcomes: (a) the individual, (b) the individual's perception of the transition, and (c) the setting. The researchers reviewed the existing literature on the topic and identified key issues linked with psychological impact of athlete transition. They also outlined a preventative intervention program which could assist athletes forced into transitions.

Pearson and Petitpas (1990) cited three examples of athlete transition: (a) being cut from a team, (b) injury, and (c) retirement. Elite athletes who undergo one of the previously listed transitions often suffer from a lower ego involvement and loss of identity, which can develop into feelings of separation and loneliness, fear and anxiety, or loss of confidence. Athletes are particularly vulnerable to such transitional stress because their sense of industry and identity is highly focused on athletic performance. Human psychological development and maturation depends to some extent on exploratory behavior, but many elite athletes follow strict regimentation under close supervision, limiting their opportunities for such personal exploration. As a result, the researchers suggested elite athletes often do not "flesh out" their personal identities, which makes it more difficult for them to cope with particular transitions.

Pearson and Petitpas (1990) also outlined several barriers, as cited by other researchers, which limit successful transitions for elite athletes. The first barrier is the amount of time athletes have to prepare for the transition and the perceived evaluation of the transition. For example, injured athletes have little time to prepare for nonparticipation, which adds to the difficulty of the transition, but athletes moving from a lower level to a higher one have less difficulty with the transition because the move is 
seen as a positive transition. A second barrier is the physical environment and social context within which the transition takes place. How well a team or organization can service an athlete in transition or how well team mates and team personnel socially support the athlete in transition can dramatically affect the transition experience. Finally, personal characteristics of the athlete help or hinder the transition experience. Welleducated athletes seemed better equipped to deal with transition. Those athletes who exemplify what Pearson and Petitpas call "rugged individualism,” the concept of selfreliance and disdain towards requesting outsiders' assistance, are limited in their ability to deal with difficult transition. Since many athletes embrace the ideal of "rugged individualism” in their training and competition, athletes may be more likely to reject assistance or may feel inflated senses of entitlement.

Using the Schlossberg (1981) model, Pearson and Petitpas (1990) cited six characteristics making the transition experience difficult for athletes: (a) identity based exclusively on athletic performance, (b) large gaps between level of athletic aspiration and athletic ability, (c) little prior experience with transition, (d) emotional and/or behavioral deficits, (e) ability to form and maintain supportive relationships, and (f) lack of coping resources, both physical and emotional. The researchers suggest the use of preventative programs, such as the United States Olympic Committee’s Career Assessment Program for Athletes, to help elite athletes in their transition processes.

Riemer, Beal, and Schroeder (2000) examined the social processes affecting elite female college athletes and whether these social processes impacted their commitment to academics. Past studies, most notably Adler and Adler (1985), suggested the social conditions established by commercialized sport at the NCAA Division I level affected 
student-athletes in several ways, such as isolating them from the rest of the student body and emphasizing athletic achievement over academic achievement. Meyer (1990) countered that while elite female athletes socialized primarily with teammates and other athletes on campus, they placed more emphasis on academic achievement, primarily because they gained far less recognition from their athletic achievements.

Riemer, Beal, and Schroeder (2000) tested this assertion, examining how the level of athletic commercialization and gender of the student-athletes impacted the college experience, especially academic adjustment. The researchers used qualitative means, interviewing several female student-athletes at two different NCAA Division I schools. One school was a small, private, west coast institution, while the other was a large, public, midwest university. The researchers selected two sports, tennis and basketball, because the two represented both team and individual sports, and sports in which women could continue their athletic careers professionally. The researchers asked team members to volunteer for a single, anonymous interview. At the west coast school, all seven members of the tennis team and 13 of 14 members of the basketball team participated. At the midwest school, four of six tennis players participated, as did six of nine basketball players. Each student-athlete was interviewed privately, with the conversation recorded, and asked a series of questions regarding: (a) demographics, (b) athletic experiences, (c) academic experiences, and (d) social experiences. After the interviews, the researchers analyzed the transcripts separately and developed a list of emerging themes. These themes were then compared. The researchers pulled out common themes, than reanalyzed the transcripts to determine further emerging themes upon which they could agree. 
Three major themes developed from the data: (a) view of career termination for tennis players, (b) professionalization of college sport for basketball players, and (c) social isolation for all female student-athletes. Over half of the tennis players interviewed were international students, most of whom had ambitions of turning professional but were unsuccessful in this endeavor, electing to play collegiate tennis in the United States. Several of these players indicated tennis had been their motivation in life, but were now looking at other professional career choices after their experiences in college. Several of the domestic tennis players indicated college tennis was likely going to be the peak of their career. Many of the tennis players saw their education leading to a start in a profession other than athletics. The basketball players, on the other hand, saw their scholarships as an obligation or exchange in which they would dutifully perform the actions expected of an athlete so their college tuition would be paid. They saw the experience much like a job, although several indicated they hoped to either play professionally after school or become involved in coaching. Athletes from both sports indicated they felt socially isolated from the rest of the student body on campus, particularly the international student-athletes, who were also separated by cultural and language barriers. The researchers also found the female athletes in their study prioritized academics alongside their athletic participation, counter to Adler and Adler’s (1985) findings with elite male college athletes, but in agreement with Meyer (1990).

Ryska (2001) examined how acculturation of Mexican-American adolescents affected their motivation of playing sports in middle school and high school, highlighting differences in gender. Past studies investigated how goal motivation, which Nicholls (1989) breaks down into task-related motivation and ego-related motivation, impacted 
achievement, skill, perceived confidence, and sportspersonship. In this study, Ryska examined goal motivation within-groups (gender) of a particular ethnic population (Mexican-Americans).

The study included 163 participants split nearly in half by gender (male $n=83$, female $n=80$ ). Ryska (2001) used a three-part questionnaire extracting information about: (a) motivational orientation toward sports, (b) ethnic acculturation into mainstream society, and (c) demographic information. To gather motivational information, he used the Task and Ego Orientation in Sport Questionnaire (TEOSQ) (Duda \& Nichols, 1989). The TEOSQ scale was comprised of 13 questions measuring task-related and ego-related responses, which were assessed through 5-point Likert-type agreement scales anchored by 1 (strongly disagree) to 5 (strongly agree). The scales were tested for reliability and resulted in acceptable scores for both task-related $(r=.86)$ and ego-related $(r=.89)$. To collect acculturation data, the Acculturation Scale for Hispanics (ASH; Marin, Sabogal, Mari, Otero-Sabogal, \& Perez-Stabel, 1987) was used. The ASH scale consists of 12 items, representing three dimensions of acculturation: (a) language, (b) media, and (c) ethnic social relations. ASH responses were scored on a 5-point Likert-type scale anchored by 1 (only Spanish) to 5 (only English) or 1 (all Latinos/ Hispanics) to 5 (all Whites/Anglos). Total reliability for the combination of all three dimensions was satisfactory $(r=.89)$, as were each section individually of language $(r=.82)$, media $(r=$ .74), and ethnic social relations $(r=.73)$. Demographic data collected included gender, age, sports played, number of years played, and academic grade.

A confirmatory factor analysis validated the factor structure of the TEOSQ scores. Tests were also run for multicollinearity among the control variables. A series of separate 
simultaneous multiple regression analyses were then performed. One analysis regressed motivational orientation on acculturation level and gender. Media use $(\beta=.265, \mathrm{p}<.001)$ and ethnic social relations $(\beta=.188, \mathrm{p}<.001)$ were significant predictors or task-related motivation for males, while language $(\beta=.336, \mathrm{p}<.01)$ and ethnic social relations $(\beta=$ $.259, \mathrm{p}<.05$ ) were significant predictors of ego-related motivation for females.

The researcher offered several conclusions regarding the gender difference detected in the study. The literature demonstrates the more youth become acculturated, the less their sports motivation is ego-related. Since males scored significantly higher in the acculturation scores, it makes sense that their task-related motivation was higher, while females had higher ego-related motivation, which follows their lower acculturation scores. Ryska (2001) also suggests some difference may lie in a difference in how sport participation is perceived by Mexican-American culture as a whole.

In summary, as athletes progress throughout their careers, they move through levels of athletic competition, from low-level, mass-participation sport all the way up to elite sport. As these athletes make jumps to different levels, they must make transitions, not only physically, but psychologically. Some researchers have examined the effect of these athlete transitions, noting the extent of the transition and pre-transition preparation can affect successful athlete adjustment. A cursory understanding of the phenomenon of athlete transition is necessary to examine college student-athletes, particularly those coming from other nations, because such high-level athletes would certainly go through multiple levels of athlete transition. 


\section{Student-Athlete Adjustment to College}

A common athlete adjustment period for elite United States athletes is the jump from high school athletics to college. This transition can affect athletes in many ways socially, academically, and athletically. Several studies led primarily by the researchers Pascarella and Terenzini (1991, 1996, 1999) examined different aspects of adjustment for college students, including a series of studies focusing on how athletic participation affects academic achievement in college. While these studies have looked at this issue empirically, often using grade point average and academic test scores as dependent variables, several other researchers have sought to discover the social and psychological impact of sport participation on college students. The team of Adler and Adler conducted some of the most extensive studies, including a four-year ethnography, which resulted not only in an article summarized below (1985), but also a book entitled Backboards and blackboards: College athletes and role engulfment (1991). Several other investigators have followed in their footsteps.

Howard-Hamilton and Sina (2001) summarized the existing literature regarding theories of student development in college and how participation in athletics affects such development. The researchers began with a look at psychosocial theory of development, as espoused by Erikson (1980) and others, which addressed how individuals develop definitions of who they are and what kind of relationships they have with others. Howard-Hamilton and Sina focused on the stages of industry and identity, which are the most relevant stages of development for traditional age college students. They suggested most athletes develop positive senses of industry, or productivity, and ego identity because of their athletic prowess and accomplishments. Unlike many other college 
students, however, these positive self-concepts are dependent on their on-court or onfield success, which the researchers point out, can lead to a limited scope of what defines success and achievement in all aspects of life. The intense focus on athletic accomplishment overshadows academic and social development. The researchers also stated the heavy reliance on coaches and other athletics department personnel to lead the student-athletes through the college process limits their development of independence and autonomy.

The cognitive development of individuals focuses on how people think and how meaning is made from experience. The researchers argued student-athletes are often told by coaches what right or appropriate behavior is, enforcing a belief that all situations have correct or incorrect responses. They contend this relationship limits the studentathletes' ability to develop a personal cognitive understanding of the ambiguities people encounter on an everyday basis. They also argued this reliance on authority figures limits moral development of the student-athletes.

Howard-Hamilton and Sina (2001) also gave an overview of the impact athletic participation had on student-athletes' cognitive growth and development. Studies by Astin (1993) and Pascarella, Edison, Nora, Hagedorn, and Terenzini (1996), and Pascarella, Truckenmiller, Nora, Terenzini, Edison, and Hagedorn (1999) have shown participation in the male sports of basketball and football have negatively impacted cognitive development, but such a relationship did not exist for females athletic participation, nor for male participation in non-revenue sports. Additional research has shown student-athlete cognitive development can be both positively and negatively influenced by the way athletes are perceived by professors and other students on campus. 
Howard-Hamilton and Sina (2001) called for more cooperative work between student affairs administrators and athletics departments to improve psychosocial and cognitive development of student-athletes. They also suggested student-athletes be encouraged to participate in other campus activities and be given more ability to make personal choices, which would enhance their advanced psychological and social development. Such options would also improve the holistic health and well-being of the student-athletes.

Pascarella, Truckenmiller, Nora, Terenzini, Edison, and Hagadorn (1999) examined the cognitive impact of participation in intercollegiate athletics. Prior research indicated mixed results regarding the relationship between intercollegiate athletic participation and perception of college experience. Researchers in previous studies connecting athletic participation and academic achievement used grade point average as an independent variable. Pascarella et al. pointed out many pre-college factors, as well as university and field of study differences, which could impact grade point average but were not accounted for in past research with college student-athletes. The goals of their study included estimating the cognitive impacts of college sport participation after two and three years of college attendance and control for academic experience and background characteristics of student-athletes.

In their study, Pascarella et al. (1999) selected a sample from 18 different fouryear universities. A stratified random sampling procedure was employed to ensure participants came from public and private schools, different geographic locations, commuter and residential schools, urban and rural schools, and all three levels of NCAA competition. The researchers administered four-dimension academic tests called the 
Collegiate Assessment of Academic Proficiency (CAAP) to 3,331 first year college students in the fall of 1993. Follow-up data was collected at the end of the school year, with 867 men and 1,549 women completing the second set of tests, plus the College Student Experiences Questionnaire (CSEQ), which is used to evaluate college experience. Two dimensions of the CAAP tests were then administered a third time during the students second year of college where 562 men and 1056 women from the original sample completed the follow-up tests. The other two dimensions of the tests were administered during the third year of the study and 390 men and 747 women who completed the second-year tests completed the instrument a final time. Students were given a stipend, ranging from $\$ 35$ to $\$ 50$ for each time they completed the tests. During the second and third years of the study, approximately $25 \%$ of the male respondents were athletes, while approximately $14 \%$ of the female respondents were athletes.

The researchers employed linear regression analysis, with test scores as the dependent variables and athletic participation as the independent variables. They also ran a second set of statistical tests to develop interaction terms between athletic participation and several pre-college conditions, as well as school student body cognitive level and division of NCAA participation. Results of the analyses demonstrated no significant difference between CAAP test scores for the student body and males from non-revenue sports, but did reveal significantly lower scores in all four dimensions for male athletes in basketball and football, compared to all other male athletes. Only one of the four CAAP tests, reading comprehension, resulted in any significant difference between female athletes and their non-athlete counterparts. 
The authors found no differences in the results when controlling for pre-college conditions, which led them to suggest something within the sports of football and basketball triggered lower cognitive scores. They also noted the lower scores were true at all three levels of NCAA competition for football and basketball. Pascarella et al. (1999) suggested perhaps those two sports required a larger "psychic" investment of time and energy, which limited the athletes' dedication to academic issues as compared to other sports, but further investigation in the matter was necessary.

Adler and Adler (1985) examined the connection between elite college athletes playing at the NCAA Division I level and their corresponding academic performance. Previous studies had spelled out conflicting claims, some demonstrating college athletes had higher graduation rates, higher GPAs, and lower attrition rates than non-athletes, while other studies revealed the direct opposite. Adler and Adler sought to uncover the real relationship between "big-time" college athletes and university academics by conducting a four-year ethnographic study with a single high-profile NCAA Division I men's basketball team.

In their study, Adler and Adler (1985) utilized the technique of participantobservation, gathering data through interviews with players and coaches as well as field observations. Peter Adler became the team sociologist, gaining in-depth access to the players and coaches, while Patrica Adler remained on the outside, helping to analyze and detect patterns emerging from the data collection. Their data collection site was a private, mid-south-central university with approximately 6,000 students. The school had a nationally-ranked men's basketball program, but was not considered one of the traditionally elite powerhouses of college basketball. 
The researchers uncovered several interesting facts about the program. Many athletes came into the program with high academic expectations, including full intent to graduate, despite often having poor scholastic preparation in high school. This belief stemmed from recruiting pitches from coaches who emphasized obtaining a diploma and from the fact that the student-athletes graduated from high school. They often believed if they simply "put the time in” at college, they would gain the same result. The researchers found early on, most participants thought they would have satisfactory performance in school and would ultimately earn their degree in their chosen major, which for nearly half the participants in the study began in a pre-professional program. After their freshman year, however, many participants found themselves doing poorly in school for several reasons. One reason was the large time commitment to their sport, which often allowed them far fewer hours for academic work, or when they did have time, they often complained of physical fatigue which made studying difficult. In addition, Adler and Adler (1985) found many of the participants uninvolved in academic decision-making processes because assistant coaches or other administrative personnel made all academic decisions, such as selection of courses, selection of major, registration for classes, and checking with professors on academic progress. Because athletes were not involved in these decisions, many adopted a behavior of non-engagement with most aspects of their academics. Many expected to be walked through their entire academic process, and therefore did not always take responsibility for properly completing course work, thinking exceptions would be made for them. They also received far more reinforcement for their athletic performance than their academic one. 
In addition, the researchers found many of the athletes suffered social isolation on campus. Most of the athletes lived in athletics housing, ate meals separately, studied in athletics-sponsored sessions with athletics tutors, and spent much of their practice time and free time with other athletes. This limiting social experience meant norms, particularly academic norms, were established by other athletes or athletics personnel. The result, as stated by Adler and Adler, “made their athletic reality dominant and distanced them from any academic inclinations they may have had” (1985, p. 246). Athletes felt professors either were very supportive or held a disdain for athletes, meaning most participants only took certain courses from particular instructors, which also reinforced their social isolation because the classroom was filled with other athletes. Several athletes were also encouraged to take physical education or recreation courses which were not challenging or did not prepare them for more difficult academic courses later in their career.

This perception of academic progress and social isolation resulted in many athletes shifting blame away from themselves for academic failure. It also meant many athletes changed majors, particularly out of pre-professional degrees and into less academically demanding ones. As a result, many athletes simply took just enough academic work to stay eligible and became much less motivated by degree obtainment. The authors stated their research showed a widening gap between athletes' academic ability and schools' academic expectations. They suggested three major changes to the college athletics landscape: (a) a reduction in the celebrity status of athletes; (b) removal of athlete-only dorms, meals, and study tables; and (c) provision of more academic role models for athletes. 
Killeya-Jones (2005) examined psychological role conflict for elite college student-athletes. She argued student-athletes identify themselves as both athletes and students, roles that can fight for the same "temporal and psychological resources" in a college setting. How individuals manage this conflict can have an impact on their ability to adjust and be successful in college. Neither of the two roles can be rejected, but rather must be reconciled in some way for the student-athlete to experience success.

In order to define identity roles among student-athletes, the researcher used the Hierarchical Classes model (HICLAS; DeBoeck \& Rosenberg, 1988; Rosenberg \& Gara, 1985) which places individuals and groups into a series of matrices based on selfperceived psychological elements such as role occupancy, role structure and organization, and role meaning. The sample for this study included 40 male football players from a single NCAA Division I university. Of the 40, 20 were upperclassmen, representing a response rate of $44 \%$ of eligible members of team. The other 20 participants were incoming freshman, representing $81 \%$ of the incoming class who had not started school at the time of the study. Participants filled out a survey containing measures of: (a) demographics, (b) anxiety, (c) depression, (d) self-esteem, (e) satisfaction, and (f) identity collection. The anxiety scale produced a Cronbach’s alpha rating of .89, the depression scale .91, and the self-esteem scale .77. All three scales were previously developed and tested. The satisfaction scale consisted of three single-item questions asking how satisfied student-athletes were with academic performance, athletic performance, and life in general.

Results of a correlation analysis confirmed the hypothesis that the greater the discrepancy between perceived roles as a student and as an athlete, the more negative the 
psychological adjustment score. A lower discrepancy score correlated significantly in a positive direction with self-esteem $(r=.40, p<.05)$, life satisfaction $(r=.39, p<.05)$, and academic satisfaction $(r=.45, p<.01)$, while correlating in a negative direction for anxiety $(r=-.41, p<.05)$ and depression $(r=-.46, p<.05)$. In addition, lower discrepancy scores between the two roles significantly correlated with positive elaboration of the student role $(r=.61, p<.01)$.

This study strengthened the assertion that the more student-athletes can integrate their dual roles, the more positive their adjustment to college will be and the more satisfied they will be with their experience. Higher scores in just one of the two roles did not correlate with high life satisfaction scores. The study had implications for college coaches and administrators who should strive to develop improved commitment to student roles. The athlete role is typically positively reinforced at the NCAA Division I level, while the student role is not emphasized. Killeya-Jones (2005) suggested coaches and administrators promote the student role, not at the expense of diminishing the athletic role, but in separate ways such as offering academic help outside of the athletics department, promoting the student role in a positive manner, allowing adequate time for school work, and discouraging large cohorts of athletes to take the same classes. The researcher recommended future studies in this area focusing on large groups of studentathletes at multiple schools, and also examining between group differences based on gender and sport played.

Kimball and Freysinger (2002) examined the relationship between stress, college student-athletes, and leisure activity, which in the case of this study was intercollegiate athletics. Typically, leisure activity in the form of physical recreation has been viewed in 
the literature as a buffering or mediating agent to stress. Some recent studies, however, suggest leisure can be a stressor in itself. In looking at the demands of intercollegiate NCAA Division I sport participation, the authors tested whether sport added to stress levels or decreased them.

To conduct their study, Kimball and Freysinger (2002) utilized qualitative methodology, conducting semi-structured interviews with 14 student-athletes at a single NCAA Division I institution. The sample selection was both convenient and purposive. Half the sample was male. Seven participants classified themselves as Caucasian; while five considered themselves African-American and two said they were bi-racial. Participants represented all academic class levels and nine different sports. The lead researcher interviewed each participant individually, recording the interview and taking observation notes. Participants were asked about their daily routines and about their experiences with stress. After the interviews, data was transcribed, coded and analyzed for themes. The researcher met with each participant a second time to review the transcription and talk about or clarify additional information. The second author in this study also reviewed all final transcripts to provide additional analysis. The researchers then discussed their thematic findings.

Similar to other studies, Kimball and Freysinger’s (2002) research revealed athletes displayed characteristics of a self-determination and "hardy" personality. Such attributes often led the participants to tackle potential stressful situations as a challenge, similar to how they approach sport pressure in the athletic arena. The student-athletes often stated they dealt with academic, athletic, and social stresses through selfdetermination, self-control, and self-confidence. When situations, particularly their sport 
participation, felt beyond their control, however, the student-athletes saw collegiate sport as a stress inducing activity. The more specific aspects of sport participation developing into stressors included: (a) coach and teammate relationships, (b) social or racial inequality, and (c) gender stereotyping. Student-athletes also saw their sport as stressful when they felt a lack of competency in their ability, such as learning new playing techniques or performing poorly in a contest.

Kimball and Freysinger (2002) also found evidence of sport as both a stress buffer and inducer in matters of social support. Participants commented that teammates provided friendships and motivational competition helping to overcome daily stresses. Sport was also found to be the cause of certain social stressors, such as race and gender tensions. African-American student-athletes felt isolated in both their sport and on the predominately white college campus, women felt pressure to conform to unhealthy body images, and several students felt stereotyped as athletes not interested in doing well in school.

Such data confirmed findings in the literature that stress in leisure activity was both dynamic and transactional. The transactional process means stress does not stem directly from the event or the individual but depends on both. The authors cited two methods of coping with stress, emotion-focused coping (EFC) and problem-focused coping (PFC). They suggested the student-athletes in their study could see sport as both a stressor and stress-reducer, but viewed it from a personal perspective, not an environmental one. Student-athletes make individual adjustments, not situational adjustments, to deal with the stress of sport. Environmental or social factors such as race, gender, or social class, played a large role in the athlete’s perception of daily stress, but 
the student-athletes often failed to recognize the interaction or connection between these factors and the stress in their lives.

To summarize, several researchers have examined the impact of participation in college athletics on the mental and social adjustments of student-athletes. Adler and Adler (1985) were the first to find that adjustment for college student-athletes at the elite level of competition was often quite different than that experienced by college students who did not participate in athletics. Several other investigators echoed Adler and Adler's findings that student-athletes often have academic and social decisions made for them by athletics department personnel. Because of this, and the high emphasis placed on athletic achievement, student-athletes in high-profile settings off struggled with academic and social identity roles.

\section{International Student-Athlete Adjustment to College}

In regards to adjustment, international student-athletes fall under a unique set of circumstances. As athletes, they must deal with the athletic transitions outlined above. As cross-cultural visitors to another country, they must also adjust socially and culturally to living in a new environment. They face the demands of studying at a new university, often using a language that is not their native tongue. A handful of studies have been conducted regarding international student-athlete adjustment. Ridinger (1998) examined student adjustment to college, using domestic and international student-athletes as two of her primary sample populations. Ridinger and Pastore (2000a; 2000b) looked at adjustment factors for international student-athletes and developed a framework for further testing these adjustment factors. Before their work, Bale $(1987 ; 1991)$ and Stidwill (1984) were the only two individuals to look at the phenomena of international 
student-athletes in any sort of scientific fashion. Craven (1994) did not look specifically at international student-athletes, but did investigate factors of effectiveness on athletes and coaches who traveled cross-culturally.

These studies depicted international student-athletes as often being better adjusted than their non-athlete international compatriots and often times more academically prepared than their fellow domestic teammates. The studies also begin to narrow the field of the most significant adjustment factors for international student-athletes, but certainly left room for further study. A review of these studies on international student-athletes follows.

Ridinger (1998) examined adjustment to college levels for several subgroups, including: (a) domestic student-athletes, (b) international student-athletes, (c) domestic students, and (d) international students. To aid with her research, Ridinger developed a conceptual framework, which she called the student-athlete acculturation model, based on a review of the literature. In her model, Ridinger used the five adjustment levels outlined by Baker and Siryk (1984), which include: (a) academic adjustment, (b) social adjustment, (c) personal-emotional adjustment, (d) institutional attachment, and (e) fullscale adjustment.

Ridinger (1998) administered the Student Adaptation to College Questionnaire (SACQ; Baker \& Siryk, 1989) and an additional instrument which she developed called the Factors Associated with Student-Athlete Adjustment to College (FASAAC), to students at a large Midwestern University $(N=1,015)$. The SACQ had 67 items and the FASAAC had 48 items. Participants rated items on 9-point, Likert-type agreement scales on the SACQ and 5-point, Likert-type agreement scales on the FASAAC. Among the 
sample population, 379 participants were domestic students and 306 responded for a rate of $81 \%$, while 317 were international students and 81 responded, for a rate of $26 \%$. Among student-athletes in the sample, 269 were domestic student-athletes and 86 responded, for a response rate of 32\%, while 50 were international student-athletes and 18 responded, for a rate of $36 \%$.

The researcher used MANOVAs to compare group means of the five adjustment levels based on population subgroup and found international students were the least welladjusted, while in several respects, international student-athletes were the most welladjusted to college. Of particular interest was the finding international student-athletes were significantly better adjusted academically than domestic student-athletes. Ridinger (1998) also utilized a series of MANOVAs to look at adjustment scores for different demographic variables and found female student-athletes were more well-adjusted to college than male college athletes and that Asian international students were the least well-adjusted group. The use of the FSAAC allowed Ridinger to detect relationships between antecedent and outcome variables on college adjustment level. Through the use of several MANOVAs, she found out-of-state student-athletes had the most realistic expectations of college experience and international student-athletes felt a greater cultural distance in their college experience than did in-state or out-of-state student-athletes.

Ridinger and Pastore (2000a) examined how well international student-athletes adjusted to attending United States universities compared to international non-studentathletes, domestic student-athletes, and domestic non-student-athletes. Overall, they received 443 usable survey responses from students from a large, Midwestern university. The researchers did not report total response rates, but admitted their target group of 
international student-athletes was quite low (16 responses from a population of 50). The survey used in the assessment was the Student Adaptation to College Questionnaire (SACQ), a 67-item self-report questionnaire designed to assess how well students adapt to the demands of the college experience.

The independent variables examined in the study included gender, ethnicity - six levels: (a) Asian, (b) African-American, (c) Caucasian, (d) Hispanic, (e) Native America, (f) other, and student sub-group - six levels: (a) in-state non-athlete, (b) in-state athlete, (c) out-of-state non-athlete, (d) out-of-state non-athlete, (e) international non-athlete, and (f) international athlete. The dependent variables used to assess student adjustment were classified into five headings: (a) full adjustment, (b) academic adjustment, (c) social adjustment, (d) personal adjustment, and (e) attachment to the university. Ridinger and Pastore (2000a) then conducted three ANOVAs, first with student sub-group and student adjustment ( 6 X 5), then with gender and student adjustment ( 2 X 5), and finally with ethnicity and student adjustment (6 X 5). Significant findings included international student-athletes having the highest mean scores for overall adjustment to college compared to the other five student sub-groups, and the highest mean score for the academic adjustment sub-scale, which was particularly interesting since international non-athletes had the lowest academic adjustment scores. In fact, the researchers found statistically significant differences for full scale and the four other sub-scale headings based on the sub-group categories. The researchers concluded international studentathletes did not have a difficult time adjusting to college compared to other types of students, but again reiterated their small sample size in international student-athletes. 
Ridinger and Pastore (2000b) also presented a conceptual framework to identify factors associated with international student-athlete adjustment to college. In 1996, the National Collegiate Athletic Association (NCAA) released data showing a total of 8,851 international student-athletes competing on rosters of all three NCAA divisions (1996). The investigators stated that despite the large number of international student-athletes competing in the United States, very little research had been conducted to assess how these student-athletes adjusted to attending United States colleges and universities. Such knowledge is important for athletic administrators and coaches, as well as the studentathletes themselves. Ridinger and Pastore suggested positive adjustment to college can translate to other positive attributes such as better academic performance and comfort level, which is beneficial to all parties.

Adjustment to college has been linked to academic performance (Baker \& Siryk, 1984b, 1989), while athletic participation has been linked to increased persistence of staying in school, satisfaction with college, and academic achievement (Astin, 1984; Pascarella \& Smart, 1991; Ryan, 1989), although no research has been conducted linking adjustment to college and athletic performance. The researchers also examined business literature, which demonstrated a positive link between adjustment and performance, including how fast the adjustment occurred, for managers working in new environments overseas.

Ridinger and Pastore (2000b) used three tenants to develop their framework (see Figure 1) namely: (a) antecedents of adjustment, (b) adjustment to college, and (c) outcomes that may be associated with the antecedents and/or adjustment to college. Additional variables which must be accounted for included demographics such as country 
of origin, gender, athletic division of NCAA, and sport played. After examining studies by Bale (1991), Craven (1994), and Mendenhall and Oddou (1985), among others, Ridinger and Pastore developed a model of antecedents of adjustment for international student-athletes with four dimensions: (a) personal, (b) interpersonal, (c) perceptual, and (d) cultural distance. The personal dimension deals with the athlete's self-efficacy (self assuredness), both athletically and academically. The interpersonal dimension pertained to attributes which allow the athlete to communicate and interact with other teammates, classmates, coaches, and administrators. The perceptual dimension referred to how an athlete saw the school and/or the athletic environment encompassed there. The culture distance factor encompassed how different the campus culture is compared to the international student-athlete’s home environment. 
Figure 1

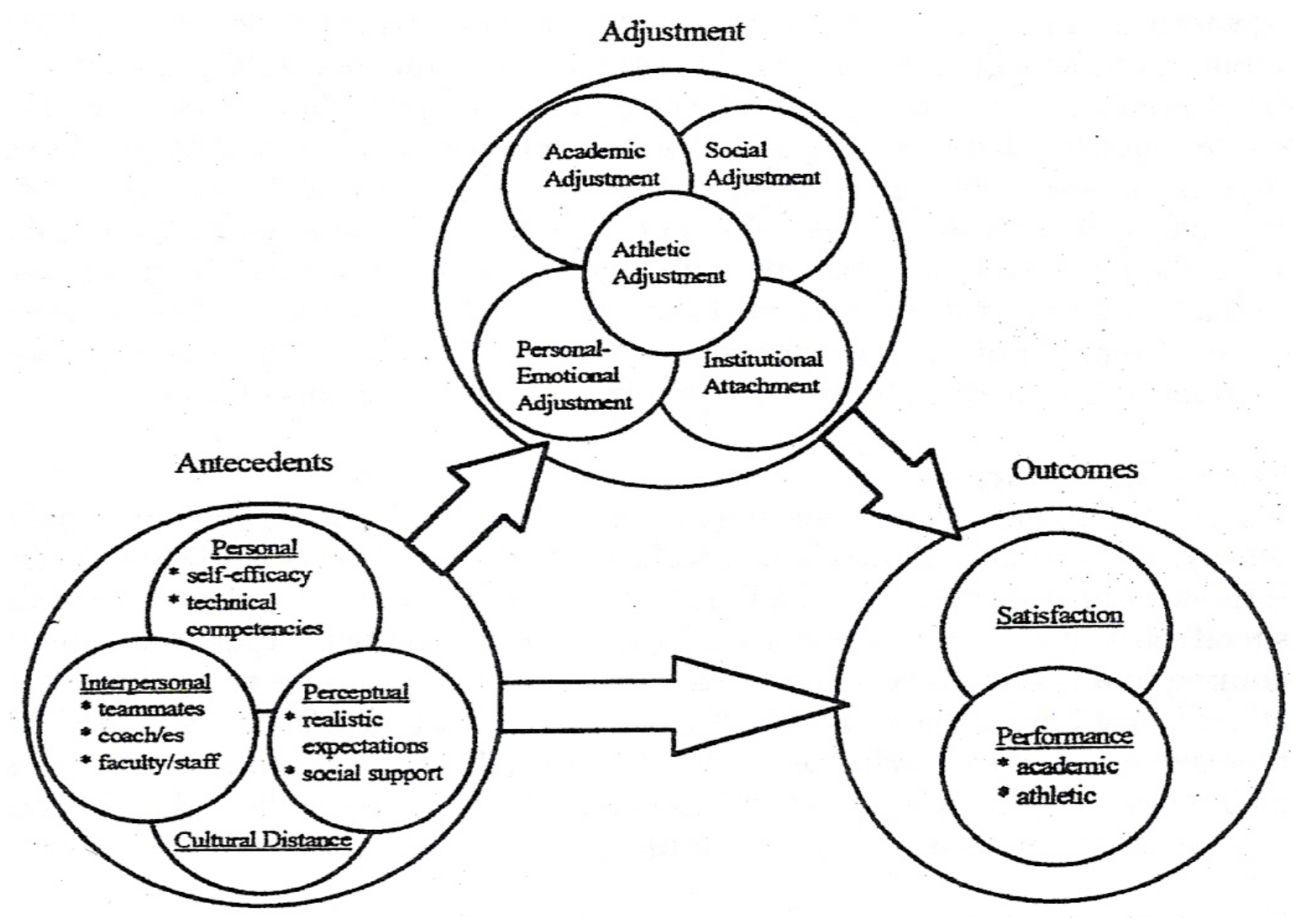

Figure 1. Ridinger and Pastore (2000b) created a model of adjustment to college for

international student-athletes depicting four antecedent factors, five types of adjustment, and two outcome levels to define successful adjustment to college. 
Ridinger and Pastore (2000b) stated adjustment to college has been studied both quantitatively and qualitatively, but never with international student-athletes used as a variable. They argue the Student Adaptation to College Questionnaire (SACQ) developed by Baker and Siryk (1989) is the best quantitative tool to measure student adjustment to college, but it does not incorporate athletic participation. In their model, they list five adjustments necessary for international student-athletes: (a) academic, (b) social, (c) athletic, (d) personal-emotional, and (e) institutional. Regarding outcomes, the investigators listed three of importance for international student-athletes: (a) academic performance, (b) athletic performance, and (c) overall satisfaction with college. Ridinger and Pastore (2001) conducted a study to gauge perceptions of collegiate coaches regarding the recruitment of international student-athletes. College athletics coaches and administrators possess varying viewpoints when it comes to landing international student-athletes to play at their school. Some argue against recruiting such athletes because it is expensive, it gives teams that can afford to do so an unfair advantage, takes spots away for United States born athletes, and the process can train foreign born players to the point that they go on to win international competitions, defeating United States' athletes. Critics also argue foreign-born athletes can be a distraction to other United States-born team members because they garner more attention and are not familiar with United States cultural customs, and they can be seen as only attending college for athletics, not for academics. Proponents of recruiting international student-athletes contend foreign-born athletes are often more prepared academically and athletically and they promote diversity and cultural awareness. Coaches who recruit international student-athletes do so because such athletes are often strong performers who 
make their teams more competitive and because international student-athletes perform well in the classroom and are often grateful for their scholarship opportunities.

The researchers distributed 368 surveys to coaches at National Collegiate Athletic Association (NCAA) institutions at all three divisions (I, II, and III), and at National Junior College Athletic Association (NJCAA) schools. Stratified random sampling was used to select the coaches. From the survey distribution, 146 participants returned the questionnaires, for a response rate of $39.6 \%$. The survey contained 31 items with responses marked on a 7-point, Likert-type scale anchored by "strongly agree” to "strongly disagree.” A panel of 14 experts analyzed the questions and 132 coaches pilot tested the survey, providing validity to the instrument. Through factor analysis, the investigators reduced the items to four factors: (a) recruiting, (b) international studentathletes, (c) attitude, and (d) adjustment issues. These four items became the dependant variables. The independent variables included six demographic variables: (a) athletics association (NCAA or NJCAA), (b) division (I, II, or III), (c) institution type (public or private), (d) gender (male or female), (e) coach of men's/women's/both teams (men's teams, women's teams, or both men's and women's teams), and (f) sport (basketball, field hockey, ice hockey, soccer, swimming, tennis, and track). Twenty-one items had factor loadings of .30 or higher. Three of the four factors each possessed reliability coefficients of .75 or higher, while the fourth, "adjustment issues", had a coefficient of .56 .

Six MANOVAs were run on the six independent variables. Ridinger and Pastore (2001) performed Scheffe's post hoc analysis on any significant findings. Noteworthy results included: (a) Division I coaches rated the attitude factor higher than Division II 
coaches, (b) public institutions rated the international student-athletes factor higher than private institutions, and (c) track coaches rated the recruiting factor higher than basketball or soccer coaches. The researchers suggested further study to investigate why these differences existed.

Bale (1987) examined the ways international student-athletes make decisions about migrating and attending United States universities and how they react to their sojourn in a new environment, including their adjustment to different athletic, academic, and social situations. The researcher mailed 200 questionnaires to randomly selected European athletes who were either currently or previous on an athletic scholarship at United States universities. Of those, $46.5 \%$ responded ( $n=93), 70 \%$ of which were male. Respondents lived in England, Sweden, Belgium, and the Netherlands. The questionnaire included both questions requiring ranked responses and open-ended responses.

Bale (1987) used descriptive statistics from the sample for his analysis. He also used a model outlining a locational decision making process, developed by Brown and Moore (1970) and Roseman (1971). Key findings included: (a) how European studentathletes were recruited, (b) what nationality were their friends, (c) what was their satisfaction level with migration, in terms of academics, athletics, and social life, (d) their levels of loneliness and homesickness, and (e) their overall experience. Of all respondents, $51.6 \%$ came to the United States because of a contact made by a university representative, while another $39.7 \%$ were contacted by friends who had been at the school. Also, 73.9\% of respondents received more than one scholarship offer. When international student-athletes chose their school, Bale found warm weather, having other international student-athletes, and the amount of the scholarship were the most important 
factors in determining which school they would attend. Nearly one-third of respondents also noted the amount of time the coach spent recruiting them played a role in their decision. Results also demonstrated 39.5\% of British respondents singled out an American student-athlete as their best friend at school, while 32.1\% listed another British student-athlete.

Of the 93 respondents to the survey, 57 had attended school in the United States, but were no longer there. The investigator found $42 \%$ of these students left after just one or two years attendance. Participants noted homesickness and disagreement with coaching styles as the main reasons for not continuing. Most respondents (58\%), though, did stay longer than three years, while $60 \%$ had either obtained or expected to obtain a degree, although Bale (1987) noted a few respondents mentioned differences in qualifications between United States university degrees and those from Europe, particularly England. Degrees from the United States were often not viewed as highly as a comparable degree in Europe. The research also demonstrated student-athletes felt coaching, athletic facilities, and their own improvement all existed at satisfactory or better levels at their United States colleges for the majority of respondents. In a social adjustment context, Bale did point out 13\% of international student-athletes personally experienced discrimination, while nearly one-fifth (19.3\%) found more discrimination in the United States than expected. The majority of respondents felt homesick or lonely at times, but less than $6 \%$ said it occurred "very often”. Thirty-two percent cited financial problems, while 31.2\% mentioned dealing with the coach, 26.9\% personal depression, $24.7 \%$ adjusting to climate, $20.4 \%$ dealing with college administration, and $20.4 \%$ lack of motivation for study when listing the major problems they battled in an open-ended 
question on the survey (respondents could list more than one issue). Finally, the researcher asked respondents to rate their overall experience of their sojourn to the United States on a scale of 1 to 100 , with 100 being a wonderful experience. He found a mean score for all respondents of 87.

Craven (1994) examined the most important factors in determining a successful cross-cultural experience for people involved in international sport experiences. Craven suggested several personal attributes which ease cross-cultural adjustment for international athletes and coaches: (a) respect of host nationals, (b) non-judgmental perspective, (c) flexibility, and (d) people-orientation. Most individuals who travel internationally for sport display high competencies in their field, but Craven argued athletic ability is just one of many issues determining a successful transition. She categorized determinants of cross-cultural effectiveness into four groups and ranked them in priority order as follows: (a) interpersonal skills, (b) cross-cultural interaction/adjustment, (c) professional competency, and (d) personal qualities. The author suggested those who recruit and sponsor foreign coaches, athletes, and administrators look for people who show an interest in the new culture, have good communication skills, a desire to learn about a new society, and who have the selfconfidence to deal with cross-cultural adjustments. She suggested the successful sojourner will balance the values and expectations of the traveler's homeland, the host nation, and their own personal values and beliefs.

Craven (1994) then examined common shock experiences of international sojourners, namely: (a) culture, (b) language, (c) self-discovery, and (d) role. She defined culture shock as "the total of the stresses and strains that come from trying to function 
effectively in a foreign environment where the cultural norms and cues are different from those at home” (p. 438). Those experiencing culture shock often follow the U-curve experience (Kealey, 1978) in which comfort and enthusiasm for the host nation dip as the sojourner is submerged in a foreign culture, but slowly rise as the sojourner becomes more familiar and comfortable with the new environment. Language shock referred to not only learning a new language, but also the nuances and colloquialisms in a new country, even if its citizens speak the same language as the sojourner. Shock of self-discovery develops from realizing the values and beliefs practiced in a new culture, meshing them with those from the sojourners' homeland, and discovering a new understanding of personal experience.

Craven (1994) stated role shock is the most critical shock in cross-cultural adjustment. Role shock stems from a difference between role expectation by the sojourner and role definition as prescribed by host nationals. She stated communication is vital to reducing the impact of role shock and suggested cross-cultural preparation as an appropriate means to combat it. Before cross-cultural travel, individuals should focus on the understanding the four determinants of cross-cultural effectiveness as listed above, as specifically related to the expected role in the new culture, to decrease the depth of Ucurve adjustment. Cross-cultural adjustment training should continue once the sojourner arrives in the host nation to continue to minimize cross-cultural adjustment.

In summary, a handful of researchers have examined experiences of international student-athletes. Ridinger (1998) and Ridinger and Pasore (2000a) remain the only researchers to specifically look at the adjustment levels of international student-athletes when transitioning to a United States university, although Bale (1987) also examined 
motivations behind migration decisions. Overall, the work of Bale and Ridinger, among others, suggests international student-athletes appear fairly well adjusted in their college experience. Further research is warranted in this realm, however, because much has changed in the college athletics landscape since Bale’s work over 20 years ago, while Ridinger's studies used a sample of 16 international student-athletes from a single institution which somewhat limits the generalizability of the findings. The literature does suggest differences do exist between international student-athletes who have successful experiences in the United States and their domestic student-athlete teammates.

\section{Summary of Literature Review}

In order to examine differences in perspectives of international student-athletes regarding the purpose of collegiate sport in the United States, and to investigate differences in their ability to adjust to college as compared to domestic student-athletes, it is important to understand several background and antecedent issues. The purpose of this literature review was to survey prior research delving into many of those critical issues. The organization of this review was comprised of basically four areas.

The first area examined in this research was the idea of cross-cultural adjustment. Several researchers, such as Adler (1975) and Church (1982), have examined the phenomenon of the cross-cultural sojourner; someone who typically endures a series of adjustment processes, often referred to as the U-curve of adjustment, when assimilating into a new environment. Some researchers, such as Befus (1988), suggested pre-sojourn preparation can alleviate some of the stresses expected from U-curve adjustment. International student-athletes fall under the category of cross-cultural sojourners and 
should be expected to cope with several of the attributes depicted in the cross-cultural adjustment literature.

A second area examined in this literature review regards the differences in national sport policy between the school-based system employed in the United States, and the club-based system found in most other areas of the world. As pointed out by researchers such as Green and Oakley (2001) and Harvey, Beamish, and Defrance (1993), national sport policy develops differently in various countries, depending on the history, politics, and social climate of certain nations. Such differences can manifest themselves in a variety of ways among the youth who participate in sport in those nations, the affects of which are not entirely known. Enough evidences exists, as presented in this study, to warrant investigation into the ways in which student-athletes from various cultures view the purpose of sport in the United States.

A third area of review in the literature examined how athletes transition from one level of sport to another, and how that transition might result in differing perspectives of the purpose of sport. Green (2005) suggested athletes begin participation at a young age at the mass-participation level, then work their way up to the elite sport level of competition. Researchers such as Chambliss (1989), Pearson and Petitpas (1990), and Riemer, Beal, and Schroeder (2000) suggested that as athletes transition from one level to another, they endure various psychological adjustments and often have a changed perception of sport participation. International student-athletes who move not only from one country to another, but also from a club-system to a school based-system could be expected to have difference perspectives regarding sport participation from domestic student-athletes who do not transit to another country or sport system. Duda (1989) 
developed an instrument to gauge the purpose of sport participation, which can help detect differences in how subgroups perceive sport. Her instrument was used in several studies within this review and was utilized in the current study.

Finally, this literature review examined several studies which investigate adjustment to college for several different groups: (a) domestic students, (b) international students, (c) international students, and finally (d) international student-athletes. All four groups have demonstrated different abilities and deficiencies when it comes to adjusting to college. Examining such differences provides important insight. Through years of research, Baker and Siryk (1989), developed an instrument used in many studies, including this one, to gauge student adjustment to college. In particular, Ridinger (1998) developed a study which provided an excellent model for the current study. In Ridinger's (1998) work, the four subgroups of college students mentioned above were administered Baker and Siryk’s (1989) instrument, revealing key differences between groups. Such results provide excellent justification for the current study. 


\section{CHAPTER 3}

\section{METHODOLOGY}

The purpose of this chapter is to describe and elaborate on the methods used to investigate the research questions posed in Chapter 1. The methodology depicts: (a) the participants used as a sample in this study, (b) the instruments used to collect data, (c) the definitions of terms used in the data collection process, (d) the data collection itself, and (e) how the data were analyzed.

\section{Purpose of the Study}

This study had several purposes. The first purpose of this study was to assess whether international student-athletes demonstrated different abilities to adjust to college as compared to domestic student-athletes. Next, the study examined whether international student-athletes viewed the purpose of collegiate sport differently than student-athletes from the United States. Third, this study examined whether perception of purpose of collegiate sport can predict both domestic and international student-athletes' ability to adjust to college.

\section{Research Questions}

This study contained three primary research questions: (a) Do international student-athletes adjust better to college than domestic student-athletes?, (b) Do international student-athletes view the purpose of collegiate sport differently than domestic student athletes?, and (c) Do student-athletes' perspectives of the purpose of 
collegiate sport affect their adjustment to college? In order to answer these three primary questions, additional sub-questions are posed for each primary question. The first primary research question is divided into two sub-questions, the second primary research questions is divided into seven sub-questions, and the third primary questions in divided into two sub-questions. Those sub-questions are listed below:

1a. Are there significant differences between international student-athletes and domestic student-athletes in their ability to socially adjust to college?

1b. Are there significant differences between international student-athletes and domestic student-athletes in their institutional attachment to their United States college?

2a. Are there significant differences between international student-athletes and domestic student-athletes in their view of the purpose of sport as a means of mastery and cooperation of athletic skill?

2b. Are there significant differences between international student-athletes and domestic student-athletes in their view of the purpose of sport as leading to a physically active lifestyle?

2c. Are there significant differences between international student-athletes and domestic student-athletes in their view of the purpose of sport as helping an individual become a good citizen?

2d. Are there significant differences between international student-athletes and domestic student-athletes in their view of the purpose of sport as making an individual more competitive? 
2e. Are there significant differences between international student-athletes and domestic student-athletes in their view of the purpose of sport as leading to a high status career?

2f. Are there significant differences between international student-athletes and domestic student-athletes in their view of the purpose of sport as enhancing selfesteem?

2g. Are there significant differences between international student-athletes and domestic student-athletes in their view of the purpose of sport elevating social status and helping individuals get ahead?

3a. Do purpose of sport factors help predict social adjustment for either international or domestic student-athletes?

3b. Do purpose of sport factors help predict institutional attachment for either international or domestic student-athletes?

\section{Participants}

The population for this study consisted of both domestic and international student-athletes attending NCAA Division I universities. After discussing data collection methods with other sport administration faculty and athletics department administrators, a decision was made to work through the coordinators of a program called CHAMPS/Life skills. The CHAMPS/Life Skills program was initiated by the NCAA in 1991. Schools participating in the initiative develop programs offering support and services to assist student-athletes. International student-athletes are one of the primary targets for CHAMPS/Life Skills programs. 
To acquire an adequate sample from the population, CHAMPS/Life Skills coordinators at numerous NCAA Division I schools were contacted via the CHAMPS/Life Skills listserv and asked for their assistance in collection of data. Division I schools were chosen for several reasons. Of 466 CHAMPS/Life Skills programs in place at NCAA schools in 2004, 300 of them were located at Division I institutions (NCAA, 2004). In addition to having more programs, Division I schools typically have larger athletic department staffs than Division II or Division III schools and therefore were more likely to have staff members whose primary responsibility was the CHAMPS/Life Skills program. Division II and Division III schools were more likely to have CHAMPS/Life Skills programs administered by staff members who also had other responsibilities in the department. It was felt staff members who had fewer duties within their department on which to concentrate would be able to dedicate more energy in helping to collect data for this research project.

In addition, the majority of international student-athletes in the United States attended NCAA Division I universities. During the 2004-05 academic year, nearly 70\% of the international student-athletes participating in NCAA competition were doing so at the Division I level (NCAA, 2006b). In fact, according to that NCAA report, only 2,305 student-athletes among the 79,869 total competing at NCAA Division II schools were non-resident aliens (2.9\%) and only 1,050 student-athletes among the 151,059 NCAA Division III total were non-resident aliens (0.7\%). This greater number of potential participants also meant selected Division I schools would have, on average, a greater concentration of international student-athletes as compared to schools in other divisions. 
Schools which had greater concentrations of international student-athletes allowed adequate amounts of data to be collected from a relatively smaller pool of universities.

To identify participants, a request (see Appendex A) was sent via e-mail listserv to CHAMPS/Life Skills coordinators at Division I universities across the country. Because no coordinator responded to either the first or second e-mail request for help, phone calls were made to CHAMPS/Life Skills coordinators, soliciting their participation. Initially, 22 CHAMPS/Life Skills coordinators were solicited via phone and 15 agreed to participate in the study. These coordinators were selected based on a need to collect data from purposive clusters of Division I institutions. According to Bale (1991) certain factors influenced international student-athlete experience at their United States college such as: (a) school size, (b) size of the community within which school was located, and (c) geographic location of school. Schools selected for this study had nearly equal representation between enrollments of under the NCAA Division I average of 9,582 (NCAA, 2006b) students $(n=7)$ and over 9,582 students $(n=8)$. The sample contained eight schools $(n=8)$ located in communities with a population under 300,000 and seven schools $(n=7)$ located in communities with a population over 300,000. Finally, of the 15 schools selected for this study, four $(n=4)$ were located in the eastern third of the United States, six $(n=6)$ were from the midwest, and five $(n=5)$ were located in the western third of the United States.

These 15 schools had a total of 464 international student-athletes listed on their athletics websites. For each international student-athlete listed, one domestic studentathlete from the same sport was also selected at each institution. Some sports had more international student-athletes than domestic student-athletes on their roster, meaning 
slightly fewer than 464 domestic student-athletes were chosen for the sample. In total, 886 student-athletes were selected to be participants in the study. According to NCAA data (NCAA, 2006b), 81,312 student-athletes were enrolled at NCAA division I schools during the 2004-05 school year. According to Salant and Dillman (1994), when a population contains between 50,000 and 100,000 people, a sample size of 245 is necessary to achieve less than five percent sampling error rate, if the population is fairly evenly split on the characteristic of interest, which in this study was domestic or international student-athlete status. A 28\% response rate would be necessary based on the sample size of this study to receive 245 responses. The only other prior study comparing college adjustment between international and domestic student-athletes received between 32 and 36 \% response rates (Ridinger, 1998) from domestic and international studentathletes.

To conduct this study, two multivariate analyses of variance (MANOVA) were performed. These two MANOVAs provided answers to the first nine research questions, which focused on significant differences between international and domestic studentathletes’ feelings of social adjustment and institutional attachment to college and their perception of the purpose of collegiate sport. Tabachnick and Fidell (2001) suggested use of a sample size which has a greater number of participants per cell than dependent variables in the study for use of MANOVA. In this particular study, the first MANOVA had two dependent variables, namely social adjustment scores and institutional attachment scores, while the second MANOVA had seven dependent variables, namely the scores from the seven purpose of sport factors. Thus, at least eight participants per cell were necessary. In addition, Mardia (1971) suggested a minimum of 20 participant 
scores per cell in tests of MANOVA. In the current study, there was one independent variable (student-athlete status) in all MANOVAs, with two dependent variables in the first test (social adjustment and institutional attachment) and seven dependent variables in the second test (seven purpose of sport factors). Thus, the greatest number of cells in the two MANOVAs was seven, meaning 140 (7 x 20) total participants were needed, half of whom were international student-athletes and half of whom were domestic studentathletes.

To answer the final two research questions in the study, namely could purpose of sport scores help predict social adjustment to college and institutional attachment, four multiple regression analyses were performed. Tabachnick and Fidell (2001) recommended a sample size of $N>50+8 m$, where $m$ is the number of independent variables in a multiple correlation or $N>104+m$ for testing individual predictor variables. Both equations are based on detection of medium effect size. For this study, seven independent variables were measured, meaning a minimum sample size of 106 international and 106 domestic student-athletes were necessary for the multiple correlation equation and a minimum of 111 international and 111 domestic studentathletes were necessary to test the effect of the individual independent variables.

\section{Instrumentation}

Two different instruments were used to collect data in this study. The first was an abbreviated form of the Student Adaptation to College Questionnaire (SACQ) developed by Baker and Siryk (1989). The full SACQ contains 67 items which measure how well students adjust to college. This instrument has been used in numerous studies assessing student adjustment to college, including adjustment by non-United States university 
students (Byers \& Goossens, 2002) and by domestic and international student-athletes (Ridinger \& Pastore, 2000a). Baker and Siryk concluded student adaptation to college is a multi-faceted construct. They determined college adaptation could be measured under four different dimensions, thus the SACQ contains four subscales: (a) academic adjustment, (b) social adjustment, (c) personal-emotional adjustment, and (d) institutional attachment. When completing the SACQ, participants rate items in these four subscales on 9-point, Likert-type scales anchored by the phrases "applies very closely to me" to “doesn’t apply to me at all.”

\section{Student Adaptation to College Questionnaire}

Numerous researchers have employed the SACQ to assess student adjustment to college. The instrument has been shown to have high reliability and validity. Between 1980 and 1985, Baker and Siryk conducted seven different studies at two different colleges using the SACQ, and in all seven studies, coefficient alpha scores for all four subscales ranged from .77 to .91. Several other researchers including Harris (1988), Caldwell (1987), McGown (1987), and Gerdes (1986) have used the SACQ at various universities and achieved similarly high coefficient alpha inter-reliability scores for the SACQ subscales.

While all four subscales of the SACQ measure important aspects of college adjustment, and previous researchers suggest the potential for finding significant results from measuring all four subscales (Bale 1991; Ridinger, 1998; Ridinger and Pastore 2000a), a decision was made to only measure two of the subscales in this study. This decision was based upon several justifications. First, previous research (Ridinger, 1998) has shown collecting data from international and domestic student-athletes using lengthy 
surveys yields extremely poor response rates. Dillman (2000) has also suggested shorter instruments can yield greater response rates.

In addition, previous researchers (Kaczmarek, Matlock, Merta, Ames, \& Ross, 1994) administering the SACQ to international and domestic student populations, found no significant differences on the academic and personal-emotional adjustment scales between the two groups, but did find international students scored significantly lower on social and institutional attachment scales. They suggested this difference was attributable in large part to international students' lack of participation in campus activities and leadership roles. Because international student-athletes are involved in extra nonacademic activities on campus, their scores on the scales of social adjustment and institutional attachment could very well be different from those of other international students. Thus, those two scales would appear to measure important factors in international student-athlete adjustment to college.

Finally, only two of the SACQ subscales specifically addressed the research subquestions. Thus a decision was made to use only the subscales of social adjustment, which contained 20 items, and institutional-attachment, which contained seven unique items and seven items shared with social adjustment. The elimination of the other two subscales reduced 41 items from the original SACQ. Previous researchers (Hannum \& Dvorak, 2004) have successfully used just one subscale from the full SACQ to measure certain types of adjustment in college students.

\section{Purpose of Sport Questionnaire}

The second instrument used in the study was the Purpose of Sport Questionnaire, developed by Duda (1989). The original Purpose of Sport Questionnaire, which contains 
46 items, was based in part upon the Purpose of Schooling Questionnaire (Nicholls, Patashnick, \& Nolen, 1985; Thorkildsen, 1988) and in part on other literature dealing with the values and benefits of sport participation. Duda's initial study revealed participants held several different views regarding what sport should teach or do for participants. These various reasons helped explain a variety of motivations for sport participation among youth in Duda's study. Duda also ran responses from her questionnaire through a factor analysis, and found seven main factors emerged. These seven factors were labeled: (a) mastery/cooperation, (b) physically active lifestyle, (c) good citizen, (d) competitiveness, (e) high-status career, (f) enhance self-esteem, and (g) social status/getting ahead (Duda, 1989). All factors produced strong levels of internal consistency in her study, with Cronbach's alpha coefficients ranging from .75 to .83 (Duda, 1989). White (1995) used the Purpose of Sport Questionnaire with college students and also found strong levels of internal consistency among the same seven factors, with Cronbach’s alpha coefficient scores ranging from .79 to .87 .

In the original Purpose of Sport Questionnaire, participants responded to the phrase "A very important thing sport should do is...” by rating different concluding phrases to this stem on a 5-point, Likert-type agreement scale. When run through a factor analysis, all 46 items produced factor loading scores above .40. Certain items, however, were more pure measures of factors, as indicated by their higher factor loading scores (Duda, 1989). According to Comrey and Lee (1992), items with factor loading scores of .71 or higher are considered excellent measures of a factor. Items with factor loading scores of .63 to .71 are considered very good measures of a factor and items with factor loading scores ranging from .55 to .63 are considered good measures of a factor. If a 
factor loading score of an item is below .55, it is considered a fair or poor measure of a factor. In an effort to reduce the number of items used for this study, which in turn would improve the response rate, only items producing factor loadings in the good, very good, or excellent ranges were kept.

One exception was made to accept a single item which loaded below .55. The first factor in the Purpose of Sport Questionnaire, “mastery/cooperation”, only had two items load at a level over .55. According to Tabachnick and Fidell (2001), it is hazardous to interpret a factor in a factor analysis when it is defined by only one or two items. Because of this, a third item was needed for this factor. The next highest loading factor for “mastery/cooperation”, which was "learn what was meant by teamwork” was included, despite its factor loading score of .52.

These decisions reduced the number of items kept in the scale to 31, with at least three items retained under each factor. In addition, two items were eliminated from the original Purpose of Sport Questionnaire because of their irrelevance to college studentathletes. The Purpose of Sport Questionnaire was originally designed for high school students and included the items "An important thing sport should do is help us get into the best colleges" and "An important thing sport should do is give us the chance to be friends with popular kids” but both these items were deleted from the instrument utilized in this study.

The final instrument used in this study contained 27 items from the SACQ and 29 items from the Purpose of Sport Questionnaire. In addition, participants were also asked to supply answers to 6 demographic items: (a) gender, (b) year in school, (c) native country, (d) years spent in the United States, (e) approximate cumulative grade point 
average, and (f) sport played in college. The final instrument used for this study contained 62 total items.

\section{Operational Definitions}

Operational definitions are ways in which researchers can view and measure different variables in a study. Such definitions are critical because the way in which the variables are measured will allow others to replicate the same study (Vogt, 1999). For the two MANOVAs conducted in this study, the independent variables were student-athlete status, which was labeled either international or domestic. International student-athletes are individuals enrolled at a university who are members of an intercollegiate athletic team and are citizens of a country outside of the United States (Ridinger, 1998). Domestic student-athletes are individuals enrolled at a university who are members of an intercollegiate athletic team and are citizens of the United States (Ridinger, 1998).

In addition to the two independent variables, there were two dependent variables in the first MANOVA of this study and seven dependent variables in the second MANOVA. The two dependent variables in the first MANOVA depicted student adjustment to college. The first dependent variable was social adjustment which was operationally defined as the sum of all scores for questions 1 through 20 on the SACQ (Baker \& Siryk, 1989), divided by 20. The second dependent variable was institutional attachment and was operationally defined as the sum of all scores for questions 1, 2, 6, 9, 13, 17, 18 and 21 through 27 on the SACQ, divided by 14 .

The seven dependent variables in the second MANOVA of this study, which were used to answer research questions three through nine, were the seven factor scores from the Purpose of Sport Questionnaire. Each factor score was derived from the mean score 
of all responses to the items on the Purpose of Sport Questionnaire related to each factor. The first factor, "mastery/cooperation," contained three items, questions 1,2 , and 3 . The second factor, "physically active lifestyle,” contained three items, questions 4, 5, and 6. The third factor, "good citizen,” contained six items, questions 7, 8, 9, 10, 11, and 12. The fourth factor, “competitiveness,” contained four items, questions 13, 14, 15, and 16. The fifth factor, "high status career," contained four items, questions 17, 18, 19, and 20. The sixth factor, “enhance self-esteem,” contained five items, questions 21, 22, 23, 24, and 25. The seventh factor, "social status/getting ahead,” contained four items, questions 26, 27, 28, and 29.

To answer the final two research questions regarding whether Purpose of Sport factor scores predicted social adjustment and institutional attachment, four multiple regression analyses were conducted. Each regression had seven independent variables, namely the seven mean factor scores from the Purpose of Sport Questionnaire as described above. Scores from the social adjustment and institution attachment scales on the SACQ served as the dependent variables in the multiple regressions.

\section{Data Collection}

To collect data for this study, a modified version of the Dillman (2000) method was employed. Each assisting CHAMPS/Life Skills coordinator at participating schools was contacted by telephone and was given further background about the study and what would be expected of them should they agree to participate. Approximately one week after this phone call, each participating coordinator received copies of the research instrument (see Appendix B), plus instructions for its administration (see Appendix C), and a list of student-athletes from that school selected for the study. Each coordinator was 
instructed to distribute the instrument, along with a letter from the researcher explaining the study (see Appendix D) to all student-athletes on the list. The coordinator instructed the student-athletes to complete the survey, seal it in an envelope which was also provided, and return it to the coordinator. After two weeks, all participating CHAMPS/Life Skills coordinators were sent an e-mail (see Appendix E), which they were asked to forward to all student-athletes who were taking part in the study. This email served as a thank you to all participants who completed the survey and served as a reminder to those who had not completed the survey to do so. After approximately four weeks from the initial distribution, the CHAMPS/Life Skills coordinator mailed all completed surveys back to the researcher.

\section{Data Analysis}

Frequencies, means, and standard deviations were calculated and reported from the returned instruments. Reliability coefficients as measured by Chronbach’s alpha scores were calculated for the two subscales of the SACQ, and for the seven factors of the Purpose of sport questionnaire.

To answer the first nine research questions, multivariate analysis of variance (MANOVA) tests were used to detect significant differences in group means. The first two research questions asked whether significant differences existed for social adjustment to college between international and domestic student-athletes and for institution attachment between international and domestic student-athletes. Scores from the SACQ social adjustment and institution attachment subscales served as the dependent variables for these first two questions. Domestic or international student-athlete status served as the independent variables. For research questions three through nine, mean scores from the 
Purpose of Sport Questionnaire factors were used as dependent variables in the MANOVA. Domestic or international student-athlete status was again the independent variable.

When conducting tests of MANOVA, researchers must ensure the data used meet certain assumptions; namely independent observations, homogeneity of variance, and normality of distribution. The method utilized to conduct data collection ensured independent observations because each participant individually completed and returned a single survey. A Levene's test was used to test homogeneity of variance, while histograms of the data were utilized to measure normal distribution (Tabachnick \& Fidell, 2001). A within-cell correlation matrix was also produced from the data for each MANOVA to check for multicollinearity of the dependent variables (Tabachnick \& Fidell, 2001).

To answer the final two research questions, namely do student-athletes’ perceptions of the purpose of sport significantly predict social adjustment to college or institutional attachment, four multiple regressions were employed. In all four regressions, the independent variables consisted of the seven Purpose of Sport Questionnaire factor scores. In the first two regressions, international and domestic student-athlete scores for social adjustment to college served as the dependent variables, while in the third and fourth regressions, international and domestic student-athlete scores for institutional attachment served as the dependent variables.

Assumptions for multiple regressions include: (a) independence of scores, (b) normal distribution, (c) homoscedasticity among dependant variables, and (d) linearity among the dependant variables. It is also advisable to screen for multicollinearity among 
the independent variables (Tabachnick \& Fidell, 2001). Again, individual completion of a single survey by all participants ensured independence of scores. Scatterplot diagrams were developed for all data used in the multiple regression analyses to test for normal distribution, homoscedasticity, and linearity. Collinearity statistics were produced and examined to screen for extreme multicollinearity among the purpose of sport factor scores.

\section{Summary of Methodology}

In summary, the sample for this study included international and domestic student-athletes from 15 NCAA Division I institutions. Participating schools were selected using a stratified random selection process. Student-athletes were contacted through the use of a mediating person, namely the CHAMPS/Life Skills coordinator at each participating school. All participants were asked to complete a survey containing a total of 56 survey items and six demographic variable questions. Data collection took place through use of a modified version of the Dillman (2000) method. Responses were collected and analyzed through two different statistical methods, MANOVA and multiple regression, in order to answer the 11 research questions posed in this study. Data were screened for the appropriate assumptions for use in the statistical tests employed for this study. Results of data collection are presented in the following chapter. 


\section{CHAPTER IV}

\section{RESULTS}

The purpose of this chapter is to report the results of this study. This chapter is divided into the following sections: (a) demographic variables, (b) instrument reliability, (c) results from the research questions, and (d) a summary. This study had several purposes. The first purpose of this study was to assess whether international studentathletes demonstrate different abilities to adjust to college as compared to domestic student-athletes. Next, the study examined whether international student-athletes viewed the purpose of collegiate sport differently than student-athletes from the United States. Third, the study examined whether perception of purpose of collegiate sport can predict both domestic and international student-athletes' ability to socially adjust to college their institutional attachment to their school.

\section{Demographic Variables}

The following is a list of the important demographic variables in this study. Fifteen schools initially agreed to participate in the study, but ultimately only 11 schools followed through with data collection by returning surveys. An effort was made to collect an equal amount of data from schools with large enrollments and schools with small enrollments to obtain a representative sample of NCAA Division I college athletes. In the most recent NCAA report on graduation rates for division I institutions, the average enrollment during the 2003-04 school year was reported at 9,582 students (NCAA, 
2006b). Of the 11 schools involved in this study, six had enrollments greater than 9,582 students, while five had an enrollment of fewer than 9,582 students.

To further ensure the sample was representative of the population, studentathletes were selected from schools in geographically diverse locations and categorized as either attending rural and urban campuses. Bale $(1987,1991)$ reported prior to 1990 , many international student-athletes attended schools in rural locations. However, in the last two decades, international student-athletes have been recruited by schools of all sizes and in all locations (NCAA, 2006b). In this study, six of the participating schools were located in urban communities, those with populations greater than 300,000 people, while five schools were located in rural communities, or areas with populations below 300,000 people. Three of the participating schools were located in the eastern third of the United States, four of the schools were located in the Midwest, and four of the participating schools were located in the western third of the United States.

In total, 649 surveys were sent to the 11 participating schools, with 338 of those surveys directed towards international student-athletes and 311 surveys directed toward domestic student-athletes. Some participating schools had more international studentathletes than domestic student-athletes participating on certain teams, resulting in slightly unequal participant numbers for the two groups. Of those surveys, 13 were returned blank because the student-athlete they were addressed to was no longer enrolled at the school. Thus the final sample size for this study was $636(N=636)$ student-athletes. Of that sample, 284 surveys were returned for a response rate of $45.3 \%$. Returned responses came from 110 domestic student-athletes and 174 international student-athletes, while four respondents did not indicate whether they were international or domestic. 
All student-athletes were asked to indicate what they considered to be their native country. Respondents named 50 different countries. The nations represented most often, outside the United States, were Canada with 49 (28.2\%) respondents, Germany with 11 (6.3\%) respondents, Australia and Sweeden with eight (4.6\%) respondents each, Norway with seven (4.0\%) respondents, England and Poland with six (3.5\%) respondents each, and Jamaica, the Netherlands, South Africa, and Serbia with four (2.3\%) respondents each. A complete list of frequencies can be found in Table 1.

In the last study conducted by the NCAA (1996) on international student-athletes, which covered the 1995-96 school year, the largest percentage of international studentathletes came from Canada (28.4\%), followed by England (6.3\%), Sweden (5.8\%), Australia (3.2\%), Germany (2.6\%), South Africa (2.5\%), Jamaica (2.3\%), Ireland (2.2\%), Brazil (2.1\%), and Norway (1.8\%). Eight of the top 11 countries (outside the United States) with the highest representation in the current study were also among the top 10 in the NCAA study, with Canada comprising by far the largest single-nation percentage in both studies at just over 28 percent. Because participants in this study were selected via a clustered random selection process and because of the demographic data outlined above, the sample in this study appeared quite representative of the population. 
Table 1.

Native Country of Respondents $(n=174)$

\begin{tabular}{|c|c|c|}
\hline Native Country & No. & $\%$ \\
\hline Canada & 49 & 28.2 \\
\hline Germany & 11 & 6.3 \\
\hline Australia & 8 & 4.6 \\
\hline Sweden & 8 & 4.6 \\
\hline Norway & 7 & 4.0 \\
\hline England & 6 & 3.5 \\
\hline Poland & 6 & 3.5 \\
\hline Jamaica & 4 & 2.3 \\
\hline Netherlands & 4 & 2.3 \\
\hline South Africa & 4 & 2.3 \\
\hline Serbia & 4 & 2.3 \\
\hline Austria & 3 & 1.7 \\
\hline Cameroon & 3 & 1.7 \\
\hline France & 3 & 1.7 \\
\hline Italy & 3 & 1.7 \\
\hline Mexico & 3 & 1.7 \\
\hline Russia & 3 & 1.7 \\
\hline Slovenia & 3 & 1.7 \\
\hline Ukraine & 3 & 1.7 \\
\hline China & 2 & 1.2 \\
\hline Croatia & 2 & 1.2 \\
\hline Ireland & 2 & 1.2 \\
\hline
\end{tabular}




\begin{tabular}{|c|c|c|}
\hline Kenya & 2 & 1.2 \\
\hline Latvia & 2 & 1.2 \\
\hline Puerto Rico & 2 & 1.2 \\
\hline Slovakia & 2 & 1.2 \\
\hline Trinidad and Tobago & 2 & 1.2 \\
\hline Venezuela & 2 & 1.2 \\
\hline Argentina & 1 & 0.6 \\
\hline Barbados & 1 & 0.6 \\
\hline Colombia & 1 & 0.6 \\
\hline Dominican Republic & 1 & 0.6 \\
\hline Ecuador & 1 & 0.6 \\
\hline Ghana & 1 & 0.6 \\
\hline Honduras & 1 & 0.6 \\
\hline Ivory Coast & 1 & 0.6 \\
\hline Israel & 1 & 0.6 \\
\hline Japan & 1 & 0.6 \\
\hline Lebanon & 1 & 0.6 \\
\hline Liberia & 1 & 0.6 \\
\hline Lithuania & 1 & 0.6 \\
\hline Mali & 1 & 0.6 \\
\hline New Zealand & 1 & 0.6 \\
\hline Singapore & 1 & 0.6 \\
\hline Somalia & 1 & 0.6 \\
\hline Tonga & 1 & 0.6 \\
\hline Tunisia & 1 & \\
\hline
\end{tabular}




\begin{tabular}{lcc} 
Uruguay & 1 & 0.6 \\
Zimbabwe & 1 & 0.6 \\
\hline
\end{tabular}


Other demographic information collected in the current study included (a) gender, (b) year in school, (c) number of years spent in the United States, (d) approximate cumulative grade point average, and (e) sport played. Among respondents, 127 (44.1\%) were male and 157 (54.5\%) were female, while four did not indicate gender. Among respondents indicating both gender and international or domestic student-athlete status, 43 were domestic males while 67 were domestic females. Among international studentathletes, 82 were male and 90 were female.

Of all respondents, 82 (28.5\%) were in their freshman year, 69 (24.0\%) were in their sophomore year, 77 (26.7\%) were in their junior year, 45 (15.6\%) were in their senior year, five (1.7\%) indicated they were graduate students, and 10 (3.5\%) did not indicate their year in school. Regarding years spent in the United States, for international student-athletes the mean was 2.9 years, while for domestic student-athletes the mean was 20.1 years. Among all respondents, the mean cumulative grade point average was 3.14, with 77 (26.7\%) reporting a GPA below 3.00, 117 (40.6\%) reporting a GPA between 3.00 and 3.49, and 80 (27.8\%) reporting a cumulative GPA of 3.50 or higher. Fourteen (4.9\%) respondents did not offer their GPA, while five student-athletes reported a perfect 4.00 GPA. The mean GPA scores for domestic and international student-athletes in this study were identical at 3.14.

Respondents reported participating in 17 different sports, seven of which were either exclusively male (cross country, football, and ice hockey) or exclusively female (field hockey, gymnastics, softball, and water polo). The most responses came from tennis players at 66 (22.9\%), followed by soccer players at 37 (12.8\%), swimmers at 33 (11.5\%), basketball players at 27 (9.4\%), and track and field competitors at $23(8.0 \%)$. 
Three student-athletes reported participating in two different sports at their school while five did not indicate their sport played. A complete list of sport frequencies can be found in Table 2. The breakdown of sports played in the current study closely mirrored the latest NCAA national participation report (NCAA, 2006b). In that document, the top six sports with the highest concentration of non-resident aliens were tennis (20.3\%), track and field (11.3\%), soccer (10.2\%), basketball (7.7\%), golf (6.7\%), and swimming (6.4\%), meaning the top five represented sports in the current study were among the top six in the NCAA report. 
Table 2.

Sports Played by Respondents $(n=280)$

\begin{tabular}{|c|c|c|}
\hline Sport & No. & $\%$ \\
\hline Tennis & 66 & 22.9 \\
\hline Soccer & 37 & 12.8 \\
\hline Swimming & 33 & 11.5 \\
\hline Basketball & 27 & 9.4 \\
\hline Track and Field & 23 & 8.0 \\
\hline Volleyball & 22 & 7.6 \\
\hline Golf & 15 & 5.2 \\
\hline Rowing & 14 & 4.9 \\
\hline Skiing & 12 & 4.2 \\
\hline Ice Hockey & 8 & 2.8 \\
\hline No Sport & 5 & 1.7 \\
\hline Football & 4 & 1.4 \\
\hline Gymnastics & 4 & 1.4 \\
\hline Softball & 4 & 1.4 \\
\hline Cross Country & 3 & 1.0 \\
\hline Lacrosse & 3 & 1.0 \\
\hline Water Polo & 3 & 1.0 \\
\hline Multiple Sports & 3 & 1.1 \\
\hline Field Hockey & 2 & 0.7 \\
\hline
\end{tabular}




\section{Instrument Reliability}

This study examined how well international and domestic student-athletes socially adjusted to college and how attached they were to their college institution. It also investigated student-athletes’ perceived perceptions of the purpose of sport. To determine these relationships and perceptions, all student-athletes in this study were administered a one page (two-sided) paper-and-pencil survey, which was completed in private and returned in a sealed envelope.

\section{Field Test of Instrument}

Portions of two instruments used extensively in the literature, the Student Adaptation to College Questionnaire (SACQ; Baker \& Siryk, 1989) and the Purpose of Sport Questionnaire (Duda, 1989), were combined into one instrument for the current study. Both the SACQ and the Purpose of Sport Questionnaire have stood up to numerous tests of reliability and validity (Baker \& Siryk, 1986; 1989; Caldwell, 1987; Carpenter \& Yates, 1997; Duda, 1989; Gerdes, 1986; Harris, 1988; McGowan, 1987; Ridinger, 1998; Treasure, Carpenter, \& Power, 2000; White, 1995). Given the study was to be administered to many international student-athletes whose native language was not English, a decision was made to field test the combined instrument to ensure the instructions and questions were easily comprehended, as well as to gauge the amount of time needed to complete the survey. Five international student-athletes at an NCAA division II school completed the instrument, then filled out a short questionnaire rating the instrument. All respondents indicated the survey was easy to understand, looked professional, and took between five and ten minutes to complete. The only criticisms of the instrument dealt with issues beyond the scope of this particular study, although 
comments indicated international student-athletes were interested in having their voice heard on issues of adjustment to college and to living in the United States.

\section{Scale Reliability and Consistency}

Once all the data from the study were collected, all nine scales in the instrument (two from the SACQ and seven from the Purpose of Sport Questionnaire) were assessed for reliability through an analysis of inter-item consistency. Cronbach's alpha coefficients were derived for all scales. From the SACQ, the social adjustment scale produced a Cronbach’s alpha coefficient of .864 and the institutional attachment scale produced a Cronbach’s alpha coefficient of .837, both exceeding a minimum value of .70 as suggested by Nunnally and Bernstein (1994).

Among the Purpose of Sport scales, five produced Cronbach's alpha scores between .710 and .855, also meeting Nunnally and Bernstein’s (1994) minimum. The other two scales, namely Mastery/Cooperation and High Status, produced Cronbach’s alpha coefficients of .591 and .585, respectively. The elimination of one item in each scale significantly improved inter-item consistency, so a decision was made to drop those items from the scales. In the Mastery/Cooperation scale, the elimination of the item " $\mathrm{A}$ very important thing college sports should do is teach us what is meant by teamwork," improved the consistency rating to .626. In the High Status scale, the elimination of the item “A very importing thing college sports should do is give us a chance to be a professional athlete,” improved the consistency coefficient to .666. While the changes in those two scales still did not increase the Cronbach’s alpha coefficient above .70, Aiken (2000) has stated when gauging significant differences between group means, scale reliability scores between .60 and .70 are acceptable. Results from this study regarding 
these scales should be interpreted with caution, however, because of the aforementioned changes. A complete listing of the Cronbach's alpha scores for all scales can be found in Tables 3 and 4. 
Table 3.

Inter-item Consistency Ratings of SACQ Scales

\begin{tabular}{lcc} 
Scale & Items & Alpha \\
\hline Social Adjustment & 20 & 0.864 \\
Institutional Attachment & 14 & 0.837 \\
\hline
\end{tabular}

Table 4.

Inter-item Consistency Ratings of Purpose of Sport Questionnaire Scales

\begin{tabular}{lcc}
\hline Scale & Items & Alpha \\
\hline Mastery and Cooperation & $2^{*}$ & 0.626 \\
Physically Active & 3 & 0.851 \\
Good Citizen & 6 & 0.728 \\
Competitiveness & 4 & 0.711 \\
High Status & $3 *$ & 0.666 \\
Self-Esteem & 5 & 0.749 \\
Social Status & 4 & 0.825 \\
\hline
\end{tabular}

*one item removed to improve factor measurability 


\section{Mean Scores and Correlation Matrices of Instrument Factors}

The SACQ portion of the instrument had 28 items which loaded on two factors. Respondents rated the items on an application scale with the anchor one representing an item which did not apply at all to the respondent and the anchor nine representing an item which strongly applied to the respondent. Mean scores for the two SACQ factors are listed in Table 5. In addition, the Purpose of Sport Questionnaire portion of the instrument contained 29 items which loaded on seven factors. Respondents rated those items on an agreement scale anchored by one, representing strong disagreement, and five, representing strong agreement. Mean scores for the seven Purpose of Sport Questionnaire factors are listed in Table 6. A correlation matrix depicting the relationship between SACQ factors can be found in Table 7, while a correlation matrix depicting the relationship between Purpose of Sport Questionnaire factors can be found in Table 8. 
Table 5.

Mean Scores and Standard Deviations on SACQ

\begin{tabular}{lcc} 
Scale & Domestic & International \\
\hline Social Adjustment & $7.17(0.972)$ & $6.71(1.059)$ \\
Institutional Attachment & $7.44(1.126)$ & $7.13(1.060)$ \\
\hline
\end{tabular}

Note. Mean scores based on 9-point Likert-type scale

Table 6.

Mean Scores and Standard Deviations on Purpose of Sport Questionnaire

\begin{tabular}{lcc} 
Scale & Domestic & International \\
\hline Mastery and Cooperation & $4.10(0.842)$ & $4.24(0.788)$ \\
Physically Active & $4.19(0.869)$ & $4.11(0.894)$ \\
Good Citizen & $4.26(0.592)$ & $4.13(0.585)$ \\
Competitiveness & $4.03(0.727)$ & $3.77(0.772)$ \\
High Status & $4.20(0.675)$ & $4.21(0.718)$ \\
Self-Esteem & $4.35(0.526)$ & $4.34(0.610)$ \\
Social Status & $2.74(1.035)$ & $2.82(1.018)$ \\
\hline
\end{tabular}

Note. Mean scores based on 5-point Likert-type scale 
Table 7.

Correlation Matrix for SACQ Factors $(N=288)$

$\begin{array}{lll}\text { Variable } & 1 & 2\end{array}$

1. Social Adjustment $\quad$-- $\quad 0.84^{*}$

2. Institutional Attachment

*Correlation is significant at the 0.01 level

Table 8.

Correlation Matrix for Purpose of Sport Factors $(N=288)$

\begin{tabular}{lccccccc} 
Variable & 1 & 2 & 3 & 4 & 5 & 6 & 7 \\
1. Mastery/Cooperation & -- & $0.30^{*}$ & $0.23^{*}$ & 0.00 & $0.23^{*}$ & $0.25^{*}$ & 0.03 \\
2. Physically active & & -- & $0.47^{*}$ & $0.35^{*}$ & $0.33^{*}$ & $0.46^{*}$ & $0.31^{*}$ \\
3. Good citizenship & & -- & $0.46^{*}$ & $0.43^{*}$ & $0.50^{*}$ & $0.27^{*}$ \\
4. Competitiveness & & & -- & $0.36^{*}$ & $0.46^{*}$ & $0.43^{*}$ \\
5. High status & & & & -- & $0.41^{*}$ & $0.33^{*}$ \\
6. Self-esteem & & & & & & -- & $0.33^{*}$ \\
7. High social status & & & & & & & \\
\hline *Correlation is significant at the 0.01 level
\end{tabular}




\section{Results of Research Questions}

To answer the research questions posed in this study, two types of statistical tests were utilized to analyze the data. Two separate multivariate analyses of variance (MANOVAs) were run on the data to answer the first nine research questions. The MANOVA tests were utilized to compare group mean scores between domestic and international student-athletes. To answer the final two research questions in this study, four multiple regression analyses were used. These multiple regression analyses illustrated any predictive ability of purpose of sport factor scores in determining social adjustment to college and institutional attachment for both domestic and international student-athletes.

\section{Assumptions of MANOVA}

Before a MANOVA test can be used, data must meet certain assumptions, namely independent observations, homogeneity of variance, and normality of distribution (Tabachnick \& Fidell, 2001). In this particular study, all observations were independent as each participant completed a single survey. A Levene's test was utilized to gauge homogeneity of variance among the dependent variables. For the SACQ variables, the Levene's test was not significant for both social adjustment and institutional attachment at the .05 level, meaning no violation of homogeneity was present. Results of a Levene's test for the Purpose of Sport factors resulted in all seven factors proving not significant at the .05 level. To determine normality of distribution, all dependent variables were placed on histograms and compared to the normal distribution curve. No abnormalities were detected. SPSS MANOVA was used to conduct all assumption tests and both MANOVA analyses. 


\section{Differences in SACQ Factors Between International and Domestic Student-Athletes}

The first two research questions posited whether significant differences existed between international student-athletes and domestic student-athletes in their ability to socially adjust to college and in their attachment to their institution. Social adjustment and institutional attachment scores served as dependent variables while domestic or international student-athlete status served as the independent variable. The MANOVA revealed a significant effect, Wilks $\Lambda=.947, F(2,281)=7.798, p=.001$. A follow-up univariate F-test revealed a statistically significant difference in both social adjustment scores $(p=.000)$ and institutional attachment scores $(p=.021)$. In both cases, domestic student-athletes reported higher scores than international student-athletes, indicating better adjustment and closer attachment for domestic student-athletes. On the 9-point Likert-type scale, domestic student-athletes reported an overall mean score on the social adjustment scale of 7.17, while international student-athletes had an overall mean score of 6.71. On the institutional attachment scale, domestic student-athletes had an overall mean score of 7.44, while international student-athletes had an overall mean score of 7.13.

Differences in Purpose of Sport Factors Between International and Domestic StudentAthletes

A second MANOVA was conducted to answer the next seven research questions, which posited whether a significant difference existed between domestic and international student-athletes in their perspective of the seven purposes of sport. These seven purposes were: (a) mastery/cooperation, (b) physically active lifestyle, (c) good citizen, (d) competitiveness, (e) high-status career, (f) enhance self-esteem, and (g) social 
status/getting ahead. Again, the purpose of sport scores served as dependent variables, while international or domestic status served as the independent variable. The MANOVA revealed a significant effect, Wilks $\Lambda=.938, F(7,276)=2.617, p=.012$. A follow-up univariate F-test was conducted to determine where significant differences existed among the specific factors. Only one factor, competitiveness $(p=.004)$, revealed a significant difference at the .05 level between international and domestic student-athletes. Mean scores for the remaining purpose of sport factors were not significantly different between international and domestic student-athletes at the .05 level. The mean score for domestic student-athletes in the competitiveness factor was 4.03, while the mean score of international student-athletes was 3.77. These results indicated domestic student-athletes felt stronger than did international student-athletes that the purpose of sports was about learning to be more competitive.

Purpose of Sport Predicting Social Adjustment and Institutional Attachment

To answer the final two research questions, four multiple regression analyses were conducted. The first two were used to detect whether perception of purpose of sport significantly predicted social adjustment for either international or domestic studentathletes. The second two multiple regression analyses determined whether any of the purpose of sport factors significantly predicted institutional attachment for either international or domestic student-athletes. Entry of Variables Using Multiple Regression

When using multiple regression analysis, several methods exist for the entry of independent variables into the equation. Standard or simultaneous regression was utilized in this particular study to determine if purpose of sport factors could predict social 
adjustment and institutional attachment scores. Because this research is exploratory in nature, with no theoretical equation suggested in the literature, hierarchal entry was not used. Meanwhile, stepwise regression was not utilized because the intent of this study was not to develop a prediction equation, but merely to detect whether the independent variables offered some ability to predict membership within the dependent variable (Tabachnick \& Fidell, 2001).

\section{Sample Size for Multiple Regression}

In order to use multiple regression tests, data must meet adequate case-to-IV ratios and certain assumptions. Green (1991) suggested a sample size of $N \geq 104+m$, where $m$ is the number of independent variables. Two of the regressions in this study had two independent variables, while the other two had seven independent variables, meaning a sample size equal or greater than 111 was recommended. The international studentathlete sample in this study was 174 , while the domestic student-athlete sample was one shy of Green's suggested minimum at 110. Other researchers, such as Shavelson (1996), have indicated a sample size with at least 10 cases per independent variable is adequate. Assumptions of Multiple Regression

According to Shavelson (1996), a multiple regression analysis must have: (a) independence among scores, (b) normal distribution among dependent variables, (c) homoscedasticity among dependant variables, and (d) linearity among the dependant variables. In addition, Tabachnick and Fidell (2001) recommend screening for multicollinearity among independent variables. All of the assumptions above were addressed in the current study. Each survey was completed individually by a single participant, ensuring independence of scores. To address normality, homoscedasticity, 
and linearity, scatterplot diagrams were developed through SPSS with predicted dependent variable scores and actual dependent variable scores used as the axises. Examination of the scatterplots revealed no problems with any of the assumptions. Collinearity statistics were produced by SPSS to test for multicollinearity among independent variables. For international student-athletes, all independent variables had a tolerance level ranging from .581 to .812. The closer the tolerance level is to 1 , the better because it means more unique predictability is added to the equation. The closer the tolerance level is to zero, the more collinearity there is among predictors (Vogt, 1999). Variance inflation factor (VIF) scores for international student-athletes ranged from 1.23 to 1.72, which fell well below the accepted standard of less than four (Fox, 1991). For domestic student-athletes, tolerance levels ranged from .564 to .847 while VIF scores fell between 1.18 and 1.77 .

\section{Purpose of Sport and Social Adjustment to College}

The 10th research question in this study posited whether purpose of sport factors explained some of the variance in social adjustment to college for either domestic or international student-athletes. Two multiple regression analysis were utilized to answer this question. The seven purpose of sport factor scores served as independent variables, while social adjustment scores served as the dependent variable. In the first regression, only international student-athlete scores were used, while in the second, only domestic student-athlete scores were used. The regression analyses revealed no statistically significant relationship between any of the purpose of sport scores and social adjustment to college for either international or domestic student-athletes. The total linear combinations of purpose of sport scores explained just over $7 \%\left(R^{2}=.071\right)$ of the 
variance among social adjustment scores for international student-athletes and just under $11 \%\left(R^{2}=.109\right)$ of the variance among social adjustment scores for domestic studentathletes.

\section{Purpose of Sport and Institutional Attachment}

The final research question in this study posited whether purpose of sport factors explained some of the variance in institutional attachment for either domestic or international student-athletes. Two multiple regression analyses were utilized to answer this question. The seven purpose of sport factor scores served as independent variables, while institutional attachment served as a dependent variable. In the first regression, only international student-athlete scores were used, while in the second, only domestic student-athlete scores were used.

The analyses revealed significant relationships between purpose of sport scores and institutional attachment for both international and domestic student-athletes. The linear combination of all purpose of sport scores explained over $18 \%\left(R^{2}=.185\right.$, adjusted $\left.R^{2}=.151, \mathrm{~F}(7,166)=5.398 ; p<.000\right)$ of the variance among institutional attachment scores for international student-athletes and nearly $14 \%\left(R^{2}=.139\right.$, adjusted $R^{2}=.080$, $\mathrm{F}(7,102)=2.352 ; p=.029)$ of the variance among institutional attachment scores for domestic student-athletes.

Good citizen $(p=.004)$, self-esteem $(p=.011)$, and social status $(p<.001)$ all significantly contributed to the prediction of institutional attachment for international student-athletes. Self-esteem $(p=.040)$ and social status $(p=.027)$ significantly contributed to the prediction of institutional attachment for domestic student-athletes. Complete regression tables can be found in Table 9 and Table 10. The standardized 
coefficients for the significant factors showed positive relationships with good citizen ( $\beta$

$=.265$ for international $)$ and self-esteem $(\beta=.234$ for international, $\beta=.247$ for

domestic), meaning as student-athletes rated good citizen and self-esteem higher, their institutional attachment scores went up. The relationship with social status, meanwhile, was negative ( $\beta=-.281$ for international, $\beta=-.251$ for domestic), meaning as social status scores went up, institutional attachment scores went down. 
Table 9.

Multiple Regression Results for Purpose of Sport Factors Predicting Institutional Attachment for International Student Athletes

\begin{tabular}{lccc}
\hline Variable & $B$ & $S E B$ & $\beta$ \\
\hline Social Status & -0.293 & 0.085 & $-0.281^{* *}$ \\
Good Citizen & 0.481 & 0.167 & $0.265^{* *}$ \\
Self-Esteem & 0.406 & 0.159 & $0.234^{*}$ \\
Physically Active & -0.159 & 0.101 & -0.134 \\
Mastery/Cooperation & 0.164 & 0.105 & 0.122 \\
Competitiveness & 0.084 & 0.125 & 0.061 \\
High Status & -0.064 & 0.122 & -0.043 \\
\hline
\end{tabular}

Note. $R^{2}=.185$, Adjusted $R^{2}=.151 .{ }^{*} p<.05 .{ }^{* *} p<.01$

Table 10 .

Multiple Regression Results for Purpose of Sport Factors Predicting Institutional Attachment for Domestic Student Athletes

\begin{tabular}{lccc}
\hline Variable & $B$ & $S E B$ & $\beta$ \\
\hline Social Status & -0.273 & 0.122 & $-0.251^{*}$ \\
Self-Esteem & 0.530 & 0.254 & $0.247^{*}$ \\
Competitiveness & 0.228 & 0.179 & 0.147 \\
Mastery/Cooperation & -0.135 & 0.134 & -0.101 \\
Good Citizen & 0.184 & 0.233 & 0.097 \\
High Status & 0.093 & 0.185 & 0.056 \\
Physically Active & -0.068 & 0.157 & -0.053 \\
\hline Note. $R^{2}=.139$, Adjusted $R^{2}=.080 . * 0.05$. & & &
\end{tabular}




\section{Summary of Results}

The results of this study revealed the following findings. Statistically significant differences do exist between international and domestic student-athletes in terms of social adjustment to college and institutional attachment to their college. Domestic studentathletes demonstrated better social adjustment to college and were more attached to their institution than their international counterparts. In regards to how both international and domestic student-athletes view the purpose of collegiate sport, only one of seven factors was statistically significant, namely competitiveness. Domestic student-athletes agreed more strongly that emphasizing competitiveness is an important thing collegiate sport should do compared to international student-athletes.

A series of multiple regression analyses revealed personal views of purpose of sport could not be used to predict how student-athletes, domestic or international, would socially adjust to college. A relationship between view of college sport and institutional attachment does appear to exist, however. Both international and domestic studentathletes demonstrated significant positive correlations between college sport helping selfesteem and institutional attachment, and a negative correlation between college sport elevating social status and institutional attachment. In addition, international studentathletes who felt college sports helped them become good citizens were more attached to their United States college. 
CHAPTER V

\section{DISCUSSION}

Over 10,000 international student-athletes participated in NCAA competition during the 2004-05 school year, a number which has grown rapidly over the past decade (NCAA, 2006b). Relatively little is known, however, about the experiences of these cross-cultural sojourners, although evidence exists to suggest their academic and athletic experiences are different from two other heavily studied groups: (a) international students (non-athletes) and (b) domestic student-athletes. The current study had three purposes: (a) to detect differences in college adjustment between international and domestic studentathletes, (b) to detect differences between perceived purpose of sport between international and domestic student-athletes, and (c) to determine whether perceived purpose of sport predicts ability to socially adjust to college or become more attached to the college for either domestic or international student-athletes. Eleven specific research questions guided the study of these three purposes. The following chapter will address all three purposes by examining data collected to answer each specific research question posed in Chapter I.

Past research suggests national sport policies vary greatly in regards to implementation, emphasis, and opportunity (Chalip, 1995; Green, 2004; Green \& Oakley, 2001; Harvey, Beamish, \& Defrance, 1993; Stahl, Rutten, Nutbeam, \& Kannas, 2002). These differences can lead sport participants to believe certain aspects of sport 
participation are more important than others. In addition, few nations outside the United States implement sport policies in which participation is associated with school attendance (Rubingh \& Broeke, 1998). These national sport policy differences, coupled with the impact of cross-cultural adjustment (Adler, 1985; Church, 1982), offered strong support for an investigation into potential differences in how international studentathletes adjusted to their experience and perceived collegiate sport in their new environment. In addition, a handful of researchers have already noted certain characteristics and affective coping mechanisms of athletes who play sport for extended periods of time in a culture other than their native one, including international studentathletes (Bale 1991; Craven, 1994; Ridinger \& Pastore, 1998). The work of these researchers offered a foundation for the current study.

\section{Adjustment to College}

The first purpose of this study was to determine if international student-athletes adjust to college differently than domestic student-athletes. The first two research questions posited in this study were: (a) "Are there significant differences between international student-athletes and domestic student-athletes in their ability to socially adjust to college?” and (b) “Are there significant differences between international student-athletes and domestic student-athletes in their institutional attachment to their United States college?” A discussion of these two questions, based on the data collected and a review of the literature, follows.

Through years of research, the team of Baker and Siryk (1984; 1986; 1989) suggested college adjustment can be categorized into four different components: (a) academic adjustment, (b) social adjustment, (c) personal-emotional adjustment, and (d) 
institutional-attachment. Previous research has suggested international students do not adjust as well socially to college as domestic students, nor do they have as strong of an institutional attachment (Kaczmarek, Matlock, Merta, Ames, \& Ross, 1994; Ridinger \& Pastore, 1998). Some researchers have linked successful adjustment for international students to social connections made within the new campus environment (Chapdelaine \& Alextich, 2004; Constantine, Anderson, Caldwell, Berkel, \& Utsey, 2005; Westwood \& Barker, 1990). Ridinger (1998) found international student-athletes adjusted better to college than international students who were not athletes. Popp (2006) found international student-athletes also believed they adjusted well socially to college and became attached to their institution. International student-athlete participants in the Popp study attributed their strong adjustment in part to the relationships they formed with other members of their team, an option not readily available for international students who are not part of intercollegiate athletic teams.

\section{Social Adjustment}

Westwood and Barker (1990) suggested social adjustment for international students was better for those students who participated in a peer-partnering group, which created social connections with host nationals. Church (1982) noted the extent and nature to which international sojourners made connections with host nationals often impacted their ability to socially adjust to the new culture. These suggestions, in conjunction with the findings of Ridinger (1998) and Popp (2006), would indicate international studentathletes would likely have higher levels of social adjustment to college than international students, and would post scores similar to those of domestic student-athletes. On the other hand, Rajapaksa and Dundes (2002) found relationships with host nationals may 
not be as strong an indicator of social adjustment as other researchers have suggested for international students.

In the current study, Baker and Siryk’s (1989) Student Adaptation to College Questionnaire (SACQ) was utilized to detect differences between international and domestic student-athletes on a scale of social adjustment to college. The international student-athletes reported significantly lower social adjustment scores than their domestic team mates, although both groups reported above average mean scores. This significant difference challenged an earlier finding by Ridinger (1998), who reported no significant difference between international and domestic student-athletes scores on the social adjustment scale of the SACQ. One likely reason for this discrepancy was the difference in samples for the two studies. The Ridinger (1998) study used a small sample of just 16 international student-athletes, all attending the same institution, while the current study collected data from a national sample. Perhaps the single school in the Ridinger study offered international student-athletes more resources which could have aided in their adjustment. Also, the current study collected data from over 170 international studentathletes, adding a strong element of generalizability to the study compared to the Ridinger paper.

Several reasons exist to help explain why international student-athletes may not socially adjust as well to college as their domestic teammates. Baker and Siryk (1989) state lower social adjustment scores on their questionnaire correlated with, among other things, “less participation in social activities...less success in separating from home ties and establishing social autonomy...greater sense of loneliness...less perceived social support, and perceptions of little opportunity for involvement in social activities” (p. 15) 
at their school. Because international student-athletes travel greater distances to attend college than domestic student-athletes, they may experience more anxiety because of the extended difficulty in maintaining ties to home. Greater cultural distance between the native culture and new culture has been shown to lessen ability to adjust (Adler, 1975; Church, 1982; Rajapaksa \& Dundes, 2002).

International student-athletes certainly have high involvement in one aspect of a university's social offerings--intercollegiate athletes--but may not be as involved in other activities. This may be due to the time commitment involved in NCAA Division I sport participation, a lack of involvement by teammates who may influence international student-athlete social activity decisions, or language barriers. Also, international studentathletes are often unfamiliar with the concept of elite competitive sport being linked to academic achievement. Perhaps the idea of athletic eligibility determined by academic success in college, and vice versa, forces international student-athletes to concentrate more on their academic life than their social one. Also, for those who are non-native English speakers, studying just takes more time than for other students. Like other international students, international student-athletes must overcome language and culture barriers as they work to assimilate into the college setting. Parr, Bradley, and Revathi (1992) found contact with extended family and cultural differences were the two biggest concerns for international students. Those two issues would also affect international student-athletes and their social adjustment, despite their acceptance into a sport team.

As Adler and Adler (1985) point out, student-athletes often have many of their daily decisions made by coaches and athletics administrators who determine what classes student-athletes take, when they will study and train, where they will live, and even what 
and when they will eat. Perhaps this extended involvement by athletics administrators prevents international student-athletes from making their own decisions about becoming involved in other school activities. In addition, Kimball and Freysinger (2002) suggested intercollegiate sport participation may not necessarily be considered by some studentathletes to be a social activity which would help relieve stress, but rather a stressinducing activity which may actually increase tensions and feelings of isolation for minority groups. Some international student-athletes may be the only representative from their native country on the team and the extended time commitment required for Division I athletes does not allow them as much free time to socialize with other international nonathlete populations at their school. The Kimball and Freysinger study examined differences in race and gender, but perhaps national origin could be added to the list.

Ladd and Ruby (1999) suggested one reason international students avoid involvement in other campus activities is not so much because they are nervous or timid, but because campus offerings are not very global in their scope. Ladd and Ruby found the international students in their study were disappointed in campus offerings such as cafeteria menus and intramural sports, which were quite Americanized. Meanwhile, in Parr, Bradley, and Revathi’s (1992) work, the item “socialization” ranked as the second lowest concern for international students, suggesting perhaps social adjustment to college simply might not be a major concern for international student-athletes, and therefore they did not rank it as highly in the survey. Bale (1991) suggested international studentathletes are primarily concerned about obtaining a degree and the athletic experience while enrolled in college. Perhaps making friendships and getting involved in other 
campus activities do not have very high priorities among international student athletes’ own goals in their cross-cultural experience.

\section{Institutional Attachment}

Baker and Siryk (1989) defined institutional attachment as the degree to which a student is committed to educational and institutional goals of his or her college and the degree of attachment or bond to that specific school. Low scores on this scale correlate with the high possibility of leaving school and less overall satisfaction with the school experience. The current study revealed a significant difference in the scores on the institutional attachment scale, with international student-athletes indicating lower institutional attachment than domestic student-athletes.

One explanation for the difference in scores could be international studentathletes’ unfamiliarity with NCAA schools and sanctioned competition. While most domestic student-athletes grow up in an environment where the athletic exploits of universities, particularly Division I institutions, are well documented through media accounts, international student-athletes are not nearly as familiar with such achievements. Domestic student-athletes likely knew about their school and its sports teams before they were even recruited, and in most cases had access to media accounts and conversations with individuals familiar with the school while making their decision about college attendance. In addition, NCAA competition and many of its rules and regulations are well-documented or well-known within United States’ high school athletics circles. On the other hand, because the NCAA does not have as great a presence overseas, many international student-athletes are not as familiar with its organization, structure, and member institutions (Bale, 1991; Popp, 2006). This unfamiliarity may contribute to lower 
institutional attachment because international student-athletes did not grow up cheering for certain teams or valuing the achievements of particular college sport programs.

Another explanation might revolve around expectations of the college experience. International student-athletes elect to accept an athletic scholarship to a United States university for several reasons such as: (a) the appeal of an international experience or adventure; (b) to gain better athletic experience through different coaching, better facilities, or improved competition; (c) the recommendation of friends and coaches; and (d) to receive a college education for free or at a reduced tuition rate (Bale, 1987; 1991; Popp, 2006). For many international student-athletes, most United States colleges offer an opportunity to meet these needs, regardless of school size or location. For domestic student-athletes, many of the motivations for college attendance may be similar, but the reputation and appeal of a specific school likely become more important variables. Domestic student-athletes are probably more likely to choose a school closer to home, with a better athletic reputation, one which their high school friends attend, or in a desirable location, all of which would create a stronger institutional attachment.

In summary, the first portion of the current study did reveal significant findings among both research questions posed. International student-athletes scored significantly lower on both social adjustment and institutional attachment scores as compared to domestic student-athletes. Several reasons exist which may help to explain this finding, but further research is needed to confirm such justifications.

\section{Perceived Purpose of Sport}

People participate in sports for several reasons. In her work with high school student-athletes, Duda (1989) developed a scale to determine the reasons for sport 
participation. Her scale, the Purpose of Sport Questionnaire, includes seven factors or reasons for participation. This scale, which has been used with college athletes (White, 1995), and amateur and professional athletes in Europe (Carpenter \& Yates, 1997;

Treasure, Carpenter, \& Power, 2000), was utilized in the current study to determine if differences existed between international and domestic student-athletes in their motivation for sport participation. The seven original factors from Duda’s scale were accepted for this study and provided the basis for the seven research questions examining perception of purpose of sport. Of those seven factors, only the competitiveness factor revealed a statistically significant difference between scores from international and domestic student-athletes.

\section{Competitiveness}

When participants were asked whether an important thing college sport should do is emphasize competitiveness, domestic student-athlete responses were significantly higher than international student-athletes. This finding echoed Popp’s (2006) research which found international student-athletes felt United States coaches emphasized a "killer instinct" and a "win-at-all-costs" mentality from a young age much more than did youth coaches in other countries. Such an emphasis could affect attitudes of domestic college student-athletes. In addition, domestic student-athletes are more aware of the media attention given to NCAA Division I competition than international student-athletes, which would likely add to a greater emphasis on the competitive nature of collegiate sport.

In addition, international student-athletes might be more likely to emphasize other aspects of the college experience besides just athletics, which may not be the case with 
domestic student-athletes. Past research (Bale, 1987; 1991; Popp, 2006) suggests

international student-athletes have several motivations for attending college in the United States, including a high-emphasis on obtaining a college degree. According to NCAA data (NCAA, 2006a), international student-athletes also had a much higher graduation success rate (GSR) than domestic student-athletes. Perhaps this difference could be attributed to domestic student-athletes' greater emphasis on the competition aspect of college athletics. Past research has suggested student-athletes often define a sense of self based on athletic accomplishment (Adler \& Adler, 1985; Howard-Hamilton \& Sina 2001) and student-athletes have difficulty with dual-role personalities (Killeya-Jones, 2005). If international student-athletes place a higher emphasis on obtaining a degree, perhaps they emphasize athletic success less, which could account for the difference in competition rating.

No significant differences existed between international and domestic studentathletes' responses for the other six purpose of sport factors. Such findings were slightly surprising based on the literature. Suggestions as to why no such differences were found follow.

Mastery and Cooperation

The research question regarding differences in perception of purposes of sport asked whether differences existed between international student-athletes' and domestic student-athletes' views of the purpose of sport as a means of mastery and cooperation of athletic skill. From the data collected, no significant difference for this factor emerged. This particular factor did have the lowest reliability score and was reduced from three 
items to two items after examining the data in order to improve scale reliability, meaning any results should be accepted with caution.

Based on Popp (2006), international student-athletes were expected to rate the mastery and cooperation aspects of collegiate sport higher than domestic student-athletes, although this was not the case in this study. Duda (1989) found a significant link between high mastery and cooperation scores and high task-oriented motivation. Task-oriented motivation stems from self-referenced subjective standards of success. In other words, individuals evaluate success based on their own personal standards for a task, such as competence, perceived ability, and mastery. Ego-orientation opposes task-orientation. If using an ego-orientated barometer, an individual evaluates success through comparison of others. For instance, success might be measured by comparing one's results to others'. Despite the lack of a significant difference for this factor, the current study did reveal an interesting finding. The original mastery and cooperation factor contained three items. If the third item, which also happened to score reasonably low on Duda's (1989) original factor analysis of scale items, was removed, the Cronbach’s alpha scale reliability rating improved from .591 to .626. This improvement also meant only two items remained in the scale. One of those items, the statement "A very important thing college sports should do is teach us to be satisfied when we tried our best,” was rated significantly higher $(p$-value $=.05)$ by international student-athletes as compared to domestic student-athletes. This difference may suggest in some ways international student-athletes are more motivated by task-oriented principles of NCAA sports as compared to their domestic team mates. This finding warrants further investigation, including measurement on a refined scale. 


\section{Physically Active Lifestyle}

Regarding the research question connecting a physically active lifestyle to a purpose of sport, mean scores were quite high, but no significant difference existed between international and domestic student-athletes. The level of athletic competition at the NCAA Division I college level is quite high compared to most other levels of amateur sport found around the globe. Because of this competitive environment and the high athletic demands placed on student-athletes, finding no difference between international and domestic student-athletes in their view that college sports emphasizes a physically active lifestyle was not a surprise. Digel (1995) suggested a tri-level pyramid of sport participation and/or development, with mass-participation sport at the base, competitive sport in the middle, and high-performance sport at the apex. NCAA Division I sport would likely be classified as high-performance sport because participation is very exclusive and individuals and team champions at this level often succeed in international and professional sport. Playing sport to promote a physically active lifestyle could certainly be a motive for people at the mass-participation sport level, but is likely not one of the primary motivations for participants at the high-performance sport level. Because NCAA Division I student-athletes, regardless of origin, chose to play sport at such a high-level, the lack of difference in physically active lifestyle scores makes sense.

\section{Good Citizenship}

In regards to the question of whether a difference exists between how international and domestic student-athletes view college sports as teaching good citizenship, no significant difference was found at the .05 level. This finding was a mild surprise. The factor "good citizenship" incorporated facets such as loyalty, sacrifice, and 
respect of authority. Previous research (Carpenter \& Yates, 1997; Treasure, Carpenter, Power, 2000; White, 1995) found good citizenship, which was re-labeled "sportspersonship" in two of the studies, was rated lower by athletes who were located higher (high performance level) on the Digel (1995) sport-development pyramid. In two of the studies, amateur athletes rated good citizenship higher than professional or semiprofessional athletes, while in the other study, intramural college athletes rated good citizenship higher than varsity college athletes. While both groups in the current study were playing elite sport at the NCAA Division I level, previous research (Bale, 1991; Popp, 2006) has illustrated the move from elite club competition outside the United States to NCAA college competition for international student-athletes is a more parallel move than the jump from high school to college athletics for domestic student-athletes. Because of this, international student-athletes typically have spent a longer period of time in the upper level of the Digel pyramid, which could have affected good citizenship scores. However, such a hypothesis was not supported in the current study.

\section{High Status}

Regarding the question of whether a difference existed between international and domestic student-athletes in terms of college sport granting them higher status, no significant difference was revealed. In fact, mean scores between the two groups were practically identical (4.20 for domestic, 4.21 for international). This finding was surprising. The high status factor on the scale consisted of items such as: (a) giving us a chance to obtain a college education, (b) the chance to move into a good job, and (c) earn more money. Playing NCAA Division I sports does seem to open doors for domestic student-athletes who seek jobs in the United States because of the high-profile status of 
Division I sports and the belief athletes make good employees (Argent \& Robinson, 2005). However, employers outside the United States may not be as aware of the nature of NCAA Division I sport participation, including its high demands of time and commitment. Yet international student-athletes felt their participation in NCAA Division I sport could indeed by parlayed into better jobs at the same rate as domestic studentathletes. Perhaps a large percentage of international student-athletes hope to remain in the United States after completing college. This is an interesting finding which deserves further investigation.

Self-esteem

Regarding the question of whether a difference existed between international and domestic student-athletes in terms of college sport boosting self-esteem, no significant difference was revealed. In fact, again, mean scores between the two groups were practically identical (4.35 for domestic, 4.34 for international). This finding was not surprising. In past studies examining other groups of athletes, no significant differences in sport developing self-esteem were detected between male and female high school athletes (Duda, 1989) and between intramural and varsity college athletes (White, 1995). In the current study, college sports aiding in self-esteem was the highest rated factor of all seven presented. The results indicate college student-athletes, regardless of nationality, find college sports as a boost to self-esteem. Nearly all student-athletes competing in NCAA Division I sports were recruited and many of them were offered at least some form of athletic scholarship. Such actions would likely increase confidence levels and feelings of importance linked to their athletic ability, which are measures of self-esteem in the purpose of sport questionnaire. 


\section{High Social Status}

In regards to the question of whether a difference exists between how international and domestic student-athletes view college sports as enhancing social status, no significant difference was found. This finding was slightly surprising. Because NCAA Division I sports enjoy a higher profile in the United States, due to media coverage as well as school marketing initiatives, it would have been understandable for domestic student-athletes to rate this factor higher than international student-athletes. International student-athletes playing collegiate sport in the United States do not exhibit their skills in front of peers from their native county. International student-athletes certainly make friends while in college, but moving up the social ladder would seem an unlikely motivation for such a cross-cultural sojourn. On the other hand, elevated social status would seem to be a natural motivation for many domestic student-athletes to play college sport.

White's (1995) work, however, would support the findings of this study. In her research, she found no difference in the social status dimension between varsity athletes and students who played intramural sport. In White’s (1995) study, Duda’s (1989) study, and the current study, social status had the lowest mean score of any purpose of sport factor for all participant groups. In all three studies, elevated social status mean scores were very close to 2.5 on a 5-point Likert-type agreement scale, indicating participants generally felt neutral regarding this particular aspect and its role in sport participation. Taken together, the results of these three studies seem to indicate student-athletes do not necessarily consider enhanced or elevated social status as a primary motive to play sport. 


\section{Summary}

In summary, the second portion of this study, which examined differences between international and domestic student-athletes in their perception of college sport, found only one significant difference among seven factors. This difference occurred in the factor of competitiveness, with international student-athletes rating the purpose of sport as being about competition lower than domestic student-athletes. Among the other six factors, several were expected to produce significant differences, yet did not. Explanations were offered regarding the findings.

Predictive Ability of Purpose of Sport for Adjustment to College

A third goal of this study was to determine whether student-athletes' perception of the purpose of sport could predict what sort of social adjustment to college they would make and whether it impacted their institutional attachment to the school. Positive selfidentity or self-concept has been closely linked to athletic achievement for many college student-athletes (Howard-Hamilton \& Sina, 2001). In addition, the way in which studentathletes balance athletic roles and academic roles has been shown to impact adjustment to college (Killeya-Jones, 2005). Because of these factors, it would seem student-athletes’ motivations for playing sport, and their view of the purpose of sport, could certainly be tied to their ability to adjust. This current study conducted several exploratory regression analyses to determine whether this hypothesis was indeed true. The results of this portion of the study were mixed.

Purpose of Sport Factors and Social Adjustment

Simultaneous entry regression analyses revealed purpose of sport factors provided no significant prediction of social adjustment for either international or domestic student- 
athletes. This finding was surprising. The literature is mixed on the connection between athletic accomplishment and social development. While Howard-Hamilton and Sina (2001) found student-athletes developed positive senses of industry and identity in relation to athletic achievements, they did state student-athletes' focus on this connection could overshadow social development in other areas during college years. Division I student-athletes have been shown to give themselves higher assessments of overall wellbeing compared to non-student-athletes (Aries, McCarthy, Salovey, \& Banaji, 2004) and have been shown to possess higher levels of perceived social acceptance (Curry \& Rehm, 1997). Athletic participation has also been shown to relate positively to student-athletes' personal and social well-being (Cantor \& Prentice, 1996). Thus a connection seems to exist between college athletic participation and perceived social competence. However, Bostic (1979) found student-athletes indicated more social problems adjusting to social life in college than non-athletes and Adler and Adler (1985) pointed out the studentathletes in their study suffered social isolation.

The results of the current study indicated no relationship between how a studentathlete, regardless of origin, views the purpose of participating in college athletics and social adjustment to college. Both domestic and international student-athletes did rank their social adjustment to college as above average, with mean scores for both groups higher than the scores collected from the Ridinger (1998) study on the same social adjustment scale. And, in fact, Baker and Siryk (1989) advise that it is not unusual for SACQ scores to rank lower than typical achievement test scores, meaning average results (mid-point of the scale) do not indicate poor adjustment, but rather satisfactory adjustment. Thus, the high social adjustment scores in this study are even more 
impressive. However, based on the results of this study, other factors outside purpose of sport are at play in those social adjustment scores, further clouding the concept of a link between athletic participation and social adjustment to college for Division I studentathletes. This issue needs further examination as to why Division I student-athletes exhibit high social adjustment ability and will likely continue receiving scholarly attention.

A lack of a relationship between the factor "social status" as a purpose of sport and social adjustment to college seemed particularly perplexing in this study. This lack of relationship would seem to indicate student-athletes at the college level truly are unmotivated by the enhanced social status aspect of playing Division I sports. If studentathletes were motivated by their status as athletes in helping to make friendships and boost prestige among peers, a relationship would have likely emerged. Instead it did not, perhaps indicating student-athletes think being an athlete does not actually enhance their social status. Or perhaps an enhanced sense of social status stemming from being an athlete is not important to them. A third explanation could be participants in the study did not want to appear egotistical and ranked college sports enhancing social status as a low factor on the survey even though they did enjoy certain social benefits of being a studentathlete. While the lack of any purpose of sport factor providing a relationship to social adjustment seemed surprising, this particular purpose of sport factor relationship with social adjustment may be the one which begs the most for further exploration. Purpose of Sport Factors and Institutional Attachment

Simultaneous entry regression analyses revealed purpose of sport factors provided several significant predictions of institutional attachment for both international and 
domestic student-athletes. Among international student-athletes, three of the seven purposes of sport factors predicted close to $19 \%$ of the variance in institutional attachment, which was a significant finding. International student-athletes who rated good citizenship and self-esteem high, while rating social status low, had higher levels of institutional attachment. While the current study was exploratory in nature, these findings were not surprising. Of all factors, good citizenship accounted for the second highest prediction percentage of institutional attachment in the regression equation for international student-athletes. The good citizenship factor included things such as college sports teaching loyalty, requiring sacrifice, and emphasizing respect of authority. If international student-athletes believed college sports emphasized these things and they were indeed members of a college sport team, logic would suggest they would be more loyal, more willing to sacrifice for the betterment of the team, and would respect the feelings and opinions of coaches and athletic administrators. Thus, the connection between good citizenship and institutional attachment is not far-fetched as international student-athletes likely would associate their athletic program with their institution.

What is interesting, however, is domestic student-athletes rated good citizenship at a very similar level to internationals, but those ratings did not correlate with domestic student-athlete institutional attachment. Only self-esteem (positive relationship) and social status (negative relationship) significantly predicted domestic student-athletes’ institutional attachment. In fact, those two factors predicted $14 \%$ of the variance in the regression equation for institutional attachment among domestic student-athletes.

Perhaps domestic student-athletes professed to sport teaching good citizenship characteristics, but did not really internalize those characteristics. In other words, maybe 
domestic student-athletes are anxious to say college sports teach things such as loyalty and sacrifice for the betterment of the team, but they are not as ready to internalize those concepts when it comes to school loyalty, especially when compared to international student-athletes. Domestic student-athletes did actually have significantly higher levels of institutional attachment compared to their international teammates. This high level of attachment, though, seems unconnected to certain aspects related to being part of a sports team as gauged by the good citizenship sport factor. This relationship, or lack there of, seems peculiar and certainly warrants further investigation.

The idea of college sports promoting positive self-esteem, which in turn predicted institutional attachment, seems straightforward, regardless of a student-athlete's national origin. Those who thought college athletics enhance self-esteem were more likely to be attached to their school. If one feels good about one's self, satisfaction with one's environment would seem to follow. High self-esteem as a result of athletic participation would seem to link individuals to their athletics program, and in turn, their college. This correlation was true for both international and domestic student-athletes.

Student-athletes who rated social status low rated institutional attachment high. In essence, these student-athletes were more closely aligned with the school or program, if they were less likely to think college sports was about boosting one's own ego. This seemed to indicate a sense of team unity and program loyalty. If student-athletes were competing in college athletics for personal social gain, they were probably less likely to find satisfaction in the competitive NCAA Division I college environment, where team is often put above individual accomplishment. Individuals who did rate social status high had a lower institutional attachment. If the college athletic experience was about 
elevating social status, a somewhat selfish or ego-driven motive, individuals were not as happy with their school choice or did not feel as committed to their particular institution.

\section{Summary}

In summary, purpose of sport factor scores did not predict any social adjustment ability for either international or domestic student-athletes. However, self-esteem and social status did impact institutional attachment for both groups. The relationship was positive for self-esteem and negative for social status. In addition, the factor good citizenship predicted the highest percentage of institutional attachment for international student-athletes but was not a significant predictor for domestic student-athletes. Possible explanations for these findings were addressed.

\section{Implications}

The current study has several implications for college coaches, athletics administrators, and student-athletes, both international and domestic. While over 10,000 international student-athletes are currently competing at NCAA schools (NCAA, 2006b), only a handful of researchers have sought to understand the college experience for these cross-cultural sojourners, and whether the experience is similar or different to studentathletes from the United States. Understanding the experience of international studentathletes can translate into more effective recruitment of strong international competitors, better retention rates of student-athletes, and more positive experiences for the studentathletes themselves. Findings may be particularly relevant for coaches and administrators working with teams containing a high concentration of international student-athletes, such as tennis or soccer programs. 


\section{Social Adjustment and Institutional Attachment}

Significant differences existed between international and domestic studentathletes in terms of social adjustment to college and institutional attachment. While both groups expressed relatively high levels of social adjustment, domestic student-athletes had an easier time adjusting, which ran contrary to earlier findings by Ridinger (1998). Poor social adjustment to college can lead to a host of other difficulties for college students (Baker \& Siryk, 1989), meaning coaches and athletics administrators may need to look at developing programming within the team or athletic department setting to assist with social adjustment, particularly for schools and teams with a high concentration of international student-athletes. Adler and Adler (1985) suggested student-athletes at the Division I level have many decisions regarding their college experience made for them by coaches and administrators. When those decisions, such as which classes and majors in which to enroll, where to live, and when to study, are not made by the student-athletes themselves, it can stunt the social and intellectual development gained by most nonathlete college students during their years on campus (Howard-Hamilton \& Sina, 2001). Westwood and Barker (1990) found international students who were partnered with a host national had an easier time adjusting to their new culture. Perhaps coaches and athletics administrators could develop a formal partnering program for international student-athletes in which a current domestic student-athlete became a mentor.

In addition, because of the demands placed on Division I college student-athletes, little time or opportunity exists for both domestic and international student-athletes to become involved in other campus activities, which can hamper both social adjustment and institutional attachment. Coaches and administrators need to make a concerted effort 
to allow student-athletes to soak up the full range of experiences college life offers. This need is particularly important for international student-athletes, who are often in the United States for more than just an athletic experience (Bale, 1987; 1991; Popp, 2006).

According to Baker and Siryk (1989) students who score low on social adjustment and institutional attachment scales are more likely to struggle being away from home and are more likely to leave school before graduation. Keeping international student-athlete retention rates high are a concern for coaches and administrators for several reasons. High retention can help lead to a better academic progress rate (APR) score and can assist in developing international recruiting pipelines. Many NCAA Division I schools cannot compete in recruiting battles for elite domestic talent, but those schools can use international connections and recruitment to help level the competitive playing field.

Several researchers (Adler, 1975; Befus, 1988; Church, 1982; Craven, 1994) examined the U-curve concept of cross-cultural adjustment and the need for pre-sojourn preparation to minimize the effects of culture shock. International students have demonstrated lower levels of social adjustment to college (Barker \& Westwood, 1990; Kaczmarek, Matlock, Merta, Ames, \& Ross, 1994, Ridinger, 1998), and now this current research has demonstrated a similar phenomenon involving international student-athletes. Coaches, school administrators, and NCAA officials can help limit the impact of culture shock for international student-athletes by better preparing recruits for their journey to the United States before they ever leave their home nation. Recruiting information should include more than athletic materials. Recruiting pieces such as information regarding the college experience in the United States, student visa information, and facts and figures regarding other international students' experiences at college could be welcome additions 
for international student-athlete prospects. NCAA administrators may want to consider revising regulations limiting the type of recruiting materials departments are allowed to send to prospective athletes when those materials are sent outside the United States. In addition, the NCAA should establish better means of communicating eligibility requirements to prospective student-athletes in other countries and become more lenient in their decisions regarding amateur status of international student-athletes. Perhaps the NCAA could train ambassadors or counselors in other countries to help assist prospective student-athletes in their transition to becoming eligible student-athletes.

\section{Purpose of Sport Factors}

Regarding the purpose of sport factors, competitiveness was the only factor in which a significant difference existed between domestic and international studentathletes. What this could tell college coaches and recruiters is perhaps they should slightly alter recruiting pitches made to international student-athletes. An international student-athlete unfamiliar with NCAA conferences will likely not be motivated by the chance to win a Big 10, Atlantic Coast Conference, or Big East title, yet such pitches are commonplace during the recruitment of domestic student-athletes.

Closer examination of the purpose of sport scores could also give coaches reasons to alter recruiting methods for international student-athletes. Mastery and cooperation was the second highest rated purpose of sport factor among international student-athletes, but was fifth out of seven factors for domestic student-athletes. The mastery and cooperation factor has been linked to task-oriented motivation (Duda, 1989) meaning perhaps international student-athletes are more motivated by the ideas of selfimprovement and the intrinsic rewards of collegiate competition than are domestic 
student-athletes. If this is the case, college coaches and recruiters would be better off emphasizing the skill development aspects of college sport as opposed to the competitive rewards.

Another interesting finding with the current study was student-athletes, regardless of national origin, rated college sport as enhancing career status relatively high. International and domestic student-athletes rated the factor nearly identical and as the third highest rated factor among the seven listed. This is interesting for two reasons. First, in White's (1995) study, Division I student-athletes rated the career status factor fairly low; fifth among the seven factors. Second, international student-athletes rated this factor as high as domestic student-athletes, yet it is doubtful many of their potential employers in their native nations will be familiar with NCAA competition or the schools they attended while studying in the United States. It is hard to draw conclusions from this single item, but further examination could reveal intriguing findings. Do employers favor college graduates who play collegiate sport, even if the employer knows little about the type or level of competition? Or do most international student-athletes intend to remain in the United States after they graduate and feel their status as a student-athlete will open doors to potential employers?

\section{Purpose of Sport Predicting Adjustment to College}

As discussed earlier, it was surprising in this study to not find any purpose of sport factor significantly predicting social adjustment. This in itself is telling to researchers and college athletics administrators who strive to ensure student-athletes are able to develop both athletically and socially during their critical college years. Being a student-athlete has been shown to impact social experience in college (Adler \& Adler, 
1985; Howard-Hamilton \& Sina, 2001). Based on this study, however, the motivation or purpose of participating in college sport does not seem related to a student-athlete's ability to fit in socially in college. Perhaps student-athletes are simply becoming better at adjusting to college life outside of athletics and some of the measures employed at schools, such as the NCAA's CHAMPS/Life Skills program, are effective. Or perhaps other reasons exist for successful social adjustment of student-athletes. Finding predictors of social adjustment will help student-athletes get more out of their college experience beyond just athletic accomplishment, but more work is necessary to uncover this predictors.

Student-athletes who felt college athletics should enhance self-esteem were more attached to their institution. This finding is certainly not earth-shattering, but when taken in conjunction with other studies on student-athlete well-being (Adler \& Adler, 1985; Howard-Hamilton \& Sina, 2001; Killeya-Jones 2005), it reinforces the idea that for student-athletes, athletic experience is closely linked with college satisfaction. The influence and guidance coaches and administrators exert over student-athletes does not stop on the practice field or playing court. These individuals wield a great deal of influence over the student-athlete's college experience and can leave a powerful impact. In addition, college fund-raisers may be interested to see this link between self-esteem and institutional attachment. Student-athletes who have their self-esteem boosted by playing college sports may be more likely to donate to athletic programs after graduation because of their strong institutional attachment. This potential tendency becomes more intriguing when considering international student-athletes. As the numbers of 
international student-athletes continue to rise, will they become active alumni who contribute to athletic programs after graduation?

The link between social status and institutional attachment provides an interesting implication for student-athletes themselves, regardless of national origin. Student-athletes who rated college sport as enhancing social status highly were not as likely to have a tight connection with their school. This finding would seem to imply student-athletes who expect the college athletic experience to help them be popular among their peers and make many friends, might not have been motivated by the right reasons when they decided which college they will attend. Those student-athletes who indicated low social status on the purpose of sport scale were significantly more satisfied with their institution.

International student-athletes also rated good citizenship as a significant predictor of institutional attachment. The items comprising good citizenship included things like willingness to sacrifice for the team, loyalty, and respecting authority, which provide a logical transition to institutional attachment. However, good citizenship did not predict institutional attachment for domestic student-athletes. For coaches and college administrators, recruiting international student-athletes who value good citizenship may result in better retention rates and team members who are more dedicated to their athletics program. This finding also raises a red flag regarding the motivation of domestic student-athletes who think college athletics are about teaching good citizenship, but whose ratings of institutional attachment do not correspond.

\section{Summary}

To summarize, this study has implications for coaches, athletics administrators, NCAA officials, and student-athletes. The four or five years student-athletes spend at a 
university can have a great impact on their social well-being. In addition, because so many international student-athletes are now coming to the United States, it is important to know what attracts them to NCAA schools and what impacts their stay in the United States. Such factors can lead to more successful recruitment of international studentathletes and could even have repercussions for the athletic programs after international student-athletes graduate.

\section{Future Research}

The dearth of research regarding international student-athletes opens many doors for future research on the topic. The current study focused on differences between domestic and international student-athletes. Categorizing all international student-athletes in one group, however, can be problematic, particularly considering the differences in national sport policy from one country to another. Future research should examine differences among different sub-groups of international student-athletes such as country, region, or continent of origin. National differences may be hard to detect through quantitative means because it is difficult to obtain adequate sample sizes from all desired countries. For example, this study contained a national sample of 174 international student-athletes, but 49 different countries were represented, an average of 3.6 studentathletes per country. Thus, future studies should either focus on region or utilize qualitative means to account for the difficulty in obtaining an adequate sample size.

Other demographic details could also shed light on future studies regarding international student-athletes. Key variables include: (a) gender, (b) sport played (team/individual or revenue/non-revenue), (c) number of years spent in the United States prior to college, (d) prior international travel experience, (e) academic success, (f) level 
of English proficiency, and (g) international population in the community, on campus, or within the athletic department. Differences could be detected based on individual variables or linear combinations of variables.

Certain findings from the current study could also be expanded upon in future work. Future researchers should examine the reasons behind why international studentathletes rated the competition aspect of college sports lower than domestic studentathletes. They should also look at why international and domestic student-athletes rated college sports as enhancing career status at identical rates. Finally, the link between athletic accomplishment and social adjustment needs to be better understood. Exploratory qualitative research may be the best option for shedding light on these issues.

Another important area which needs to be addressed is pre- and post-sojourn assessment for international student-athletes. What happens when these international students return to their home countries? What are their attitudes toward their United States experience? What percentage stays in the United States after graduation? Research regarding this population has mainly focused on experiences while enrolled in school (Popp, 2006; Ridinger 1998; Ridinger \& Pastore, 2000a; Stidwill, 1984). Bale (1987; 1991) remains the only researcher to collect data on international student-athletes after their experience has ended. Much of his work was confined to Western Europe and was gathered nearly 20 years ago, prior to a massive increase in international student-athlete recruitment.

Future studies should examine international student-athlete recruits' knowledge of NCAA rules, including eligibility status, before they traveled to the United States. Also, experiences of international student-athletes after they have completed their athletic 
eligibility could form the basis of intriguing and beneficial studies. Such research could help answer questions as to: (a) whether international student-athletes were satisfied with their international experience, (b) whether they were satisfied with their educational experience, (c) how the experience affected their effort to land a job, (d) whether they remain in contact with their school, (e) if they assist others from their homeland in becoming international student-athletes, (f) how the experience impacted their athletic career, (g) whether their age when entering college in the United States made a difference in their adjustment, and (h) how their role on the team as either a star or minor contributor impacted their experience.

\section{Summary of Study}

Three relationships were examined in this study. The first compared international and domestic student-athletes in their adjustment to college. Results showed domestic student-athletes socially adjusted to college better and were more attached to their institution than international student-athletes. Both differences were significant. In the second portion of the study, international and domestic student-athletes were again compared, this time in terms of their perception of the purpose of sport. Purpose of sport was measured through seven factors. A significant difference between the two groups existed in only one of those seven factors, namely competitiveness.

The third part of the study examined whether student-athlete's views of the purpose of sport could predict either their social adjustment to college or their level of institutional attachment. Purpose of sport had no significant relationship with either domestic or international student-athletes in regards to their social adjustment to college. Three different items, good citizenship, self-esteem, and social status, all had a significant 
relationship with institutional attachment for international student-athletes. The relationship was positive for both good citizenship and self-esteem, while the relationship was negative for social status. Among domestic student-athletes, self-esteem and social status provided a significant ability to predict institutional attachment. This relationship was positive for self-esteem and negative for social status.

The findings of this study show differences do exist between international and domestic student-athletes in several dimensions of college adjustment and perspectives of the purpose of sport. These differences highlight the need for NCAA coaches and college administrators to be aware of how international student-athletes are adapting to the college environment. They also show international student-athletes have some unique needs and expectations for the college athletic experience as compared to domestic student-athletes. Finally, the results of this study reinforce the idea competitive sport can be approached in many different ways by individual participants, with many variables affecting their perspectives and experiences. 


\section{REFERENCES}

Adler, P. (1975). The transitional experience: An alternative view of culture shock. The Journal of Humanistic Psychology, 15, 13-23.

Adler, P., \& Adler, P. A. (1985). From idealism to pragmatic detachment: The academic performance of college athletes. Sociology of Education, 58, 241-250.

Adler, P., \& Adler, P. A. (1991). Backboards \& blackboards: College athletes and role engulfment, New York: Columbia University Press.

Aiken, L.R. (2000). Psychological testing and assessment. Boston: Allyn and Bacon.

Archer, J., Ireland, J., Amos, S. L., Broad, H., \& Currid, L. (1998). Derivation of a homesickness scale. British Journal of Psychology, 89(2), 205-217.

Argent, E., \& Robinson, L. (2005, June). From the locker room to the boardroom: Developing leaders through sport. Paper presented at the meeting of the North American Society for Sport Management, Regina, Saskatchewan, Canada.

Aries, E., McCarthy, D., Salovey, P., and Banaji, M. R. (2004). A comparison of athletes and non-athletes at highly selective colleges: Academic performance and personal development. Research in Higher Education, 45, 577-602.

Astin, A.W. (1984). Student involvement: A developmental theory for higher education. Journal of College Student Personnel, 25, 297-308.

Astin, A. W. (1993). What Matters in College? Four Critical Years Revisited. San Francisco: Jossey-Bass.

Babiker, I. E., Cox, J. L., \& Miller, P. M. (1980). The measurement of cultural distance and its relationship to medical consultations, symptomatolgy and examination performance of overseas students at Edinburgh University. Social Psychiatry, 15, 109-116.

Baker, R. W., \& Siryk, B. (1984). Measuring adjustment to college. Journal of Counseling Psychology, 31, 179-189. 
Baker, R. W., \& Siryk, B. (1986). Exploratory intervention with a scale mesauring adjustment to college. Journal of Counseling Psychology, 33, 31-38.

Baker, R. W., \& Siryk, B. (1989). Student adaptation to college questionnaire manual. Los Angeles: Western Psychological Services.

Bale, J. (1987). Alien student-athletes in American higher education: Locational decision making and sojourn abroad. Physical Education Review, 10(2), 81-93.

Bale, J. (1991). The brawn drain: Foreign student athletes in American universities, Urbana, IL: University of Illinois Press.

Befus, C. P. (1988). A multilevel treatment approach for culture shock expereicned by sojourners. International Journal of Intercultural Relations, 12, 381-400.

Borrow, H. (1949). Manual for the college inventory of academic adjustment. Palo Alto, CA: Stanford University Press and Consulting Psychologists Press.

Bostic, D. (1979). A comparison of the problems of Black and White college athletes. Unpublished manuscript, University of Florida, 1979.

Brodman, K., Erdmann, A. J., Lorge, I., Gerhenson, C. P., \& Wolff, H. G. (1952). The Cornell medical index health questionnaire: The evaluation of emotional disturbances. Journal of Clinical Psychology, 8, 119.

Brown, T. (2004, December 6). Foreign matter: Influx of internationals in college swimming tugs on bond between campus and country. NCAA News, p 5.

Brown, L., \& Moore, E., (1970). The intra-urban migration process: A perspective. Geografiska Annaler, 52B, 1-13.

Butcher, J., Linder, K., \& Johns, D. (2002). Withdrawl from competitive youth sport: A retrospective ten-year study. Journal of Sport Behavior, 25(2), 145-163.

Byers, W., \& Goossens, L. (2002). Concurrent and predictive validity of the Student Adaptation to College Questionnaire in a sample of European freshman students. Educational and Psychological Measurement, 62, 527-538.

Caldwell, B. (1987). Unpublished data from the University of California Davis.

Cantor, N. E., and Prentice, D. A. (1996). The life of the modern-day student-athlete: Opportunities won and lost. Paper presented at the Princeton Conference on Higher Education, Princeton University, Princeton, NJ. 
Carpenter, P. J., \& Yates, B. (1997). Relationship between achievement goals and the perceived purposes of soccer for semiprofessional and amateur players. Journal of Sport \& Exercise Psychology, 19, 302-311.

Castro-Abad, C. (1995). A human development workshop on cultural identity for international students. Princeton, NJ: Princeton University, Mid-Career Fellowships Program.

Chapdelaine, R. F., \& Alextich, L. R. (2004). Social skills difficulty: Model of culture shock for international graduate students. Journal of College Student Development, 45, 167-184

Chalip, L. (1995). Policy analysis in sport management. Journal of Sport Management, 9, $1-13$.

Chalip, L., Johnson, A., \& Stachure, L. (1996). National sports policies. Westport, CT: Greenwood Press.

Chambliss, D. F. (1989). The mundanity of excellence: An ethnographic report on stratification and Olympic swimmers. Sociological Theory, 7, 70-86.

Church, A. T. (1982). Sojourner adjustment. Psychological Bulletin, 91, 540-572.

Collins, M., \& Buller, J.R. (2000). Bridging the post-school institutional gap in sport: Evaluating champion coaching in Nottinghamshire. Managing Leisure, 5, 200221.

Collins, M., \& Buller, J.R. (2003). Social exclusion from high-performance sport: Are all talented young sports people being given equal opportunity of reaching the Olympic podium? Journal of Sport and Social Issues, 27(4), 420-442.

Comrey, A. L., \& Lee, H. B. (1992). A first course in factor analysis. Hillsdale, NJ: Lawrence Erlbaum Associates, Publishers.

Constantine, M. G., Anderson, G. M., Caldwell, L. D., Berkel, L. A., \& Utsey, S. O. (2005). Examining the cultureal adjustment experiences of African international college students: A qualitative analysis. Journal of Counseling Psychology, 52, 57-66.

Cote, J. \& Haye, J. (2002). Children's involvement in sport: a developmental perspective, in: J.M. Silva \& D. Stevens (Eds) Psychological Foundations of Sport (Boston, Merrill).

Craven, J. (1994). Cross-cultural impacts of effectiveness in sport. In R.C. Wilcox (Ed.) Sport in the global village, (pp. 433-448). Morgantown, WV: Fitness Information Technology, Inc. 
Crano, S. L., \& Crano, W. D. (1993). A measure of adjustment strain in international students. Journal of Cross-Cultural Psychology, 24, 267-283.

Cunningham, J. (2001). Elite sports funding review (Report of the Review Group, Chaired by the Rt Hon Dr Jack Cunningham MP), Department for Culture, Media and Sport: London.

De Boeck, P., \& Rosenberg, S. (1988). Hierarchical classes: Model and data analysis. Psychometricka, 53, 361-381.

Department for Culture, Media and Sport (2000). A sporting future for all, Department for Culture Media and Sport: London.

Digel, H. (1995). Sport in a changing society. Schorndorf, Germany: Verlag Karl Hoffman.

Dillman, D. (2000). Mail and internet surveys: The tailored design method. New York: John Wiley \& Sons, Inc.

Duda, J. L. (1989). Relationship between task and ego orientation and the perceived purpose of sport among high school athletes. Journal of Sport \& Exercise Psychology, 11, 318-335.

Duda, J. L., \& Allison, M. T. (1990) Cross-cultural analysis in exercise and sport psychology: A void in the field. Journal of Sport \& Exercise Psychology, 12, 114131.

Duda, J. L., \& Nicholls, J. G. (1992). Dimensions of achievement motivation in schoolwork and sport. Journal of Educational Psychology, 84, 290-299.

Duda, J., \& Whitehead, J. (1998). Measurement of goal perspectives in the physical domain, in: J. Duda (ed.) Advances in Sport and Exercise Psychology Measurement, pp. 21-48 (Morgantown, WV, Fitness Information Technology).

Elling, A., De Knop, P., \& Knoppers, A. (2001). The social integrative meaning of sport: A critical and comparative analysis of policy and practice in the Netherlands. Sociology of Sport Journal, 18, 414-434.

Erikson, E. (1980) Identity and the life cycle. New York: Norton.

Fox, J. (1991). Regression diagnostics. Thousand Oaks, CA: Sage Publications.

Furnham, A. \& Bochner, S. (1982). Social difficulty in a foreign culture: An empirical analysis of culture shock. In S. Bochner (Ed.), Cultures in contact: Studies in cross-cultural interaction. (pp. 161-198). New York: Pergamon Press. 
Furnham, A., \& Bochner, S. (1986). Culture shock: Psychological reactions to unfamiliar environments. London: Methuen.

Galtung, I. E. (1965). The impact of study abroad: A three-by-three nation study of crosscultural contact. Journal of Peace Research, 2, 258-276.

Gerdes, H. (1986). Freshman adjustment to college: Expectations, satisfaction, and persistence. Unpublished master's thesis, University of Oregon, Eugene.

Giddens, A. (1984). The Constitution of society. Cambridge: Polity.

Godfrey, J., \& Holtham, G. (1999). Sporting lives: A vision for sport in the UK. London: Institute for Public Policy Research.

Goudas, M., Biddle, S,. \& Fox, K. (1994). Achievement goal orientations and intrinsic motivation in physical fitness testing with children, Pediatric Exercise Science, 6, pp. 159-167.

Green, B.C. (2005). Building sport programs to optimize athlete recruitment, retention, and transition: Toward a normative theory of sport development. Journal of Sport Management, 19, 223-253.

Green, M. (2004). Power, policy, and political priorities: Elite sport development in Canada and the United Kingdom, Sociology of Sport Journal, 21, 376-396.

Green, M., \& Oakley, B. (2001). Elite sport development systems and playing to win: uniformity and diversity in international approaches. Leisure Studies, 20, 247267.

Green, S. B. (1991). How many subjects does it take to do a regression analysis? Multivariate Behavioral Research, 26, 449-510.

Harris, B. C. (1988). How the structure and function of social networks relate to loneliness and college adaptation. Unpublished master's thesis, George Washington University, Washington, DC.

Hannum, J. W., \& Dvorak, D. M. (2004). Effects of family conflict, divorce, and attachment patterns on the psychological distress and social adjustment of college freshmen. Journal of College Student Development, 45, 27-42.

Hartmann-Tews, I. (1999). The idea of sport for all and the development or organised sport in Germany and Great Britain. Journal of European Area Studies, 7(2), 145156. 
Harvey, J., Beamish, R., \& Defrance, J. (1993). Physical exercise policy and the welfare state: A framework for comparative analysis. International Review for Sociology of Sport, 28(1), 53-63.

Henry, I., \& Nassis, P. (1999). Political clientelism and sports policy in Greece. International Review for the Sociology of Sport, 34(1), 43-58.

Hill, C. E., Thompson, B. J., \& Williams, E. N. (1997). A guide to conducting consensual qualitative research. The Counseling Psychologist, 25, 517-572.

Horch, H. (1994). Does government financing have a detrimental effect on the autonomy of voluntary associations? Evidence from German sports clubs. International Review for Sociology of Sport. 29, 269-282.

Houlihan, B., \& White, A. (2002). The politics of sport development: Development of sport or development through sport? London: Routledge Taylor \& Francis Group.

Howard-Hamilton, M. F., \& Sina, J. A. (2001). How college affects student athletes. New Directions for Student Services, 93, 35-45.

Kaczmarek, P. G., Matlock, G., Merta, R., Ames, M. H., \& Ross, M. (1994). An assessment of international college student adjustment. International Journal for the Advancement of Counselling, 17, 241-247.

Kealey, D. J. (1978). Adaptation to new environments. Paper presented at the Canadian International Development Agency, Ottawa.

Killeya-Jones, L. A. (2005). Identity structure, role discrepancy and psychological adjustment in male college student-athletes. Journal of Sport Behavior, 28, 167185.

Kimball, A. \& Freysinger, V. J. (2002). Leisure, stress, and coping: The sport participation of collegiate student-athletes. Leisure Sciences, 25, 115-141.

Kirk, D. \& MacPhail, A. (2003). Social positioning and the construction of a youth sports club. International Review for the Sociology of Sport, 38(1), 23-44.

Koski, P. (1995). Organizational effectiveness of Finnish sports clubs. Journal of Sport Management, 9, 85-95.

Ladd, P. D., \& Ruby. R. (1999). Learning style and adjustment issues of international students. Journal of Education for Business, 74, 363-367.

Leong, F. T. L., \& Bonz, M. H. (1997). Coping styles as predictors of college adjustment among freshman. Counselling Psychology Quarterly, 10, 211-221. 
Litsky, F. (2003, June 16). Foreign athlete presence is felt at NCAA meet. New York Times, D8.

Luzzo, D. A., Henao, C., \& Wilson, M. (1996). An innovative approach to assessing the academic and social needs of international students. Journal of College Student Development, 37, 351-352.

Lysgaard, S. (1955). Adjustment in a foreign society: Norwegian Fullbright grantees visiting the United States. International Social Science Bulletin, 7, 45-51.

MacPhail, A., Gorely, T., \& Kirk, D. (2003). Young people's socialization into sport: A case study of an athletics club. Sport, Education, and Society, 8(2), 251-267.

Marida, K. V. (1971). The effect of nonnormality on some multivariate tests and robustness to nonnormality in the linear model. Biometrika, 58(1), 105-121.

Marin, G., Sabogal, F., Marin, B. V., Otero-Sabogal, R., \& Perez-Stabel, E. J. (1987). Development of a short acculturation scale for Hispanics. Hispanic Journal of Behavioral Sciences, 9, 183-205.

McAuley, E., Duncan, T., \& Tammen, V. (1989). Psychometric properties of the intrinsic motivation inventory in a competitive sport setting: a confirmatory factor analysis, Research Quarterly for Exercise and Sport, 60, pp. 48-58.

McPherson, B. D. (1986). Socialization theory and research: Toward a "new wave" of scholarly inquiry in a sport context. In C. R. Rees \& A. W. Miracle (Eds.), Sport and social theory, (pp.111-134). Champaign, IL: Human Kinetics.

McGowan, W. R. (1987). Unpublished data from the University of Mississippi, University.

Mendenhall, M. E., \& Oddou, G. R. (1985). The dimensions of expatriate acculturation: A review. Academy of Management Review, 10, 39-47.

Meyer, B. (1990). From idealism to actualization: The academic performance of female collegiate athletes. Sociology of Sport Journal, 7, 44-57.

Miller, I. (2005, March 28). International student-athlete background searches: Amateur or pro? The Collegiate Sports Report. Retrieved on December 2, 2005 from http://www.nacwaa.org/rc/rc_articlepr_intlstudentathlete.php.

NCAA. (1996). 1996 NCAA study of international student-athletes. Indianapolis, IN: NCAA.

NCAA. (2004). CHAMPS/Life Skills Program. Indianapolis, IN: NCAA. 
NCAA. (2006a). NCAA graduation-rates reports. Indianapolis, IN: NCAA

NCAA. (2006b). 1999-00 - 2004-05 NCAA student-athlete race \& ethnicity report. Indianpolis, IN: NCAA.

NCAA Executive Committee. (2004, April). NCAA Strategic Plan. Indianapolis, IN: NCAA.

Newton, M., Duda, J., \& Yin, Z. (2000). Examination of the psychometric properties of the perceived motivational climate in sport questionnaire-2 in a sample of female athletes, Journal of Sport Sciences, 18, 275-290.

Nicholls, J. G. (1989). The competitive ethos and democratic education. Cambridge, MA: Harvard University Press.

Nicholls, J. G., Patashnick, M., \& Nolen, S. B. (1985). Adolescents' theories of education. Journal Educational Psychology, 77, 683-692.

Nunally, J. C., \& Bernstein, I. H. (1994). Psychometric theory ( ${ }^{\text {rd }}$ ed.). New York: McGraw-Hill.

Nuttin, J. (1980). Motivation et perspectives d'avenir. Leuven, Belgium: Presses Universitaires de Louvain.

Oberg, K. (1960). Cultural shock: Adjustment to new cultural environments. Practical Anthropology, 7, 177-182.

Palm, J. (1991). Sport for all: Approaches from utopia to reality, Schorndorf, Germany: Hofmann.

Parr, G., Bradley, L., \& Revathi, B. (1992). Concerns and feelings of international students. Journal of College Student Development, 33, 20-25.

Pascarella, E. T., Edison, M., Nora A., Hagedorn L. S., \& Terenzini, P. T. (1996). Influences on students' openness to diversity and challenge in the first year of college. Journal of Higher Education, 67(2), 174-195.

Pascarella, E. T., \& Smart, J. C. (1991). Impact of intercollegiate athletic participation for African American and Caucasian men: Some further evidence. Journal of College Student Development, 32, 123-130.

Pascarella, E. T., Truckenmiller, R., Nora, A., Terenzini, P. T., Edison, M., \& Hagedorn L. S. (1999). Cognitive impacts of intercollegiate athletic participation: Some further evidence. Journal of Higher Education, 70, 1-26. 
Pearson, R. E., \& Petitpas, A. J. (1990). Transitions of athletes: Developmental and preventive perspectives. Journal of Counseling and Development, 69, 7-10.

Phalet, K., \& Hagendoorn, L. (1996). Personal adjustment to acculturative transitions: the Turkish experience. International Journal of Psychology, 31, 131-144.

Popp, N. (2006, September). International student-athlete adjustment to U.S. universities: Testing the Ridinger and Pastore adjustment model. Paper presented at the $14^{\text {th }}$ European Association of Sport Management Congress, Nicosia, Cypress.

Rajapaksa, S., \& Dundes, L. (2002). It's a long way home: International student adjustment to living in the United States. College Student Retention, 4, 15-28.

Ridinger, L. (1998). Acculturation antecedents and outcomes associated with international and domestic student-athlete adjustment to college. Unpublished doctoral thesis, The Ohio State University, Columbus, Ohio.

Ridinger, L., \& Pastore, D. (2000a). International student-athlete adjustment to college: A preliminary analysis. NACADA Journal, 20(1), 33-41.

Ridinger, L. \& Pastore, D. (2000b). A proposed framework to identify factors associated with international student-athlete adjustment to college. International Journal of Sport Management, 1(1), 4-24.

Ridinger, L., \& Pastore, D. (2001). Coaches perceptions of recruiting international student-athletes. International Council for Health, Physical Education, Recreation, Sport, and Dance Journal, 37, 18-25.

Riemer, B. A., Beal, B., \& Schroeder, P. (2000). The influences of peer and university culture on female student athletes' perceptions of career termination, professionalization, and social isolation. Journal of Sport Behavior, 23, 364-378.

Roberts, K. (1996). Young people, schools, sport and government policies. Sport, Education, and Society, 1, 47-57.

Roseman, C. (1971). Migration as a special and temporal process. Annals of the Association of American Geographers, 61(4), 589-598.

Rosenberg, S., \& Gara, M. A. (1985). The multiplicity of personal identity. In P. Shaver (Ed.), Review of personality and social psychology (Vol. 6, pp. 87-113). Newbury Park, CA: Sage.

Rubingh, B., \& Broeke, A. (1998). The European sport club system. In L. P. Masteralexis, C. A. Barr, \& M. A Hums (Eds.), Principles and practice of sport management (pp. 195-207). Gaithersburg, MD: Aspen Publishers. 
Ryan, F. J., (1989). Participation in intercollegiate athletics: Affective outcomes. Journal of College Student Development, 30, 122-128.

Ryska, T. (2001) The impact of acculturation on sport motivation among MexicanAmerican adolescent athletes. The Psychological Record, 51, 533-547.

Salant, P., \& Dillman, D. A. (1994). How to conduct your own survey. New York, NY: John Wiley \& Sons.

Sam, M., \& Jackson, S. (2004). Sport policy development in New Zealand, International Review for the Sociology of Sport, 39(2), 205-222.

Schlossberg, N. K. (1981). A model for analyzing human adaptation to transition. The Counseling Psychologist, 9(2), 2-18.

Shavelson, R. J. (1996). Statistical reasoning for the behavioral sciences $\left(3^{\text {rd }}\right.$ ed.). Needham Heights, MA: Allyn \& Bacon.

Shin, H., \& Abell, N. (1999). The homesickness and contentment scale: Developing a culturally sensitive measure of adjustment for Asians. Research of Social Work Practice, 9, 45-60.

Slack, T., Berrett, T., \& Mistry, K. (1994). Rational planning systems as a source of organizational conflict. International Review for the Sociology of Sport, 29(3), 317-327.

Spicker, P. (2006). An introduction to social policy, Aberdeen, Scotland: The Robert Gordon University, Retrieved on June 8, 2006 from http://www2.rgu.ac.uk/publicpolicy/Introduction/wstate.htm.

Stahl, T., Rutten, A., Nutbeam, D., \& Kannas, L. (2002). The importance of policy orientation and environment on physical activity participation - a comparative analysis between Eastern Germany, Western Germany, and Finland. Health Promotion International, 17(3), 235-246.

Stahl, T., Rutten, A.., Nutbeam, D., Bauman, A., Kannas. L., Abel, T., et al. (2001). The importance of the social environment for physically active lifestyle-results from an interaction study. Social Science and Medicine, 52, 1-10.

Stevenson, C. (1990). The athletic career: Some contingencies of sport specialization. Journal of Sport Behavior, 13, 103-109.

Stidwill, H.F. (1984). Motives toward track and field competition of foreign and domestic grant-in-aid student-athletes in NCAA Division I colleges and universities. Unpublished doctoral dissertation, Oregon State University, Eugene. 
Siedentop, D. (1995). Junior sport and the evolution of sport cultures, keynote presentation to the Junior Sport Forum, Auckland, New Zealand, November.

Surdam, J., \& Collins, J. (1984). Adaptation of international students: A cause for concern. Journal of College Student Personnel, 240-245.

Tabachnick, B. G. \& Fidell, L. S. (2001). Using multivariate statistics (4 $^{\text {th }}$ ed.). Needham Heights, MA: Allyn \& Bacon.

Thorkildsen, T. A. (1988). Theories of education among academically able adolescents. Contemporary Educational Psychology, 13, 323-330.

Treasure, D. C., Carpenter, P. J., \& Power, K. T. D. (2000). Relationship between achievement goal orientations and the perceived purposes of playing rugby union for professional and amateur players. Journal of Sport Sciences, 18, 571-577.

Tyler, F. B., Brome, D. R. \& Williams, J. E. (1991). Ethnic validity, ecology, and psychotherapy. New York: Plenum Press.

Ulseth, A. (2004). Social integration in modern sport: Commercial fitness centres and voluntary sports clubs. European Sport Management Quarterly, 4, 95-115.

Vanreusel, B., Renson, R., Beunen, G., Claessens, A. L., Lefvre, J., Lysens, R., \& Vanden Eynde, B. (1997). A longitudinal study of youth sport participation and adherence to sport in adulthood. International Review for the Sociology of Sport, 32(4), 373-388.

Vogt, W. P. (1999). Dictionary of statistics \& methodology: A nontechnical guide for the social sciences $\left(2^{\text {nd }}\right.$ ed.). Thousand Oaks, CA: Sage Publications.

Weintraub, J. K., Carver, C. S., \& Scheier, M. F. (1986). Development of a theoretically based measure of coping styles. Paper presented at the annual meeting of the American Psychological Association, Washington, DC.

Westwood, M. J., \& Barker, M. (1990). Academic achievement and social adaptation among international students: A comparison groups study of the peer-pairing program. International Journal of Intercultural Relations, 14, 251-263.

White, S. A. (1995). The perceived purpose of sport among male and female intercollegiate and recreational sport participants. International Journal of Sport Psychology, 26, 490-502.

Wicker, A. W. (1979). An introduction to ecological psychology. Monterey, CA: Brooks/Cole. 
APPENDICIES 


\section{APPENDIX A}

February 15, 2007

Dear CHAMPS/Life Skills Coordinator,

My name is Nels Popp and I am a doctoral student in the Sports Administration program at the University of Louisville. I am currently working on a research project which examines the experiences of international student-athletes at NCAA Division I institutions and I need your help. Your assistance will not require much time or effort.

Here is what you will need to do:

1. Distribute surveys to selected international and domestic student-athletes at your school.

2. Encourage selected student-athletes to complete and return the surveys to you.

3. Collect all completed surveys and mail them back to me.

I will give you all materials, including the list of student-athletes chosen at your school. Surveys should not take longer than 20 minutes to complete. Distribution and collection should not last more than three weeks. I will be glad to share the results of my study with you once I am finished.

Please send me a return e-mail with your name, office phone number, and school so that I can contact you with further details regarding this important research project. If you have any questions, you can contact me at nkpopp01@louisville.edu or via phone at 502762-8497 (cell) or 502-452-8078 (office).

Thank you so much for your time and consideration.

Sincerely,

Nels Popp

University of Louisville 


\title{
APPENDIX B
}

\section{Student Adaptation to College and Purpose of Collegiate Sport Questionnaire}

Please provide answers to the information listed to the right.

\author{
1. Please circle your gender: \\ Male \\ Female \\ 2. What do you consider to be your native country? \\ 3. How many years have you been living in the United States? \\ 4. What is your approximate cumulative grade point average? \\ 5. What sport team are you a member of in college?
}

The first 27 items on this questionnaire are statements that describe college experiences. Read each one and decide how well it applies to you at the present time (within the past few days). For each item, circle the mumber at the point in contimuum that best represents how closely the statement applies to you. Circle only one mumber for each item. To change an answer, draw an " $X$ " through the incorrect response and circle the desired response.

\begin{tabular}{|c|c|c|c|c|c|c|c|c|}
\hline \multirow[b]{2}{*}{ 1. I feel that I fit in well as part of the college environment. . } & \multicolumn{3}{|c|}{ Doesn't apply to me at all. } & \multicolumn{5}{|c|}{ Applies very closely to me. } \\
\hline & 2 & 3 & 4 & 5 & 6 & 7 & 8 & 9 \\
\hline 2. I am meeting as many people and making as many friends as I would like at college. ... & 2 & 3 & 4 & 5 & 6 & 7 & 8 & 9 \\
\hline 3. I am very involved with social activities in college... & 2 & 3 & 4 & 5 & 6 & 7 & 8 & 9 \\
\hline  & 2 & 3 & 4 & 5 & 6 & 7 & 8 & 9 \\
\hline 5. I have had informal, personal contacts with college professors. . & 2 & 3 & 4 & 5 & 6 & 7 & 8 & 9 \\
\hline 6. I am pleased now about my decision to attend this college in particular. ............. & 2 & 3 & 4 & 5 & 6 & 7 & 8 & 9 \\
\hline 7. I have several close social ties at college. & 2 & 3 & 4 & 5 & 6 & 7 & 8 & 9 \\
\hline 8. Lonesomeness for home is a source of difficulty for me now. & 2 & 3 & 4 & 5 & 6 & 7 & 8 & 9 \\
\hline $\begin{array}{l}\text { 9. I enjoy living in a college dormitory. (Omit this item if you do not live in a } \\
\text { dormitory; any university housing should be regarded as a dormitory.) }\end{array}$ & 2 & 3 & 4 & 5 & 6 & 7 & 8 & 9 \\
\hline 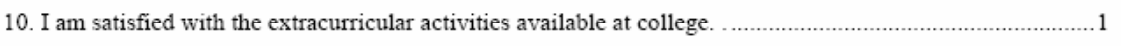 & 2 & 3 & 4 & 5 & 6 & 7 & 8 & 9 \\
\hline 11. I am getting along very well with my roommate(s) at college. (Omit if you don't have a roommate.) .........1 & 2 & 3 & 4 & 5 & 6 & 7 & 8 & 9 \\
\hline 12. I feel that I have enough social skills to get along well in the college setting. & 2 & 3 & 4 & 5 & 6 & 7 & 8 & 9 \\
\hline 13. I am having difficulty feeling at ease with other people at college. ................ & 2 & 3 & 4 & 5 & 6 & 7 & 8 & 9 \\
\hline 14. I am satisfied with the extent to which I am participating in social activities at college. . & 2 & 3 & 4 & 5 & 6 & 7 & 8 & 9 \\
\hline 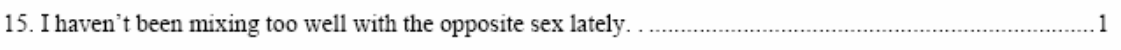 & 2 & 3 & 4 & 5 & 6 & 7 & 8 & 9 \\
\hline 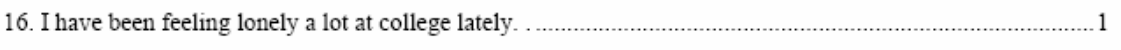 & 2 & 3 & 4 & 5 & 6 & 7 & 8 & 9 \\
\hline 17. I feel I am very different from other students at college in ways that I don't like. ....... & 2 & 3 & 4 & 5 & 6 & 7 & 8 & 9 \\
\hline 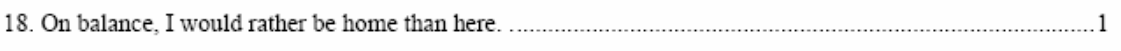 & 2 & 3 & 4 & 5 & 6 & 7 & 8 & 9 \\
\hline 19. I have some good friends or acquaintances at college with whom I can discuss any problems I may have. . 1 & 2 & 3 & 4 & 5 & 6 & 7 & 8 & 9 \\
\hline 20. I am quite satisfied with my social life at college. ............... & 2 & 3 & 4 & 5 & 6 & 7 & 8 & 9 \\
\hline 21. I am pleased now about my decision to go to college. . & 2 & 3 & 4 & 5 & 6 & 7 & 8 & 9 \\
\hline 22. I wish I were at another college or university. ................... & 2 & 3 & 4 & 5 & 6 & 7 & 8 & 9 \\
\hline 23. I am satisfied with the number and variety of courses available at college. & 2 & 3 & 4 & 5 & 6 & 7 & 8 & 9 \\
\hline  & 2 & 3 & 4 & 5 & 6 & 7 & 8 & 9 \\
\hline 25. Lately I have been giving a lot of thought to transferring to another college. & 2 & 3 & 4 & 5 & 6 & 7 & 8 & 9 \\
\hline 26. Lately I have been giving a lot of thought to dropping out of college altogether and for good. .................1 & 2 & 3 & 4 & 5 & 6 & 7 & 8 & 9 \\
\hline 27. I find myself giving considerable thought to taking time off from college and finishing later & 2 & 3 & 4 & 5 & 6 & 7 & 8 & 9 \\
\hline
\end{tabular}

Please turn this questionnaire over and complete the items on the backside. 
The 28 items listed below are different ways to complete the statement "A very important thing college sports should do is ..." Read each item and decide how much you agree or disagree with the statement. For each item, circle the mumber on the contimum that best represents how you feel. Circle only one number. To correct a response, draw an " $X$ " through the incorrect response and circle the desired response.

\begin{tabular}{|c|c|c|c|c|}
\hline A very important thing college sports should do is... & & & & $\begin{array}{l}\text { trongly } \\
\text { Agree }\end{array}$ \\
\hline 1. Teach us to be satisfied when we tried our best.. & 2 & 3 & 4 & 5 \\
\hline 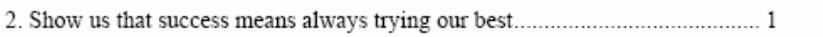 & 2 & 3 & 4 & 5 \\
\hline 3. Teach us how to exercise & 2 & 3 & 4 & 5 \\
\hline 4. Keep people fit & 2 & 3 & 4 & 5 \\
\hline 5. Show us how we can be physically active all our lives................................. 1 & 2 & 3 & 4 & 5 \\
\hline 6. Teach us to sacrifice pleasure and work to do the right thing & 2 & 3 & 4 & 5 \\
\hline 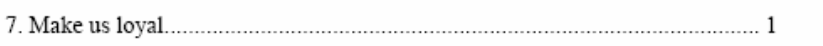 & 2 & 3 & 4 & 5 \\
\hline 8. Prepare us for jobs that will allow us to help others .................................. 1 & 2 & 3 & 4 & 5 \\
\hline 9. Teach us to respect authority & 2 & 3 & 4 & 5 \\
\hline 10. Prepare us to do things we have to do, even if we don't want to $\ldots \ldots \ldots \ldots \ldots \ldots . . . . .1$ & 2 & 3 & 4 & 5 \\
\hline 11. Make us responsible law-abiding citizens & 2 & 3 & 4 & 5 \\
\hline 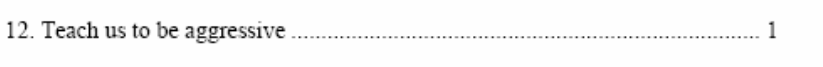 & 2 & 3 & 4 & 5 \\
\hline 13. Make us mentally tough & 2 & 3 & 4 & 5 \\
\hline 14. Teach us the "killer instinct" & 2 & 3 & 4 & 5 \\
\hline 15. Teach us to do what is necessary to be the best around ................................ 1 & 2 & 3 & 4 & 5 \\
\hline 16. Give us the chance to get a college education & 2 & 3 & 4 & 5 \\
\hline 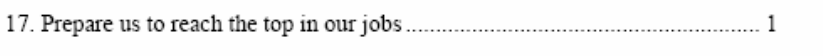 & 2 & 3 & 4 & 5 \\
\hline 18. Help us move into a job which pays good money ................................... 1 & 2 & 3 & 4 & 5 \\
\hline 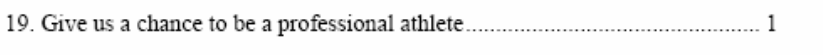 & 2 & 3 & 4 & 5 \\
\hline 20. Make us feel important & 2 & 3 & 4 & 5 \\
\hline 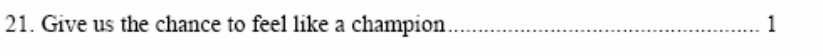 & 2 & 3 & 4 & 5 \\
\hline 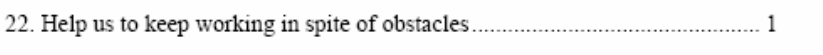 & 2 & 3 & 4 & 5 \\
\hline 23. Teach us to set high standards for our own work... & 2 & 3 & 4 & 5 \\
\hline 24. Give us self-confidence & 2 & 3 & 4 & 5 \\
\hline 25 . Weed out those who don't have what it takes...... & 2 & 3 & 4 & 5 \\
\hline 26. Help us be popular among our friends & 2 & 3 & 4 & 5 \\
\hline 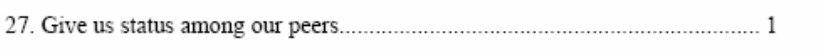 & 2 & 3 & 4 & 5 \\
\hline 28. Show us how to be better than most people & 2 & 3 & 4 & 5 \\
\hline
\end{tabular}




\section{APPENDIX C}

April 13, 2007

Dear

A few days ago, I spoke to you regarding research we are conducting at the University of Louisville examining differences in adjustment to college between international and domestic student-athletes. The issue of international student-athlete welfare is a vastly under-researched area and it is hoped this study will open the door to important and intriguing findings regarding the student-athletes with which you work so hard to assist.

Thank you so much for your willingness to assist with this research project.

Enclosed are several surveys. Attached to each survey are a name tag, an instruction sheet, and an envelope. Please distribute these surveys to the student-athletes listed on the name tags. You may choose whichever distribution method you believe will be most effective, whether it is hand delivered, sent through campus mail, or distributed via team personnel.

After completing the survey, student-athletes are instructed to seal the survey in the envelope and return it to you, preferably through campus mail as to protect anonymity.

Also enclosed is a list of all student-athletes selected at your school. In approximately one week, I will send you an e-mail in which I will thank student-athletes for completing the survey and ask those who have not completed the survey to please try to do so. This e-mail will also indicate that extra surveys are available in your office. Please forward this note via e-mail to all student-athletes on the list.

After approximately two weeks, I will ask you to return all completed surveys to me. Enclosed is a package with the postage already paid.

I would like to sincerely thank you again for your assistance in this data collection. I know you are quite busy and I am very grateful that you have taken time out of your schedule to help with this project.

If you have any questions or would like to know the results of our study, please feel free to e-mail me at nkpopp01@louisville.edu or call me at 502-452-8078 (office) or 502-7628497 (cell).

Sincerely, Nels Popp 
APPENDIX D

\section{International Student-Athlete Perception of College Sport and its Effect on Adjustment to College}

March 19, 2007

Dear Student-Athlete:

You are being invited to participate in a research study by answering the attached survey about adjustment to college and perception of the purpose of sport for international and domestic student-athletes. The information collected will be very valuable to the NCAA and its administrators, coaches, and student-athletes. There are no known risks for your participation in this research study. The information collected may or may not benefit you directly. The information learned in this study may be helpful to others. The information you provide will be compared to results from other international and domestic student-athletes to determine if there are differences in how student-athletes adjust to college. Recommendations based on the results will be shared to help improve the adjustment process for future student-athletes. Your anonymous, completed survey will be stored at the University of Louisville. The survey will take approximately 10 minutes to complete. When finished, please seal your survey in the enclosed envelope and return it to your school's CHAMPS/Life Skills coordinator.

Individuals from the University of Louisville's Department of Sports Administration, the Institutional Review Board (IRB), the Human Subjects Protection Program Office (HSPPO), and other regulatory agencies may inspect these records. In all other respects, however, the data will be held in confidence to the extent permitted by law. Should the data be published, your identity will not be disclosed.

Taking part in this study is voluntary. By completing this survey you agree to take part in this research study. You do not have to answer any questions that make you uncomfortable. You may choose not to take part at all. If you decide to be in this study you may stop taking part at any time.

If you have any questions, concerns, or complaints about the research study, please contact researcher Nels Popp at (502) 762-8497 or Dr. Mary Hums at (502) 852-5908.

If you have any questions about your rights as a research subject, you may call the Human Subjects Protection Program Office at (502) 852-5188. You can discuss any questions about your rights as a research subject, in private, with a member of the Institutional Review Board (IRB). You may also call this number if you have other questions about the research, and you cannot reach the research staff, or want to talk to someone else. The IRB is an independent committee made up of people from the 
University community, staff of the institutions, as well as people from the community not connected with these institutions. The IRB has reviewed this research study.

If you have concerns or complaints about the research or research staff and you do not wish to give your name, you may call 1-877-852-1167. This is a 24 hour hot line answered by people who do not work at the University of Louisville.

Sincerely,

Nels Popp

Doctoral Candidate

University of Louisville

Mary A. Hums PhD

Professor

University of Louisville 


\section{APPENDIX E}

$\mathrm{Hi}$

Thank you so much for your help with my research project. I hope this is not adding too much to your plate.

If you feel it would help in your survey collection, there is a friendly reminder below reminding student-athletes to turn in their surveys. If you can forward this via e-mail to your student-athlete list, that would be great.

I have attached your student-athlete list and a copy of the survey, if you need more.

Please let me know if you are having any difficulties. I will call you later next week to see if you are ready to send back responses.

Again, thank you so much for your help with this project.

Sincerely,

Nels Popp

Dear Student-Athlete,

Recently, you were given a survey regarding adjustment to college and the purpose of sport. The information collected from this survey will provide important details for researchers examining college athletics today.

If you have already completed and returned this survey, thank you very much for your time. We sincerely appreciate your effort.

If you have not yet completed this survey, we ask that you please consider doing so at your earliest convenience. If you no longer have your copy of the survey, you can receive an additional copy from in the athletics office.

Thank you so much for your participation with this important research.

Sincerely,

Nels Popp

Doctoral Candidate

University of Louisville 


\section{CURRICULUM VITAE NELS POPP}

Home:

1216 Lipps Lane \#104

Louisville, KY 40219

(502) 762-8497

nkpopp01@louisville.edu
Office:

Bellarmine University/Knights Hall

Department of Athletics

Louisville, KY 40205

(502) 452-8078

npopp@bellarmine.edu

\section{EDUCATION}

PhD, Educational Leadership and Organizational Development - Sport Administration, University of Louisville, August 2007

Dissertation title: International student-athlete perception of college sport and its effect on adjustment to college. Chairpersons: Mary A. Hums PhD and T. Christopher

Greenwell PhD

MS, Exercise and Sport Science - Sport Administration, University of Wisconsin - La Crosse, August 2004

BA, English Literature, Saint Mary’s University (Minn.), May 1997

\section{WORK EXPERIENCE}

\section{Academic Positions Held}

Adjunct Professor, Bellarmine University, 2007 - present

-Taught undergraduate course in Sports and the Media

Adjunct Professor, University of Louisville, 2005-2006

-Taught undergraduate course in Aspects of Sport Law

-Taught undergraduate and graduate courses of Sports Facility Management

Co-taught undergraduate and graduate course in International Sport 


\section{Other Professional Employment}

Sports Information Director, Bellarmine University (Louisville, Ky.), 2004 - present

Sports Information Director, Viterbo University (La Crosse, Wis.), 2002-2004

Sports Information Director, Midwest Classic Conference (La Crosse, Wis.), 2000-2004

Assistant Men’s Basketball Coach, Viterbo University (La Crosse, Wis.), 2000-2004

Media and Promotions Officer, Cairns Taipans (Cairns, Australia), 1999-2000

Administrator and Media Officer, Cairns Basketball Inc. (Cairns, Australia), 1997-1999

\section{RESEARCH}

\section{$\underline{\text { Articles }}$}

Greenwell, T. C., Jordan, J. S., Popp, N., \& Brownlee, E. (in press). Customer preference and student tickets: Using conjoint analysis to develop ticket policy. International Journal of Sport Management.

Greenwell, T. C., Jordan, J. S., Brownlee, E., \& Popp, N. (in review). Service fairness in spectator sport: The importance of voice and choice on customer satisfaction. Submitted to Sport Marketing Quarterly.

Popp, N., Hums, M. A., \& Keedy, J. L. (manuscript in preparation). International student-athlete adjustment to U.S. universities: Testing the Ridinger and Pastore model. Manuscript to be submitted to the International Journal of Sport Management.

\section{$\underline{\text { Presentations at refereed conferences }}$}

Popp, N., Hums, M.A., \& Greenwell, T.C. (2007, June). Perceived purpose of collegiate sport and its effect on social adjustment and institutional attachment: A comparison of international and domestic NCAA student-athletes. Presented at the meeting of the North American Society for Sport Management, Fort Lauderdale, Fla.

Popp, N., Hums, M.A., \& Greenwell, T.C. (2007, June). Do international studentathletes view collegiate sport differently than domestic student-athletes? Presented at the meeting of the North American Society for Sport Management, Fort Lauderdale, Fla.

Popp, N. (2007, March). Do international student-athletes adjust better to college? Presented at The Drake Group Conference. Cleveland, Ohio. 
Popp, N. (2006, September). International student-athlete adjustment to U.S. universities: Testing the Ridinger and Pastore model. Presented at the meeting of the European Association for Sport Management $14^{\text {th }}$ Annual Congress, Nicosia, Cyprus.

Greenwell, T. C., \& Popp, N. (2006, June). Customer preference and student tickets: Using conjoint analysis to develop ticket policy. Presented at the meeting of the North American Society for Sport Management, Kansas City, Mo.

Greenwell, T. C., Jordan, J. S., \& Popp, N. (2006, June). The effects of voice and choice on customer satisfaction with student ticketing policy. Presented at the meeting of the North American Society for Sport Management, Kansas City, Mo.

\section{GRANTS}

Popp, N. (2007, May). NCAA Student Research Grant. Submitted to the National Collegiate Athletic Association in the amount of $\$ 5,000$. (pending)

Popp, N. (2007, March). Travel Grant. Submitted to the University of Louisville's Department of Health and Sport Sciences Student Research Committee in the amount of \$175. (funded)

Popp, N. (2006, August). NCAA Student Research Grant. Submitted to the National Collegiate Athletic Association in the amount of \$5,000. (unfunded)

Popp, N. (2006, July). Travel Grant. Submitted to the University of Louisville Graduate Student Council in the amount of $\$ 300$. (funded)

Popp, N. (2006, June). Travel Grant. Submitted to the University of Louisville International Student Center in the amount of $\$ 500$. (funded)

Popp, N. (2006, May). CoSIDA Graduate Internship Grant. Submitted to College Sports Information Directors Association for academic year 2006-07 in the amount of \$10,000 (funded)

Popp, N. (2006, May). Travel Grant. Submitted to the University of Louisville Graduate Student Council in the amount of $\$ 250$. (funded)

Popp, N. (2005, May). CoSIDA Graduate Internship Grant. Submitted to College Sports Information Directors Association for academic year 2005-06 in the amount of \$10,000 (unfunded)

Popp, N. (2004, March). Travel Grant. Submitted to the University of Wisconsin-La Crosse Graduate Student Council in the amount of \$200. (funded) 


\section{TEACHING}

\section{Graduate and Undergraduate Courses}

Sports and the Media (Bellarmine University)

Aspects of Sport Law (University of Louisville)

Sports Facilities Management (University of Louisville)

International Sport - Co-taught with Dr. Mary Hums (University of Louisville)

\section{$\underline{\text { Guest Course Lectures }}$}

“The College Athletics Business Model” (2007, July). Economics of Sport - MBA Course, Bellarmine University. Course instructor Dr. David Brown.

"Social Positioning and the Construction of a Youth Sports Club” (2006, April). Problem Analysis in Educational Administration and Leadership. University of Louisville. Mentored by Dr. Paul Winter.

"NCAA and Foreign Student-Athlete Eligibility” (2005, August). NCAA Compliance and Legal Issues, University of Louisville. Mentored by Professor Anita Moorman.

“College Baseball and Summer Leagues” (2005, May). The Business of Baseball, University of Louisville. Mentored by Dr. Mary Hums.

\section{SERVICE}

\section{$\underline{\text { Professional Memberships }}$}

North American Society for Sport Management (NASSM), 2005-present

- NASSM Student Board Representative, 2006-2007

College Sports Information Directors Association (CoSIDA), 2004-present - Membership Recruitment Committee, 2006-present

$\underline{\text { National and International Conferences Attended }}$

North American Society for Sport Management (NASSM) 2007 Fort Lauderdale, Fla.

The Drake Group Annual Conference 2007 Cleveland, Ohio

European Association for Sport Management (EASM) 2006 Nicosia, Cyprus 
North American Society for Sport Management (NASSM) 2006 Kansas City, Mo. College Sports Information Directors Association (CoSIDA) 2006 Nashville, Tenn. North American Society for Sport Management (NASSM) 2005 Regina, Canada College Sports Information Directors Association (CoSIDA) 2005 Philadelphia, Pa. National Association of Intercollegiate Athletics (NAIA) 2004 Kansas City, Mo.

\section{Related Relevant Experience}

Staff Writer, Geezer Jock Magazine - Daily Newspaper Coverage of National Senior Games (Louisville, Ky.), 2007

Event Staff, AVP 2007 Louisville Beach Volleyball Open (Louisville, Ky.), May 2007

Game Day Operations Intern, La Crosse Loggers - Northwoods College Summer Baseball League (La Crosse, Wis.), 2004

Basketball camp coach, University of Wisconsin; University of Minnesota; Wright State University; UW-Green Bay; UW-Stevens Point; Viterbo University; \& Cairns Basketball Inc. (various locations), 1997-2004

Varsity basketball player, Saint Mary’s University (Winona, Minn.), 1994-1997

Intramural student director, Saint Mary’s University (Winona, Minn.), 1995-1997

\section{Volunteer Work}

Panel Moderator, NASSM Student Board Panel (Fort Lauderdale, Fla.), June 2007

Event Volunteer/Scoreboard Operator, Derby Music Jam (Louisville, Ky.), May, 2006

Emcee, Bellarmine Athletics Department Golf Outing Dinner (Elizabeth, Ind.), May, 2006

Emcee, Bellarmine Knight of Knights Dinner (Louisville, Ky.), October, 2005

Event Volunteer, American Cancer Society/Midwest Securities Run/Walk (La Crosse, Wis.), April, 2004

Coach, Cairns Under-18 boy’s representative basketball team (Cairns, Australia), 1999 Staff Writer, The Cardinal Saint Mary’s student newspaper (Winona, Minn.) 1995-97 
Intern/TV Reporter, Sport Department Channel 7 (Cairns, Australia), 1995

$\underline{\text { Honors }}$

“Good Knight” Award, Bellarmine Athletics Department (Louisville, Ky.) 2005

Student Service Award, Saint Mary’s University (Winona, Minn.) 1997 